\title{
Parametric architectural design solutions - some observed difficulties of application
}

\author{
Matthew Fraser \\ A thesis submitted to the Victoria University of Wellington \\ in fulfilment of the requirements for the degree of Master of Architecture
}

Victoria University Wellington 
Acknowledgements:

Michael Donn as (tolerant and enduring) supervisor

Jeni Mihova for providing inspiration to explore Greek architecture digitally

Special thanks to the co-ordinators of ARCI212; Martin Hanley and Guy Marriage; as well as tutor comments and observations for ARCI212 and SARC223 courses;

Special thanks also to the authors of highlighted student work in section two; Monique Mackenzie, Alex Sawicka-Ritchie. 


\begin{abstract}
It is generally accepted that the restrictions of digital parametric design tools (DPDT's) are currently shifting. Technology and interface can be engineered to overcome many complex problems within architectural processes, yet this does not necessarily mean solutions are easier to obtain; quite the opposite can occur. As architectural practitioners lean more towards the ubiquitous use of digital processes the decisions made by the designer can be overshadowed by the advantages of time saving technologies and heuristic rules of thumb. The various inherent interfaces of digital processes tend to conceal the real complexity and nuance of the set of possible solutions and analyses.
\end{abstract}

The awareness of the workings of the tools and processes from a 'base principle' point of view could be a weak point in regards to the uptake by a new generation of architects who experience a growing simplification of interfaces within new digital design processes. This poses a problem for a profession which seeks to integrate technical skills knowledge into wider, sometimes loosely structured, set of processes. This research highlights the problems faced by architects, students, and specialists who aspire to apply innovative digital processes in a strategic manner. The concern which arises is this lack of understanding of how tools could be used reduces the ability to integrate them within design process. Theoretical literature falls short in providing robust guidance to the application of illunderstood design tools, further compounding the sincere integration of practical solutions.

There are ways forward for developers to create new and updated tools, such as form modellers, design aids, and optimisation helpers, but these do not necessarily meet their full potential in providing integrated solutions. It is possible to improve our understanding of these potentials by looking at how these tools can be used in both practical and theoretical terms. In this thesis this is done by framing and answering the following research question:

\title{
What are the characteristics of some of the observed issues and obstacles revealed in the practical application of digital parametric design tools in architecture?
}

This question is adopted so that the obstacles of DPDT's may be better understood and allow for better integration, better design outcomes, and better future use of powerful latent potential. This research defines the process of parametric design through analysis of literature and applies this to participantobserver case studies. It finds that a clarification of particular issues and obstacles can be useful to improved understanding of DPDT's and that certain qualitative characteristics of these obstacles can constrain applications and avenues of exploration. 


\section{Contents}
$0.1 \quad$ Abstract
0.2 Table of Figures

1.0

1.1

1.1 .1

1.1.3

1.1 .4

1.3

1.4

\section{0}

Some Obstacles in Modelling Parthenon Elements with Parametric Tools

3.0 Thinking through design processes $4 k$

3.1 .

3.2

3.3 .

3.4 .

3.5.

3.6.

3.7 .

3.8 . Foreword Introduction Background Analysis

Discussion

Conclusions

Case Study Considerations

Background of Parthenon Columns Project

Overview of Case

Source Material

Iterative Models and Problem Development

Manufacturing Considerations, Export and Checking

Research Field and Focus

Hypothesis

Selected Approaches

Document Structure 


\subsection{Table of Figures}

Figure 1. Cumulative Index of Computer Aided Architectural Design (CumInCAD) listed papers over three decades

Figure 2. Sketchpad interaction by modeller after Sutherland

Figure 3. A circle; solving dependent variables in parameterised relationships after Wolfram

Figure 4. Explicit, parametric, and associative models, after Burry (2003)

Figure 5. Cyclical architectural processes (in part after Sutherland)

Figure 6. Changes to dependencies in a parametric model, after Burry (2003)

Figure 7. Illustration of possible (and relatively superfluous) variation (Author image)

Figure 8. Possibilities of unconstrained and constrained box generation (Author image)

Figure 9. Possible design moves represented in Linkographs after Kan and Gero (2005)

Figure 10. Thesis and document structure

Figure 11. Experimentation prototypes for Educational Games project from large to small scale. (Author images also with J.Mihova)

Figure 12. Project workflow from source material to physical artefact

Figure 13. Project overview; Development of source material into multiple iterative models (Author images)

Figure 14. Parthenon anatomy after Neimann

Figure 15. Parthenon hyper refinements after Korres

Figure 16. Hyper refinements of Greek temples; (a) after Tansley and Kleiner (b) after Dismoor Column (c) after Coulton

Figure 17. Diminution method in Greek temple Stylobase after Dinsmoor

Figure 18. Stylobase setout and flexibility (Author image)

Figure 19. Column axis placement and incline and degrees of freedom (Author image)

Figure 20. Illustration of problematic individual surfaces method of modelling a column (Author image)

Figure 21. Column first iteration set (Author images)

Figure 22. Model from initial solid column (Author images) 
Figure 23. 'Unique column' method (Author images)

Figure 24. Final Column solid modelling sequence (Author images)

Figure 25. Epistylion modelling stage (Author images)

Figure 26. Integrated model (Author images)

Figure 27. Enlargement of final model (Author image)

Figure 28. Final 1:150 prototyped models (Author images)

Figure 29. Diagram of current course structures

Figure 30. Survey responses

Figure 31. Covered transit station, showing illumination levels lighting analysis overlays (Monique Mackenzie image)

Figure 32. Subterranean transit station (Alex Sawicka-Ritchie image) 


\subsection{Research Field and Focus}

Developments in digital design tools and the culture in which these are applied have in a short time changed the way architects and collaborators can look at different design problems and the characteristics of design solutions. Architects can use digital tools to assist design investigations, decision making and exploring opportunities to produce novel or innovative outcomes. Some of these tools are identified as parametric tools. Information regarding the processes of digital parametric design tools (DPDT's) and qualitative accounts of how these are incorporated into architectural problem solving are valuable to those coming into the field. Hudson (2011) suggests that research in this field can be put into two categories; theoretical and practical literature, a distinction which has similarities to the theoretical and empirical branches of the physical sciences. The distinction is made between what can be perceived as opaque theoretical texts and practical literature which despite its brevity and at times lack of scrutiny, is more helpful and relevant to understanding architectural processes. This thesis adopts the practical, or empirical, approach to the following research question:

What are the characteristics of some of the observed issues and obstacles revealed in the practical application of digital parametric design tools?

The goal of research of this type is to better understand the obstacles to the use of DPDT's and thus potentially allow for better integration, better design outcomes, and better future use of their powerful latent potential. This research defines the process of parametric design through analysis of literature, thus placing the empirical within a theoretical framework, and then applies this to participant-observer case studies.

Design computation more than other areas of research is defined more by process and tasks than by rigid definitions and theoretical models. Practical literature in this field, and the sub field of parametrics, is on the increase (fig 1.) and much of this may be found in the Cumulative Index of Computer Aided Design (CumInCAD) database ${ }^{1}$.

\footnotetext{
${ }^{1}$ The self description of CUMINCAD from http://cumincad.scix.net/; "CumInCAD is a cumulative index of publications about computer aided architectural design. It includes bibliographic information about over 10,000 records from journals and conferences such as ACADIA, ASCAAD, CAADRIA, eCAADe, SiGraDi, CAAD futures, DDSS and others. All papers include full abstracts. Full texts, in PDF, of some 7,200 papers are also available."
} 


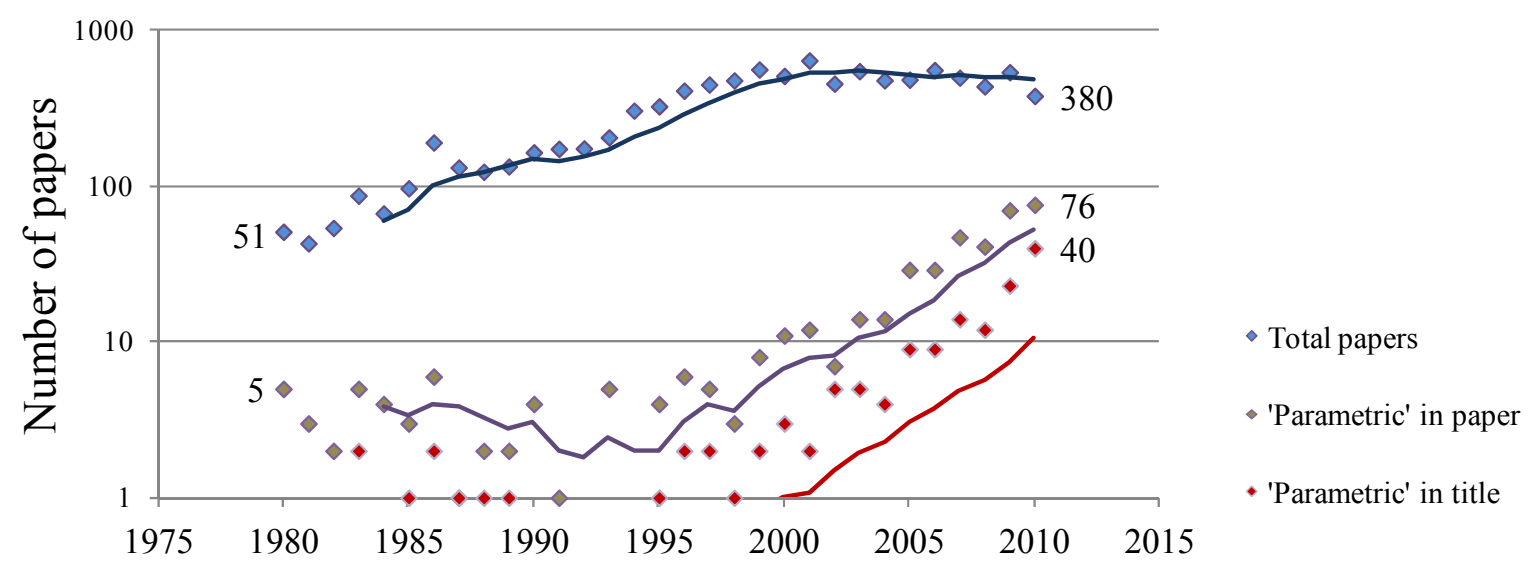

Figure 1. Cumulative Index of Computer Aided Architectural Design (CumInCAD) listed papers over three decades. Use of the term 'Parametric' in papers and titles is increasing.

Mueller (2009a, 2011) outlines three axes for the field of 'computational design', while identifying that there is continuing debate regarding the areas of research included in the field as well as the name of the field itself. These are design investigations, project lifecycle, and practice models/supporting technologies. Practice models/supporting technologies is identified by Mueller as the axis where current research is reconfiguring boundaries through developments in interoperability (ibid). This interoperability area of practice is identified as key to the future of digital architecture, and there is a developing overlap between CAAD and Design Cognition conference participants and research which addresses some of the problems brought on by the lack of clear and dominant definitions within the field. There is a growing mix of computer science and ethnographic methods in design research. One could interpret this as a sign that there are numerous ways forward, and efforts to expand research methods and field overlaps continue. Mueller (2009b) and Jabi (2007) reiterate one of the major issues in the field as lack of a clear taxonomy. Further, Bhatt et al. $(2006,2008,2009)$ identify that the track record of the field determines the domain rather than a singular dominant paradigm and undertake several studies into ways of representing the ontology of the field, mainly through analyses of peer-reviewed papers ${ }^{2}$ and listings on CumInCAD (fig1).

\footnotetext{
${ }^{2}$ Bhatt's $(2008,2009)$ work highlights both the developing state of CumINCAD in volume and cross referencing of key terms. Findings are that there is a loose overarching taxonomy, and that progress is slowly being made in unification of the field, although a broadening of the domain is a positive development.
} 
There is some research now occurring in a range of areas of human-computer interaction and cognition in design, which are beyond of the traditional boundaries of the CAAD field ${ }^{3}$. This new research is partly interested in how we might reconfigure the role of digital tools in architecture without an overarching theoretical model and dominant paradigm. It is also apparent that any potential mass uptake of DPDT's progresses with or without well accepted definitions or taxonomy. Some authors have in recent years published books which are bucking the trend and structuring chapters around semi-taxonomic organization without implying a singular theoretical structure is essential ${ }^{4}$. Literature suggests that whilst the current trend of DPDT's in architectural practice and culture is that of a widening application of available tools ${ }^{5}$ and the creation of new and easily navigable systems ${ }^{6}$, it also suffers from a lack of achieving its full potential. The mass uptake of these tools is mainly due to the aspirations of training institutions and individual firms searching to find new routes to explore the future of contemporary practice. Generally, tools used in standard practice tend to have a direct and simple goal: to assist information management within the design and documentation process. Training within architecture schools is provided as a means of both preparing students to use the tools they will likely encounter in practice and to explore directly the research implications of theories and philosophies of design in the "digital age" . These ambitions of technical education for architects and the connection to integrated design have in part stimulated the research in this thesis.

\footnotetext{
${ }^{3}$ Martens et al. (2007): "In later years the external sources [areas of research] became more pronounced, especially from areas such as artificial intelligence and cognitive science, which were characterized by similar preoccupations with human and machine intelligence." Koutamanis et al then suggest that CAAD theory between the modernist decades and the 1990's "retained its coherence".

Also, Mueller (2009b): “...our research community as encompassed by eCAADe, ACADIA, CAADRIA, SIGraDi, ASCAAD, and the CAAD Futures organizations, as well as other overlapping or related research communities like the community around the Design Computing and Cognition conference series or more generally related single-shot conferences, such an effort [development of an ontology] has also the potential to enhance the sense of a continuum of research across regions, as well as across focus areas."

${ }^{4}$ General examples include Woodbury (2010) and Burry and Burry (2010, 2011).

5 "The traditional idea of computer aided drafting and the tools needed to support it have saturated architectural offices." (Mueller et al. 2003). "Parametric modelling is becoming more standard within CAD" (Hernandez 2006). "The last decade, marked by the rapid development of widely available computational tools, concentrated mainly on the idea of parametric, and, more recently, integrated design..." (Achten et al. 2011a)

${ }^{6}$ General application: Achten (2011b), Aish (2009); Case studies Fernando et al (2010), Toth et al (2011), also push for standardised interoperability (AIA 2009)

${ }^{7}$ Cheng (2006), Friedman (2006), AIA (2006), RIBA (2011), AACA (2006) also Koutamanis (1993a), Burry (2005)
} 


\subsection{Defining the Field Of Inquiry: Parametric Divisions}

The term parametric is applied in architecture in several ways. In the broadest sense "...all design acts on an evaluation of a range of parameters during any given process" (Burry 2003 p149) is a view which can be seen as both common sense and originating in a formal analysis of design problem solving processes. The idea that design is a response to the parameters of a design 'problem' is a commonly held view and in this current use can be traced back at least as early as Alexander's analysis of design problem decomposition in Notes on the Synthesis of Form (Alexander 1964) ${ }^{8}$. One widespread way of introducing and describing what is meant by parametric follows etymological definitions of the term ${ }^{9}$. Essentially, this approach builds upon the standard definition of para-metric in Greek as 'auxiliary-measure ${ }^{10}$ which allows a set of solutions to be expressed by a set of dynamic relationships to allow variation and permutation. That design deals with these possible variations as a result of parameters within the problem solving process means that it can be viewed as parametric $^{11}$; the problem can be mapped into a parametric model through expressions of auxiliary, or algorithmic ${ }^{12}$ relationships.

Parametric equations in mathematics also come to mind and the definition can be beneficial, although not essential in understanding a more architectural definition of parametric design. From Wolfram Mathworld: "Parametric equations are a set of equations that express a set of quantities as explicit functions of a number of independent variables, known as "parameters."” (Weisstein/ Wolfram2011). The Wolfram reference illustrates this definition using equations which define a circle in Cartesian coordinates. The variables are shown to be dynamic and the equation may be arranged in different ways depending on the required solution (see fig 3). This illustration shows a circle defined by trigonometric, Pythagorean, and graphical methods. Each way of defining these relationships can be seen as a different model. All represent a very strong and simple pattern. Each model is a parametric expression of a set of instances which can occur. This definition is relevant to the architectural one in an

\footnotetext{
${ }^{8}$ Also, arrangement of abstracted problem and solution variables can be traced back even further to Euclid's Data and Elements. Euclid (300BC), Mueller, I. (1981)

${ }^{9}$ Burry 2003, Woodbury 2010, Hudson 2011

${ }^{10}$ OED (2004) Auxiliary measure. Meaning dynamic or algorithmic relationships

${ }^{11}$ Kolarevic (2003) defines 'parametrics' in design as "In parametric design, it is the parameters of a particular design which are declared, not its shape” (p.17)

${ }^{12}$ Understood as Algorithm $=$ logic + control, Kowalski (1979)
} 
algorithmic sense as it encompasses formal aspects of both compositional logic and geometric control.

Within architecture, the term computational design ${ }^{13}$ is widely used, but loosely defined, and is sometimes interchangeable with design computation. This is typical within the field. It is arguable, for example, that all digital tools are parametric as they build relationships between input variables and outputs (parameters) within a dynamic system. These relationships may be seen as parametric in nature. Relying on formal logic, the abstract objects inside the program are defined in a way that means they can update instantly (or at least somewhat quickly) along lines of dependency. A ubiquitous example of this is the development and use of spread sheet programs such as MicrosoftExcel. The development and use of object oriented programming highlights the significance of encoding dynamic relationships into reusable computing solutions. One early example of this in the Computer Aided Design (CAD) field can be found in the graphical user interface of the digital design system Sketchpad, created by Ivan Sutherland in 1960, where the notions of object and instance were defined as an expression of parametric objects and the values which can occur through constraint propagation ${ }^{14}$. Here, the objects could be modified to meet constraints specified by the user, or modeller, in situation described by fig 2 .

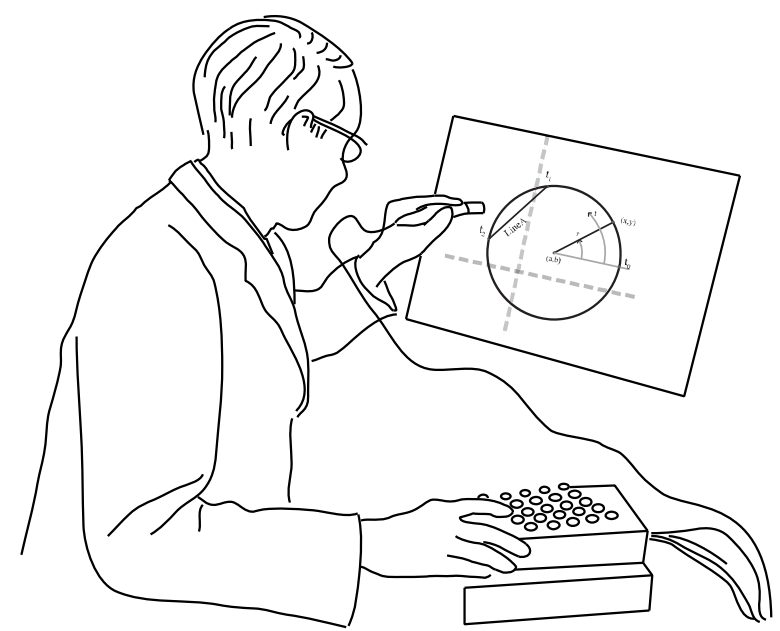

Figure 2. Sketchpad interaction by modeller after Sutherland

\footnotetext{
13 "The term "computational design" is as ill-defined as most other terms in this research domain. With increasing definition this term could turn out to be synonymous with "computer aided design" (CAD), or could be discovered to be a subcategory of CAD. Hypothetically, "computational design" could also be revealed as the super-category to "computer aided architectural design” (CAAD)." Mueller (2009b)

Further, the intended meaning in relation to design is that of computation of design rather than the design of computational systems, although both meanings are applicable to architectural 'design computation'.

${ }^{14}$ Constraint propogation by means of a 'relaxation' technique. Sutherland (1963).
} 


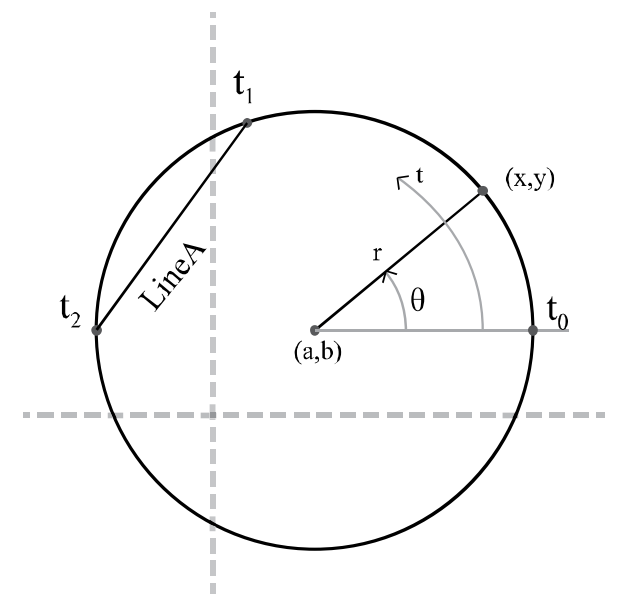

$$
\begin{gathered}
\left.x^{2}+y^{2}=r^{2} \quad \text { (when } a, b=0\right) \\
x=a+r \cos (\theta) \\
y=b+r \sin (\theta) \\
\left.x=a+r \frac{1-\theta^{2}}{1+\theta^{2}}\right)(\theta \text { in ra } \\
\left.y=b+r \frac{2 \theta}{1+\theta^{2}}\right\}\left(\begin{array}{l}
\theta<t<=1), \\
\text { (in degrees) } t
\end{array}=\frac{\theta}{360} \quad(\text { in radians }) t=\frac{\theta}{2 \pi}\right.
\end{gathered}
$$

Figure 3. A circle; solving dependent variables in parameterised relationships, after Wolfram.

The arrangement of dependent and independent variables depends on the 'problem' being solved and the 'given' quantities in the equations; trigonometric functions and an inscribed triangle.

In the Wolfram example of the parametrically defined circle, the concept of parametric rather than Cartesian geometry emerges when we consider how the circle model can be expressed as abstract objects. Elements of a model, as illustrated in Fig 3, such as LineA between points $\mathrm{t}_{1}$ and $t_{2}$ can be expressed in different ways. Thus an object like LineA can be defined not only as static values of line start point and end point coordinates in the $x$ and $y$ dimensions, but rather as reference 'placeholder' objects $\left(t_{1}\right)$ and $\left(t_{2}\right)$, which are defined by the user as separate subroutines. Thus, in the example in Fig 3, $\left(\mathrm{t}_{1}=0.3\right),\left(\mathrm{t}_{2}=0.5\right)$ are an expression of parameter values of distance along the curve of the circle. An even more 'dense' algorithmic expression could define a relationship between $t_{1}$ and $t 2$ such that defining the position of $t_{1}$ also defines $t_{2}:\left(t_{1}=0\right),\left(t_{2}=t_{1}+0.2\right)$; thus further constraining the solution and allowing a single user controlled parameter to define a constrained LineA inside the circle.

The arrangement of objects and algorithms with regard to user interaction can both constrain and enable a parametric model. Changes to these subroutines constrain the start and end points of LineA to locations on the circle in a way similar to the Burry (2003) illustration distinguishing between types of associative relational geometry; where a line between constrained points and in relation to other objects can be expressed in multiple ways (fig 4). 


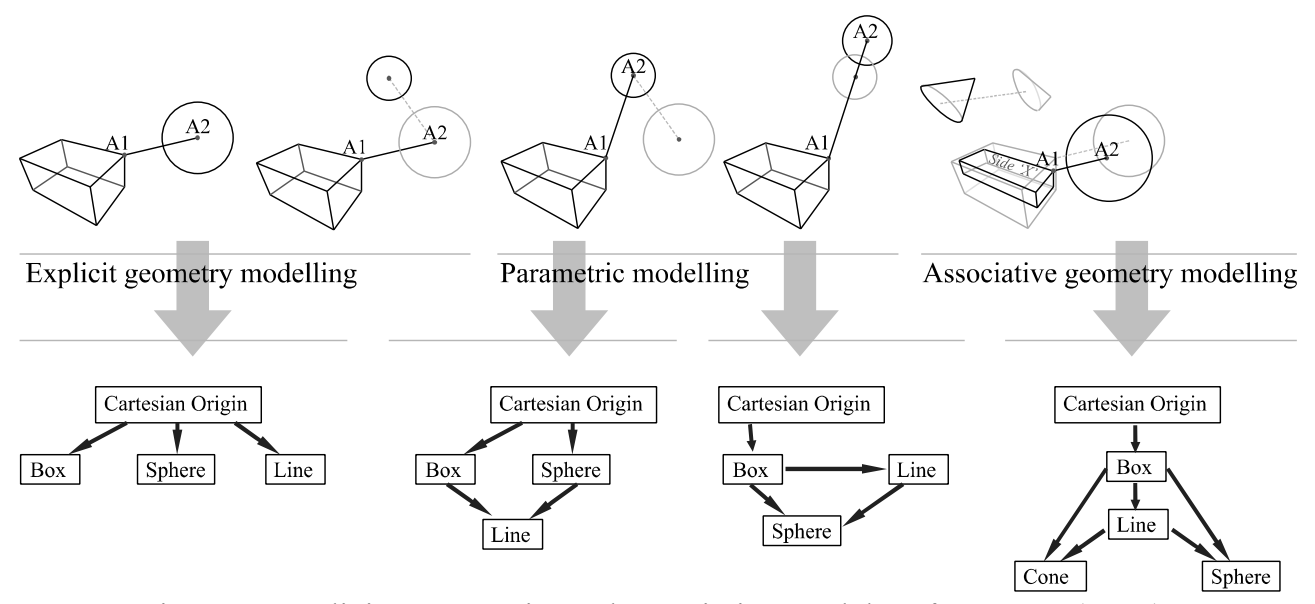

Figure 4. Explicit, parametric, and associative models, after Burry (2003); changes to the model in three cases ${ }^{15}$ and corresponding schema,

Parametric design in this thesis refers principally to the creation and modification of a parametric model by a modeller within a platform which allows associative relationships to be created and manipulated. The general application of parametric relationships within programming combined with the development of digital design tools has resulted in a range of programs which are focussed towards architectural design processes. Whilst some programs have simple interfaces which 'black-box' the internal workings and relationships between objects or elements, more complex tools allow for an application programming interface (API) or equivalent methods. The more complex tools in architectural parametric design allow for detailed manipulation of the parametric relationships thus facilitating the use of numerous methods by which elements and data sets can be associated. This is primarily achieved through object oriented programming $\left(\mathrm{OOP}^{16}\right)$ within the particular API.

Commonly identified as associative parametric design ${ }^{17}$ packages, these more complex tools are intended to take account of both the act of design and the ability to utilise programming methods. The interface to these algorithmic representations of geometry is indispensable to creating algorithms which can explore 'undrawable" ${ }^{18}$ forms and handle complexity in a way not possible with direct manipulation. There is also a gradient of complexity between the general and explicitly parametric programs. The Autodesk 3DSMax user may take advantage of scripting, and complex parametricised systems, but their freedom is limited by their

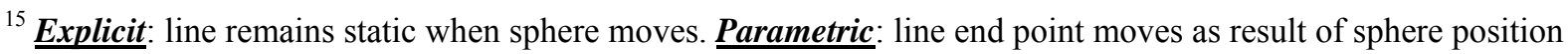
change, and sphere location changes as result of line length increase. Associative: sphere radius, line position, cone size, and cone position change as a result of box length increase and vertex position expressed in a 'deeper' algorithm.

${ }^{16}$ Schach (2008)

${ }^{17}$ The term associative design, which builds on the understanding of associative geometry, is generally interchangeable with parametric design (Aish 2003).

${ }^{18}$ Aish (2009)
} 
understanding of the scripting language, while the user of the explicitly parametric Bentley GenerativeComponents ${ }^{19}$ has a deeper set of tools (and the ability to use custom tools) in which to express algorithmic relationships between abstract geometry and explicitly architectural objects. 3DSMax uses algorithms to define standard objects, which is faster than creating objects from scratch, but it does not provide a 'deep' interface permitting direct access to the underlying algorithms which control the objects. McCullough (1994) describes the distinction in interfaces and control of algorithms, in part, as density of notation.

The term Digital Parametric Design Tools (DPDT's) thus encapsulates both the general digital tools used in design such as MSExcel, advanced three dimensional associative design programming tools such as Generative Components(GC) (Aish2006), Grasshopper (GH), Digital Project (DP), and everything in-between such as 3DSMaxDesign and even Google SketchUp. Consequently, the term parametric design can be understood as relating to architecture in three ways: as a description of implicit relationships of constraints within general problem solving; as a general term applied to executable programs which adapt to differing inputs and options; and as explicitly parametric design programs such as Grasshopper or GenerativeComponents which employ programming methods. These can be summarised as design generalisations, digital generalisations, and explicitly parametric applications of the term 'parametric' in architectural design. The way DPDT's are typically used in architectural design is illustrated in fig 5.

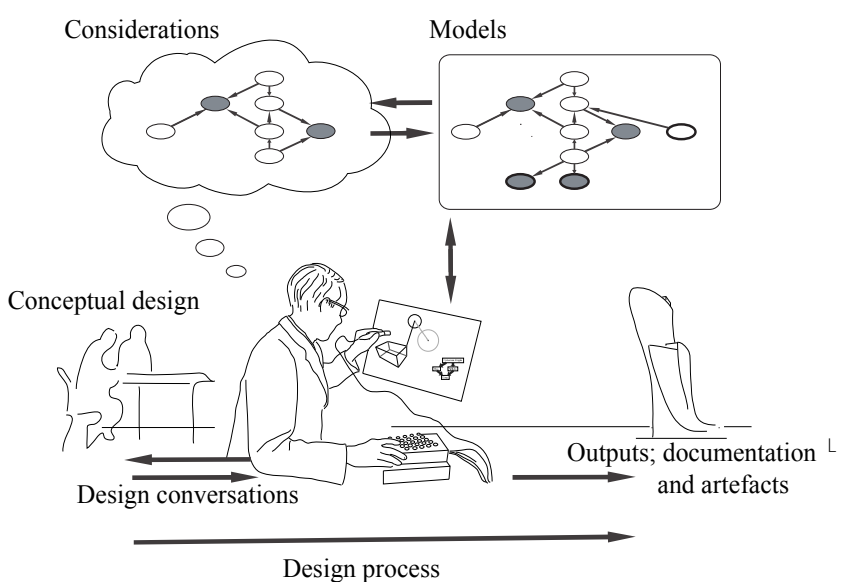

Figure 5: Cyclical architectural processes (in part after Sutherland):

The design processes is represented as a series of tasks from conceptual design to built artefact (left to right); The modeller translates and encodes design moves from iterative, reflective conversations into a notation which is useful to the medium and design outputs.

\footnotetext{
${ }^{19}$ Generative Components (Aish 2006)
} 


\subsubsection{The problem with parametrics}

Jabi (2007) summarises his response to Johnson's ACADIA group discussion topic regarding digital design "What are the grand challenges in our field?" with the following:

"... I think the greatest challenge facing us is that our field is plagued by fragmentation with no clear research taxonomy, no rigorous methods of peer-review and evaluation, fragmented and inadequate software, and poor physical settings. If you are looking for one great challenge, I would posit that our design processes are being poorly supported by brittle and antiquated software interfaces and mechanisms."

Jabi's comments suggest that obstacles outlined within theoretical literature are not well understood or researched. The reasons for could be varied, but one issue seems to be the tendency of the literature to focus on the positive outputs and omit many of the limitations and inherent difficulties. This suggests that the practical implementation of DPDT's could be better understood through deeper and more frequent inquiry. Further, Jabi (ibid) suggests that there is also an issue of the "oversubscription of parametrics" in architecture, in which there is a risk that the upcoming generation of designers may use tools intuitively and in a ubiquitous manner but apply a skin deep understanding of the base principles of the actions they instruct a computer to perform. This can lead to a situation where form follows fashion rather than function; there is a risk of institutions and practitioners becoming seduced by visually oriented work and parametric design which focuses on modelling randomly generated form rather than designing form based on well-understood parameters.

In contrast to theoretical issues focused on assessment of the forms produced by the application of DPDT's, the difficulties focused on in this thesis on the operational level of application are caused and affected by a mix of process requirements, the craft level of modellers, and the particulars of the medium. The general problems with the implementation of parametrics can be classified into two broadly defined areas: those related to the extra analytical work that parametric modelling entails, and those related to the necessity to remake or totally rebuild the algorithmic structure of a model from time to time. The general problems with the integration of parametrics is more varied and more difficult to classify. 


\section{Extra work}

Generally, the use of DPDT's over so-called 'one-off' direct modelling methods involves an increased workload in which negotiating multiple, interdependent, and sometimes circular constraints can be challenging ${ }^{20}$. One of the most evident aspects of translating design ideas and constraints into parametric models is the act of translating an abstracted representation (or idea) into an explicit, precise model in an accommodating manner. A notorious example of this is Gehry's translation of sketches and physical models to digital form, (Shelden 2002) where the parametric model acts as an intermediary; translating design intent through development and construction. Christenson contends that media can resist such translations ${ }^{21}$, and Aish comments on the act of associating the subjective evaluation and the formal descriptive system and vice-versa as inconsistent ${ }^{22}$, as the thinking is perhaps counterintuitive to the widespread methods in design.

The first genuine obstacle in parametric design is that designers do not tend to think or work parametrically, and that parametric thinking is an inherently more involved process ${ }^{23} 24$. The modeller often is required to translate a diverse set of design decisions and constraints into the model, and design decisions which could be effectively described with a 'napkin' sketch can take a great effort to encode / accommodate within a parametric model. This problem is often made worse by the programming methods used within advanced tools, as there is an additional cognitive load ${ }^{25}$ for the designer or modeller in understanding how to represent the relationships within the tool as well as defining the relationships themselves.

\footnotetext{
20 "The interplay of many constraints can lead to circular dependencies that make design exploration a challenge as any change causes ripples throughout the entire design construct." Killian (2006a,b)

21 "specific media resist certain kinds of translation" Christenson (2011)

22 "'This is the shape I have sketched, but how to I formally describe it' versus 'This is the formal shape description system I am using, how do I harness and control this system to create the shape I like"” Aish (2005). 23 '" Parametric thinking' and 'algorithmic thinking' place the processes which one typically associates with computers and software in one's mind. ... If I'm thinking algorithmically, I'm not necessarily thinking of a form which will meet my design criteria, but instead thinking of the rules which will meet my design criteria... " Katz (2010)

24 "Parametric thinking is a way of relating tangible and intangible systems into a design proposal removed from digital tool specificity and establishes relationships between properties within a system. It asks architects to start with the design parameters and not preconceived or predetermined design solutions." Karle and Kelly (2011)

${ }^{25}$ Additional cognitive load Maleki and Woodbury (2010)
} 


\section{Multiple models}

A suitable model incorporating the appropriate parametric relationships may take several iterations to develop as the consequences for a particular configuration often only become apparent after it has been constructed. This leads to a need for multiple model iterations to respond to the rethinking of the design problem and its constraints to allow for changes in the design solution, often carried out through a trial and error process of assessing comparative methods. Designers are used to refining their designs through constant review and revision including 'going back to square one' with a form or plan, but parametric design adds the extra cognitive load of 'going back to square one' with the defining equations or parametric relationships.

Burry (1996) provides an example of the requirements of a model changing so the the structure needs to be reconfigured or remade from base principles. Burry (ibid) recounts how parametric models for Sagrada Familia elements were rebuilt upon finding of specific constraints which nullified the usefulness of an existing model. Hernandez (2006) further describes some of the thought processes involved in developing iterations of parametric models of the famous Gaudi design. One can observe from this that a relationship that enjoys a wide range of variability that can be promising at the time that it is implemented can become unexpectedly constrained by later and apparently unrelated decisions. The designer can also find themselves in a dead end, especially if they have not prepared a clear structure with which they can assemble the parametric model (Burry and Murray 1997). So the designer needs to organise the structure of a model so that it can be represented within a parametric medium. However, the process of design can change the perception of the design thus clarifying this structure and bringing about a rethink and improvement of the previous model structure.

If a model cannot accommodate a change by a user, such as the size, rotation, or geometric transformation, then it usually needs to be restructured. A simplified example of this from Burry's (2003) illustration can be seen in fig 6. Burry (2008) implies that this is both an inherent part of the parametric design process, and a limiting factor ${ }^{26}$; thus arguing that it is easier to develop parametric models towards the end of a design process rather than at the

\footnotetext{
26 "can we sensibly model architecture using parametric modeling if inevitably the process involves erasure and redrafting[?]” Burry, J. (2008)
} 
conceptual stage $\mathrm{e}^{27}$. Kolarevic has a slightly different view and states that multiple models are required because "The designer simultaneously interprets and manipulates a parametric computational construct in a complex design process that is continuously reconstituting itself". However, this is both a problem to be dealt with through dexterity and proficiency and a source of unpredictable and unexpected poetic and creative discovery (Kolarevic 2008).
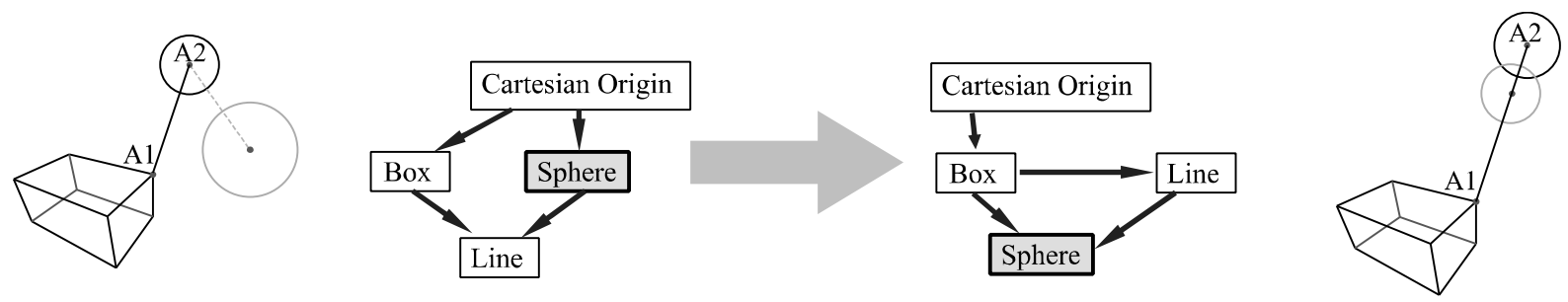

Figure 6. Changes to dependencies in a parametric model; a model needs reconfiguring in this situation because a modeller realises that the model is more meaningful if the sphere position is controlled by a distance and a vector rather than by simply placing the sphere in a different location.

\subsubsection{Heuristics and DPDT's}

Woodbury, Aish, and Killian (2007) suggest that patterns can hold an answer to the problem of degrees of freedom and the arrangement of meaningful variation; "A pattern typically comprises a name, a problem description, an abstract solution, and a discussion of consequences" ${ }^{28}$ in a manner which traces back to Alexander's Pattern Language. This helps the parametric modeller because "patterns act as informal objects-they provide a structure whereby edits can be more readily understood than by examining an undifferentiated complex model" (ibid), further suggesting that novel, or loosely defined problems produce extra difficulties in modelling.

Design usually involves modelling of some type where the goal is to produce a physically constructible artefact through a formal shape description system. The difficulties outlined above relate to the problem of understanding a design situation well enough that it can be represented algorithmically. Users of digital modelling tools can make use of heuristics

\footnotetext{
${ }^{27}$ Relational models “... a good workflow for modelling a project once past the very volatile conceptual stage but where the design is still undergoing significant refinement and iteration" (ibid)

${ }^{28}$ Woodbury, Aish, and Kilian (2007a)
} 
within algorithms to achieve this. Heuristic methods ${ }^{29}$ are tools, or rules, which allow problems to be solved easily or in a short time frame. A simple explanation of heuristics is that they are a variety of intuitive methods which allow a solution to be found using a rule of thumb, a methodology, or some other device through somewhat simplistic means that do not exactly relate to the full detail and contextual setting. One broad, and perhaps unsatisfactory, definition which follows this line of reasoning is that “...heuristics are strategies using readily accessible, though loosely applicable, information to control problem solving in human beings and machines" (Pearl 1983) ${ }^{30}$. Rowe (1987) in his analysis of design procedures assesses heuristics as a term which "is applied to general kinds of procedures for guiding the search for solutions"(ibid p.75), while also suggesting that no general theory exists on the subject. He also suggests that there are numerous definitions ranging from explicit 'decision rules' in an algorithmic sense to heuristics being used by designers as being "quite subjective, having evolved from prior personal experience" ${ }^{31}$, suggesting that experience-based techniques or even metaphors or can be seen as heuristic devices. Further possible interpretations of the term exist, and although several definitions may be applicable on several levels of design conceptualisation and problem solving, this thesis regards heuristics in relation to the computer science adaptation of the term as "denoting a rule of thumb for solving a problem without the exhaustive application of an algorithm. „29

Heuristics can be utilised without a deep understanding of their origins. One problem with parametrics revolves around this point: that on one hand, heuristic solutions allow for a more efficient application of parametric tools, but on the other it can oversimplify the system under consideration such that the understanding of base principles can be more difficult. Turkle (2009) highlights an example of a comparable phenomenon during the development and emergence of human-computer interfaces at MIT in the 1980's. The advent of Graphical User Interfaces (GUI's) meant that students were no longer required to understand how to perform programming from base principles in order to use software in their learning and research activities. No longer did students need to understand how a computer worked; just that computers do work, and presuppose that the results are realistic and credible. A further

\footnotetext{
${ }^{29}$ Collins Dictionary (2011): (from Greek heuriskein "to discover”) “ heuristic [hjoə'ristrk] adj

1. helping to learn; guiding in discovery or investigation

2. (Social Science / Education) (of a method of teaching) allowing pupils to learn things for themselves

3. (Mathematics) (a). Maths Science Philosophy using or obtained by exploration of possibilities rather than by following set rules (b). Computing denoting a rule of thumb for solving a problem without the exhaustive application of an algorithm a heuristic solution"

${ }^{30}$ Pearl (1983) p.vii

${ }^{31}$ Ibid, p 76.
} 
example is the advent of the 'mouse' peripheral device; command prompt typed commands were not a necessary method of utilising the technology. With the mouse and the GUI, the user can think they are working at a fundamental level, because they understand the interface, but they are removed from access to the actual activity; a heuristic device may be accepted as a true model of reality, rather than an approximation. Most geometric design programs have highly developed mechanisms behind the scenes, but the newer intuitive interfaces restrict access to the majority of users. An example of this can be seen in the way that prevalent platforms within the $\mathrm{BIM}^{32}$ grouping of software tend to model rather than design, and push a designer to simplify and standardise a design ${ }^{33}$. At times parametric design can mirror this type of behaviour; for example when a modeller finds a subroutine for calculating an aspect of the problem at hand from an online source which contains complex algorithms beyond the capability of the modeller: it is easy to use in a standard manner through copy-paste actions but it can be difficult to edit specifics at the base principle level to adapt to the particular problem.

Thus, heuristic devices can be seen as both a key aspect of DPDT's which makes them efficient and useful to designers, and also a driver of naive methods and understanding which can impact the problem solving processes in both positive and negative ways. With the advent of mass-uptake of DPDT's one could say that, generally, the tools are easy to apply, but difficult to understand and manipulate at a base principle level. Understanding the pitfalls of a set of processes allows for deeper understanding of effective methods for design integration allows tools to be used strategically, and conversely, the lack of understanding has a negative impact on this integration. It is important to better understand the role of parametric design tools as the mass uptake of these tools gather pace. A greater understanding of parametric problem solving methods is needed to gauge which kind of problems can be solved with the development and refinement of models, which should not, and which cannot.

\footnotetext{
${ }^{32}$ Building Information Modelling (BIM) include AutodeskRevit, GraphisoftArchicad and the like.

${ }^{33}$ AutodeskRevit, for example, can accommodate imported ACIS solid geometries (for some reason only ACIS v3.0 or earlier, not the current v9.0) but does not include the tools to create an exhaustive range of complex solids.
} 


\subsubsection{Why Use Parametric Design?}

The usefulness of parametric tools in the area of capturing design relationships ${ }^{34}$, design documentation $^{35}$, performative simulation of building facades ${ }^{36}$, and geometric complexity ${ }^{37}$ has been proven and illustrated in contemporary practice. Digital generalisations of parametric tools have been widely hailed ${ }^{38}$, but also noted is that they increase the complexity of the design process ${ }^{39}$ and can inhibit design freedom ${ }^{40}$. Considering these examples, parametric design in this research is conceived and carried out within broader design processes. The case studies mentioned above place parametric design within contexts that explore it ability to enable exploration while recognising that it has the potential to restrict the options for integration with the design problem.
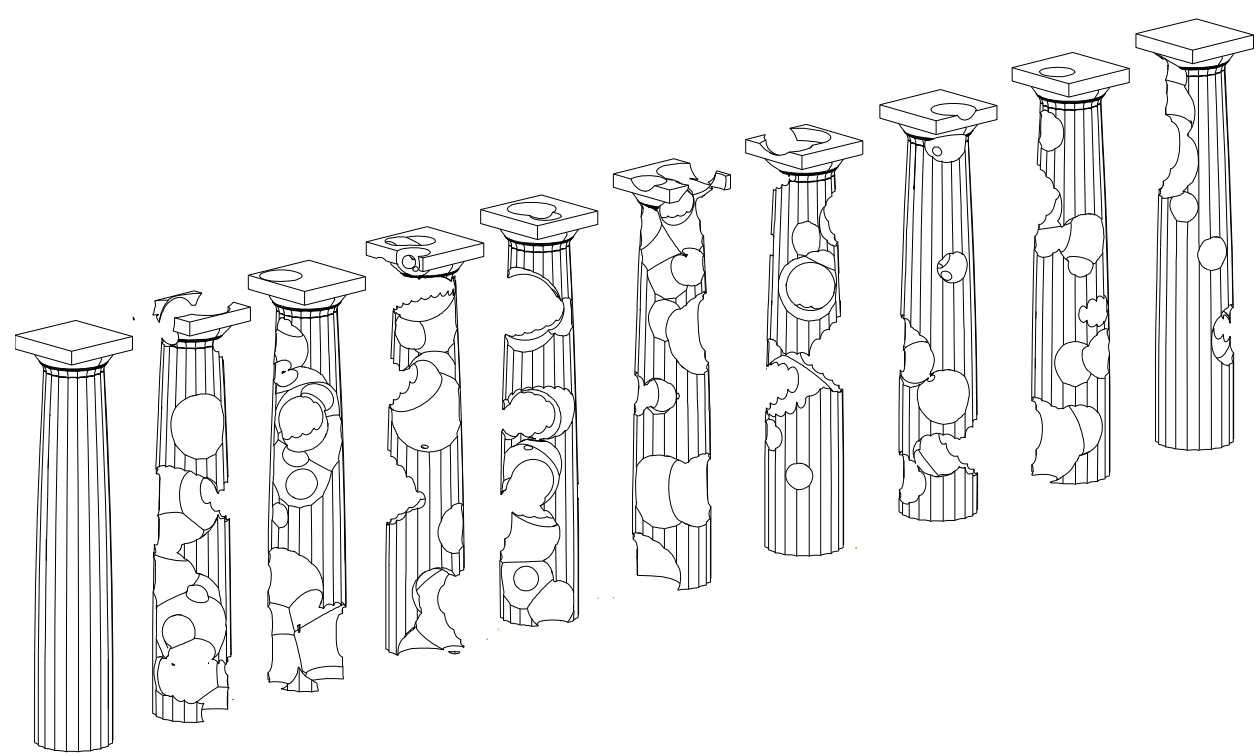

Fig. 7. Illustration of possible (and relatively superfluous) variation;

complex constructive solid geometry variations which are computationally expensive; complexity which would previously have not been possible. $\left(4.2 \times 10^{14}\right.$ possible variations within this schema.)

The core benefit of parametric design is in the (sometimes very large, incalculably high, or near infinite) set of solutions which may be generated and assessed. This can be understood as the solution set or solution space. One of the most apparent issues with this is the

\footnotetext{
${ }^{34}$ Woodbury and Marques (2007b), Aish 2005

${ }^{35}$ Shelden 2002, also Glymph et al. (2002)

${ }^{36}$ Greater London Authority Headquarters (2002) also the Swiss RE building (2004) Foster \& Partners et al. also Hesselgren et al 2007 Bishopsgate tower

${ }^{37}$ Shea et al. 2005 ,

${ }^{38}$ Schumacher 2008, Burry 2011, Woodbury 2010

${ }^{39}$ Aish2005, 2009

${ }^{40}$ Mueller and Johnson 2000
} 
relevance or desirability of possible variations. The question of meaningful relationships highlighted through variation can be problematic. On one hand, numerous variations in a design may be pointless from the outset, but on the other, meaningful variation may not be evident upon initiation, and exploration of the unusual or accidental can help develop design solutions. An example of parametric variation (possibly meaningless variation) of deconstructed column solids is illustrated in fig 7. Essentially, an increased solution set allows for exploration through higher levels of expression and control: Aish (2009) states the following regarding parametric design:

"The designer need no longer focus on designing a specific artifact (or configuration), but rather, he has the opportunity to externalize the underlying "design logic" and use this to explore a whole range of alternative solutions. Design Computation also suggests a different approach to the development of software applications. Instead of presenting a completely comprehensive "view" of the design domain, the design application effectively becomes a platform that presents a more general abstraction of geometry and composition. The designer (with computational skills) uses this platform to create the final, customized layer of application logic, specific to the design problem at hand."

Parametric design and the use of parametric models requires both an understanding of the tools themselves and also the development of the technical skills which these methods require. With explicitly parametric tools these requirements can be seen as revolving around the application of programming methods ${ }^{41}$ and the design of programs which solve particular problems. Because programming methods are not typically taught to designers, this means it generally takes them more time and effort to build parametric models than creating singular configurations. The variability of the scope or 'depth' of the parametric model means that there can be seen to be a gradient between 'parametric' and 'mechanical' models. The more variables and situations a parametric model takes into account (the 'depth' of the model) the longer it takes to create and refine. This is also known as degrees of freedom (Anders 2003, Burry 2006) or degrees of flexibility. Burry's description of parametric modelling as design of design (Burry2003) highlights the issue which arises during such a process; that the design of a parametric model is a design problem in itself. This is further reinforced by Fischer's observations regarding the creation of design aids such as parametric models as a process of “designing tools for designing tools for designing tools ..." (Fischer 2008 ) which have their

\footnotetext{
${ }^{41}$ Swinson 1982, Maleki and Woodbury 2010
} 
own requirements and techniques which are somewhat separated from the original design problem.

The process of applying explicitly parametric design tools to architectural problems relies on programs which allow defined functions and parameters to control geometry rather than a more explicit representation of objects. As such, following the formation of initial associations, the design solution can be progressed by changing the values and relationships of parameters at different stages of the design process to both refine the topology of a solution or to reorganize the relationship between parameters and geometry. These functions and parameters define the creation of the required geometry through algorithms within the contextual language, facilitated by analysis of the design problem by the modeller. The functions can be understood as the associations between parameter values and the parameters are organised to be dependent or independent. The independent variables are in essence the core of the user interface of the model as they tend to be under-constrained and allow flexibility by updating dependent variables (and geometry) through logic chains. It has been noted by Burry (2006) that when creating parametric models 'from scratch' the specifying of values for parameters is relatively straightforward, but the tasks of refining the associations or functions is both a necessity of process and a time consuming and difficult task. These refining tasks are the job of the modeller as illustrated by the PhD's from Shelden (2002), Killian (2006b), and Hudson (2011).

The answer to the question "Why use parametric design?" is quite simple: to allow for variation in the set of meaningful, viable, and calculable solutions through deeper control and expression of geometry. Aish (2005) states the following regarding the geometric abstractions of computational design tools:

"What becomes apparent is that we are not designing the geometry of the artifact, but rather we are constructing a 'control rig', some geometry that will never be built or seen, but which indirectly controls what will be constructed and experienced. It is the development of this sense of 'indirection' or 'control through geometric dependency' which is emerging as a key design skill. By building and exercising these systems of geometric dependency we are able to explore variation in design, indeed to explore the solution space, and to discover and validate the configuration that will finally be constructed." 


\subsubsection{Design as Constraints}

Design in this thesis is viewed as an act (verb) of designing which is viewed as a problem solving process in which the description and requirements can be challenging to pin down. The processes involved and the solutions which emerge from design problem solving have particular characteristics fairly unique to architecture. For traditional architectural problems the most apparent attribute is the uniqueness of the setting: architecture is situated in a particular site which generally (but not always) is given as a priori knowledge at the early problem description stage meaning configurations of physically constructible artefacts tend to be one-offs and unique. Another quality (and quantity) of the design context which is easy to comprehend is the stiff constraints of a building site boundary. What makes architectural design problems unique is the mix of straightforward and abstract variables and constraints; for example a design solution may simply be too 'ugly' to meet the aesthetic constraints or some other poorly defined criteria. Typical design problems can be categorised as wicked problems (Rittel and Webber 1973) because there are no 'best' or optimum solutions, because many solutions meet the criteria and constraints of the design problem. The number of possible solutions can be innumerably high, as the number of independent variables in the design solution of even relatively straightforward configurations can be high.

One simple illustration of this complexity is a simple rectilinear 'box' placed in Cartesian space with degrees of freedom allowing size, location, and rotation. Already, to describe this simple object there are nine 'dimensions' of freedom ${ }^{42}$; that is an $\mathrm{x}, \mathrm{y}, \mathrm{z}$ variable for each of the following: location, orientation and size of the box. In real space where quantities can be subdivided infinitely, we end up with a more than infinite number of possible positions; in fact there are nine 'infinites' to this situation (or nine infinitely sub-dividable dimensions). The solving of this type of problem is performed as a search of the possible solutions (instances), where heuristics allow workable limits to be placed on the number of considered solutions. For the box, this could be a constraint such as maximum or minimum box volume or a subdivision of length and angle to 'packets' of measure (such as 1 degree or $100 \mathrm{~mm}$ ). This makes the finding of solutions workable because the solution space is restricted. In describing a simple box with a fixed length, breadth, and height of between 0.1 and $1 \mathrm{~m}$ and within a $10 \mathrm{~m}$ cube with length and angle subdivisions of $0.1 \mathrm{~m}$ and 1 degree there are around $4.6 \times 10^{14}$ viable configuration instances (fig 8.). If the box is of fixed size and constrained to

\footnotetext{
${ }^{42}$ N dimensional Mahdavi (2002). Dimensions Penrose (2005),
} 
a single axis of rotation then the number of possible configuration instances is $3.6 \times 10^{7}$ ( or fewer configuration instances by a factor of around 129 million).
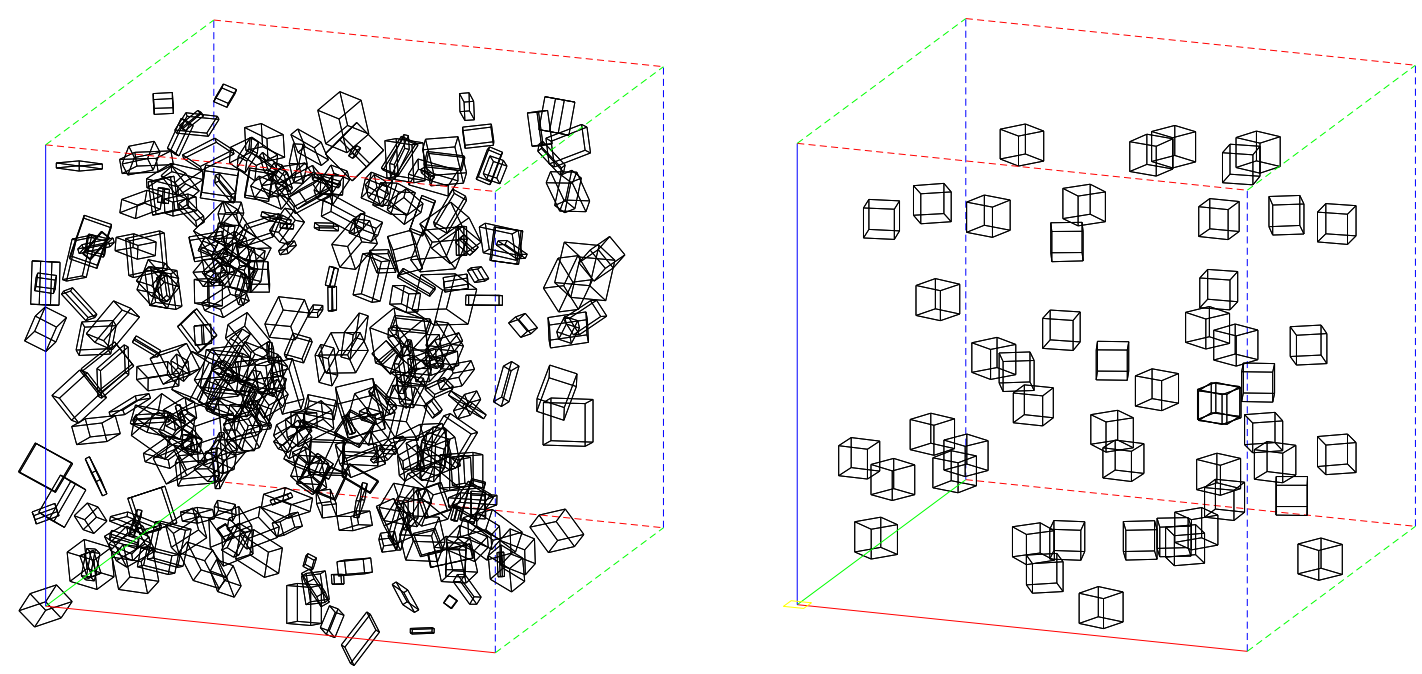

Figure 8. Possibilities of unconstrained and constrained box generation;

(a) 9 degrees of freedom; $4.6 \times 10^{14}$ instances (b) 4 degrees of freedom; $3.6 \times 10^{7}$ instances

Within architectural problems the number of variables tends to be higher than 9, but variables can be organised to be dependent on (or constrained by) 'top level' explicitly defined variables. This constraining of variables from the top of the dependency chain assists problem solving by confining the solution space to workable and logical limits. An obvious example in architectural production is a budget; which will immediately rule out certain solutions which are simply too expensive. A different example of this would be the design of Greek Doric temples which were in essence set out through determining the length as the 'top' of the dependency tree and following a set of principles within a pattern which defined elements of the structure from this ${ }^{43}$. Both examples use a form of heuristics by using existing and calculated information to inform design processes to make the search for solutions workable.

The implication of having a huge number of instances to choose from is that the way problems and solutions are developed requires a flexible, iterative, reflective process. The design process seeks to moderate multiple assessment criteria as the design progresses. Schon's (1983) constructivist analysis of design as reflective practice where design activities are continuously framed and re-framed is a relevant framework for understanding this. The search of the design solution space is fundamentally iterative and can occur through diverse means. The problem and solutions are developed in tandem through reflection in action

\footnotetext{
${ }^{43}$ See Virtuvius Pollio, De architectura, Book 4 Chapters 3 and 4 (Temples) endeavour to explain this logic.
} 
(ibid). Both Asimow (1962) and Lawson (1994) view design as an iterative three stage process of analysis, synthesis and evaluation. Gero (1990) refers to Asimow's analysis and constructs a modified model which accounts for these stages of design and the behaviours in which design elements are assessed and iteratively rethought on different levels. This socalled function - behaviour - structure model represents design knowledge development and allows for reformulation of the initial functional requirements through diverse means; such as reformulations of the expected function of the design based upon the performance of a proposed structural outcome. Hence this model can be beneficial to consider as it matches what can be observed in the diverse set of processes of architectural design; including the possible "it is too ugly" argument for iteratively reworking design moves and assumptions. There are several abstracted models of design process theorised within the field and these occur at different levels. Generalising, there is common acceptance of design as a sequence of situated reflective acts which is required to negotiate multiple objectives within a large set of possible solutions.

\subsection{Hypothesis}

The literature outlined above helped define the general research question:

What are the characteristics of some of the observed issues and obstacles revealed in the practical application of digital parametric design tools?

What can be observed from practice and alluded to from literature is that explicit parametric modelling (associative geometric parametric modelling) is generally technical, difficult, and presents problems to the modeller which are removed from the wider design problem. Also, the development of a design problem/ solution pair is encapsulated within an iterative process of 'reflection in action'. This means that the formal logic which underpins the design solution is an evolving 'plan' or schema. When a sub part of this problem is modelled explicitly then the formal logic of the schema must overcome several difficulties to become useful and integrated within the design process. Therefore, the hypothesis of this thesis is as follows:

1. That there are observable obstacles inherent in the use of digital parametric design tools which impact their use as architectural design aids. 
2. The obstacles can be grouped into two generalised divisions; the obstacles of tool interface required to arrange models, and the obstacles faced when arranging a model to provide useful outputs to an iterative design process.

3. Education/training plays a role in conjunction with these two obstacles.

4. The core obstacle to integrating DPDT's is the ability of the modeller to adapt one schema as the design is explored and requirements change, and thus, the effects of tool interface and design process interface can be accounted for and anticipated to allow smoother and better integration within the design process.

5. The effects of the core obstacle can be minimised by following strong patterns which limit the unknown quantities. Less well defined problems and model patterns encounter greater obstructions.

\subsection{Method}

In this section several relevant methods are critically assessed against the research objectives. Literature search, case studies, and Linkographic methods are assessed for their suitability to explore the research question and address the hypothesis statement outlined above.

The field of computational design as defined by Mueller (2009a, 2011) is a field which is currently rearranging itself in response to its omissions and new potential roles in regard to useful outputs within architectural design processes. Of the three axes identified by Mueller (ibid) 'practice models / supporting technology' is the aspect of computational design which is undergoing growth due to increasing interest within institutions and practice, and is the sub-field which his thesis is concerned with ${ }^{44}$. The crossover of methods with the field of design cognition and general programming has enabled a greater depth and breadth of possible research to occur, whilst allowing the avoidance of well defined overarching theoretical models. What has been increasingly occurring is the growth of what one might call 'practical literature' as opposed to 'theoretical literature', perhaps because of the lack of clear taxonomy (Jabi 2007), and growth of a defining body of research ${ }^{45}$ which tends away from wholly theoretical concerns. Shelden (2002), Killian (2006b), and Hudson (2011) mainly focus on case study and design experiments alongside and relating to literature. Hudson maintains that the distinction between theoretical and practical literature in the field

\footnotetext{
${ }^{44}$ The other axes are design investigations, project lifecycle, and practice models/supporting technologies as stated in section 1.0

${ }^{45}$ Bhatt et al. 2006, 2008, 2009
} 
is valuable in framing methods such as participant observer case studies, whilst understanding the limitations and tendencies of such research outputs. Thus, one can view research in the 'practice models / supporting technology' axis of the computational design field as requiring both a combination of research methods and a practical rather than wholly theoretical distinction to be made of literature.

If theoretical literature in the field can be defined by publications such as Schon (1983), Mitchell (1990), and Frazer (1995) as stimulating movement towards clarifying design philosophies, then practical literature can be seen as the 'groundwork' which influences the theories within design. In the sense that there is a weak taxonomy within the field, these theoretical texts are useful in creating attraction to and concern for computational design, but less helpful in influencing practical applications. An issue with theoretical texts is that the models and theories outlined can become somewhat old fashioned (or at least become less important) as technology and practice shift ${ }^{46}$, whilst practical literature emphasises the specific design problem at the 'coal face' and thus reflects a snapshot of the diverse and highly specific problem solving methods used at the time. Whilst the relevance of design theories may expire, documented 'snapshots' of practical problem solving activities retain some usefulness over time; the actual methods for solving problems may become obsolete or unfashionable, but the design approach and considerations can continuously be referred to in a relevant manner. An example of this is Alexander's breakdown of a specific design problem of a village layout in Notes on the Synthesis of Form (1984), which still holds meaning even if Alexander's theories have lost popularity. Following an upsurge in publication of practical literature in the last decade, the models and theories of design and design computation can be examined against practical methods and be found to be less overarching and more specific to particular approaches. This may in part be because the traditional boundaries and methods in design are being blurred and reconfigured; not in the least by developments in technology.

Practical literature tends to originate from industrial or business activities, but also intersects with the outputs of academic institutions; and in this thesis mainly comprises reference to

\footnotetext{
${ }^{46}$ One example is Fischer's reassessment of Frazer's Evolutionary Architecture as reflecting previous, possibly superseded view which can be shifted once one considers more recent developments in the field of cybernetics, amongst other things. Fischer (2008)
} 
conference papers and postgraduate research. Much of the practical literature in the field can be found in the CumInCAD database which at its core consists of conference proceedings of ACADIA, eCAADe, CAADRIA, ASCAAD, SiGraDi, CAADFutures, and DDSS ${ }^{47}$. Practical literature can be seen as having an advantage over the theoretical in that it disseminates accounts of processes which would otherwise be challenging to document and categorise within theory. It is common that multiple projects are recounted, which allows limited and specific analyses to provide a general account of process phenomena. There is an issue with the limited ability to reproduce findings of case studies in design as compared to other fields. This can be because the logics underpinning design problems (and design problems which are wicked problems) invariably change configurations in different contexts, and this logic can be modified by abstract knowledge including opinions, experience, and aesthetics.

Another issue with practical literature is that the full set of processes undertaken are not described in detail, or are completely omitted in the context and format of a conference paper. The scope of such formats means that practical literature tends towards summary of processes rather than in-depth analysis. This restriction is generally not the case in $\mathrm{PhD}$ theses ${ }^{48}$ in the field as there is a greater focus on appropriate alignment of detail all the way to theory combined with a broad approach to the investigation. Even without this restriction, PhD's in the field omit a certain amount of detail such as dead ends, specific problems with software and more anecdotal points which are not directly relevant to the narrative of the thesis analyses, but which could be helpful to the author's fellow parametric design practitioners. Research traditions in architecture and the sciences have historically shied away from inclusion of such participant-observer evidence, although Woodbury et al. (2007c) suggest that the parametric design subfield could benefit from such an approach. In some cases practical literature can tend towards a form of promotional advertising, whether academic, industry based, or part of business activities. Such literature needs to be sceptically assessed when considering parts of the process which have been omitted. A further issue is the overstating of findings; this can occur when the generalisations of practice based writing seeks to produce frameworks for theoretical concerns. Practical literature may draw from theoretical, but can tend to a bias view of the relevant issues, and by definition does not extensively question theoretical assumptions. The goal of providing a theoretical framework may stretch the generalised findings of this type of literature to encompass aspects of process

\footnotetext{
${ }^{47}$ See bibliography for more information

${ }^{48}$ Such as those mentioned above (Shelden, Killian, and Hudson)
} 
which are largely specific. An example of this would be the extrapolation of theoretical frameworks from qualitative accounts of processes ${ }^{49}$ (note). For this reason, practical literature has a greater risk of falling into the category of position papers which essentially explain an opinion through selective use of sources and omission of results which overcomplicate or disagree with a certain position. Despite this, the chief benefit of practical literature is that it provides an insight to how the processes concerned are actually used in real world examples rather than speculative insights which can tend to be seen as 'out of touch' with reality.

Case studies provide a grounded method to examine issues in the field which differs from a literature review or analysis of theoretical implications of software potentialities. A key advantage of the approach is that over-generalised understandings can be avoided if the analysis is based solely on specific consequences and approaches within the case. Conversely, the findings are less helpful when assessing wider design processes beyond the case in question. Case study methods in the design field have greater utility when multiple, complementary cases are accounted for and contrasted. Events of interest can be few and far between in case study research and generalising from them requires in-depth knowledge and familiarity with the cases in question. This can mean that accurate accounts require the researcher to be a participant-observer, matching Woodbury's (2007c) argument.

Undertaking multiple cases raises problems of research scale. A three year minimum length $\mathrm{PhD}$ thesis may provide sufficient time for this type of effort, but it is difficult to reduce to shorter format research outputs. Some well known examples of PhD's which contain detailed case studies are Killian (2006) and Hudson (2011), where multiple cases are distilled and contrasted to illustrate the theoretical implications of problems faced by practice. Singular case studies within conference papers are also within normal practice. In architectural research these can take the form of analytical cases to reveal existing systems and relationships ${ }^{50}$, as theoretical devices ${ }^{51}$, or the testing of a practice oriented design aids ${ }^{52}$.

In Goldschmidt's (1990) Linkographic method designers' interactions are observed, categorised and explicitly represented as points and lines (fig 9.). The graphical

\footnotetext{
${ }^{49}$ An example of this might be Chang and Chang (2006) one-dimensional investigation of physical folding following reference to the Deleuze (1988) concept of fold.

${ }^{50}$ Some examples Talašová (2009), Anay (2010)

${ }^{51}$ Fischer et al $(2003,2011)$, Feng (2010)

${ }^{52}$ Schoch (2010), Wong and Cheung (2010)
} 
representation provides a map of the design moves (nodes) and the links between them (connections), which are drawn on a triangular grid. The overall process direction of the diagram flows from left to right and allows the impact of the design moves to become most apparent as the path of links connecting nodes appear as triangles in the vertical dimension. The relation between design moves can be seen clearly, and be used to assess productivity, density of moves, and the impact of early design moves overall. The design procedures which are often abstract in nature can be recorded; these include stated opinions of designers, and casual conversations, design sketches and much of the abstract information and tacit knowledge shaped during the design process. Linkography can be used to examine issues within design processes such as entropy and richness (Kan and Gero 2005). Gero also applies the Function-Behaviour-Structure (FBS) design analysis model to Linkography to capture a larger set of critical design moves (ibid).
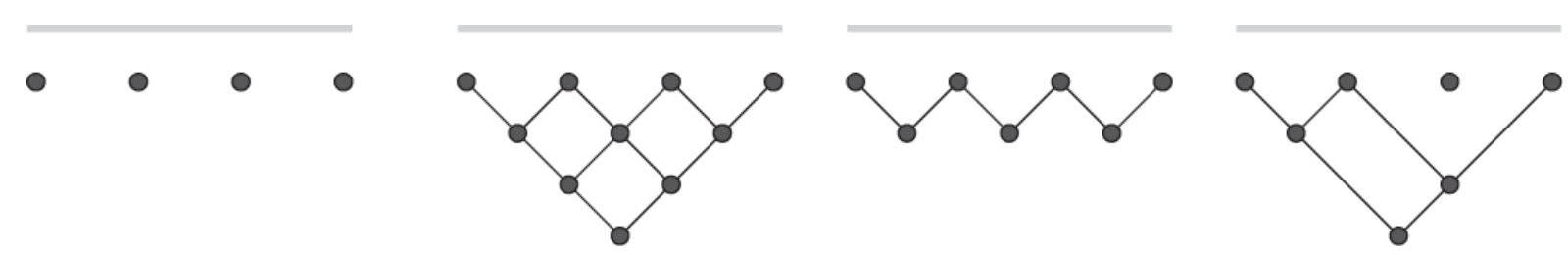

Figure 9. Possible design moves represented in Linkographs after Kan and Gero (2005) (a,b,c,d):

(a); moves not related, ideas not converging, process not rich.

(b) all moves connected, process is integrated, ideas may be fixed.

(c) moves related only to previous steps, process progressing but not developing.

(d) move 3 not related, central idea present as well as some diversification, process is rich.

The main benefit of Linkographic analysis is that the fine grained details of design moves can be documented in a graphical manner. The graphs resemble node graphs of logic which are similar to explicitly parametric design programs, but focus on design moves rather than objects (encompassing geometric and non geometric elements). A drawback of this approach is that it requires that the design process being graphed must lend itself to a group environment and requires an effort to record conversations, sketches, and a multitude of interactions some of which are hard to summarise. The complexity of the design problem also affects the usefulness of this method of analysis, and the effort required to document these processes can be many multiples of the effort expended solely on the design task. Another drawback is the lack of familiarity with this method and limited uptake from the research community. Linkographic methods are well suited to an analysis of the difficulties of using DPDT's, but would better suit a more in-depth study and are outside the scope of this research. 


\subsubsection{Selected Approaches}

Theoretical literature is useful in outlining some aspects of the research field but is limited by several factors. The lack of clear taxonomy is one of the key problems with literature in the computational design field which can make a detailed literature study ineffective in pursuing analysis of overarching design models. There is information and knowledge which theoretical literature cannot account for, or is generally omitted within the body of research. The relative difficulty of using parametrics is one such area of knowledge. For this reason, models of design from selected theoretical literature in this thesis are not taken as a priori knowledge on which to base case studies. The characteristics of practical literature allow for a bottom up investigation of difficulties within the use of DPDT's; and these can be compared against selected theoretical models of design process to enable an response to the research question which assesses what is observed first hand against what is predicted by some. An exhaustive comparison of directly observed difficulties and the numerous theoretical models is outside the scope of this thesis.

Direct observation of actions within a case study is the pragmatic way to account for tacit knowledge within design processes. There are two ways of achieving such accounts; qualitative accounts which simplify the process and rely on experience and opinion, and an exhaustive assessment combining qualitative and quantitative of design moves within a reflective practice (such as Linkography).

Qualitative accounts alongside graphical documentation, highlighting key moments and complications have been selected for this research over Linkographic methods. The discussion in this section has highlighted the need to select appropriate cases with minimal ambiguity; and with clear and coherent purpose.

\subsection{Document Outline}

In the following section (2) a participant observer case study is used to highlight observable difficulties and issues revealed by the application of explicit DPDT's to a well structured design pattern and problem. In the section following this (3) a case study of architecture students working and thinking through the use of lighting simulation design aids within an iterative design process highlights further issues with the use of generalised DPDT's. Following this, the final section (4) analyses the issues and assesses the hypothesis. 
The two sections which follow are designed in conference paper format. Section two is based around a case study which is a specific parametric modelling project that followed a wider investigation into representation and recreation of the Acropolis Mount and Parthenon; Educational Games: An interactive visual interpretation of the Acropolis and the Parthenon from antiquity to modern times (Mihova and Fraser 200x, 2010). Section three is a case study of a second year architectural design paper (ARCI212; Design Integration) which has a focus of integrating digital simulations and calculation into the design process, and an extract has been presented at CAADRIA2011 and published within conference proceedings (Fraser 2011). This structure is described if fig 10.

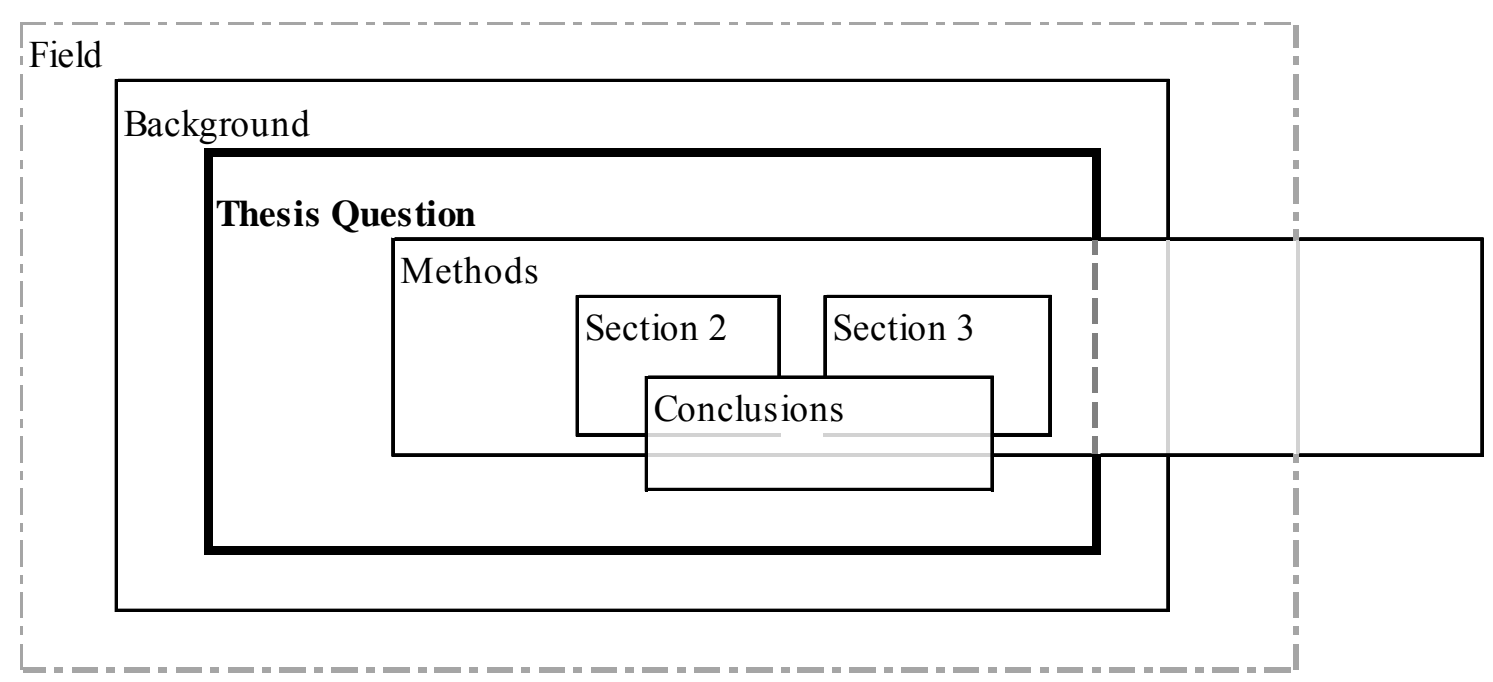

Figure 10. Thesis and document structure 


\subsection{Some obstacles of Modelling Parthenon Elements with Parametric Tools}

This chapter analyses some of the obstructions to the application of digital parametric design tools in architectural problem solving. It demonstrates that loosely structured or explorative uses of explicitly parametric geometric digital tools creates obstructions and 'extra work' which can limit in a very practical manner the solution set available to design practitioners and modellers. It also finds an accommodating analogy and tool metaphor to explain certain difficulties which can be avoided or embraced.

The analysis classifies design decision making strategies within explicitly parametric geometric digital design tools. It explores connections between theoretical approaches in design cognition, architectural modelling, and statistical modelling and uses them to develop an understanding of the limitations of parametricised systems in the context of architectural practice. This analysis is based upon a qualitative account of the negotiated issues, obstructions, and difficulties that arose in the creation of a parametric model of an element of the Athenian Acropolis; Hekatompedon Parthenon as an educational and historical architectural information model and associated artefacts.

This analysis also tests the supposition that strong and recognizable patterns allow greater insight into the obstacles which face parametric modellers than more novel or unusual ones. It finds whilst addressing different inputs and outputs of a dynamic system, consideration of the distinction between proposed categories of obstructions can be beneficial. This benefit is heavily dependent on the contextual requirements including a priori knowledge of the given problem. It is also found to be increased when wicked design problems are constrained to a manageable level of complexity or by following robust patterns.

\subsection{Introduction}

Parametric design is becoming more well-known ${ }^{53}$ and lauded within $\operatorname{architecture}^{54}$. The available tools are seen as increasingly more relevant to architectural design. The increasingly high-powered and high capacity tools are offering more and more modelling options and the ability to compute complex structures of information. The ability to handle

\footnotetext{
${ }^{53}$ Parametric design: through programming methods Burry (2011), elements of Woodbury (2010), processes Sakamoto et al. (2008), Kolarevic (2003), also parametric design workshops in major conferences such as ACADIA, CAADFutures, Smart Geometry amongst others.

${ }_{54}$ Generally Oxman (2010), general advances in technology and parametrics Schumacher (2011), parametric tools Aish (2009), parametric thinking Karle and Kelly (2011).
} 
complexity, previously viewed as a major limitation to the application of the parametric approach, seems to have been largely removed. Now, it seems that if one can conceive a system or configuration, one can find a method within existing programs, or create one's own program from scratch to achieve the same goal, often achieved through programming methods. While complexity is no longer a limiting factor in the majority of cases we might now use it for, the new found ability to calculate means that other factors and obstacles now become more apparent.

The literature in the field of computational design seems generally positive towards the use of digital parametric design tools (DPDT's) ${ }^{55}$. However, little research has focused on the difficulties and obstacles which are part and parcel of the process of using these more involved, more precise tools. The problems with parametric design are generally not welldiscussed in literature, although some new research is explicitly addressing some elements ${ }^{56}$ and there is general acceptance that describing architectural geometries through 'control rigs' (Aish 2005) created through application programming interfaces and methods (Burry 2011) is difficult and time consuming. The difficulties and time issues arise from resolving the complex interrelationships of wicked problems (Rittel and Horst 1973) in architecture. While there are many discrete variables describing a building or artefact which a designer/modeller may define easily, defining a model of their interactions is not straightforward. The generally positive attitude persists even though a modeller typically has to rebuild models multiple times as the often abstract or ill-defined problem is further articulated in a process Woodbury (2010) appropriately calls Edit/Repair/Replace.

In the process of computing geometry in architectural design there is an iterative process of learning how best to find solutions by rearranging the relationships and modifying algorithms. Because architectural problems (and indeed, many of the smaller sub-problems) are wicked in the sense they are multidimensional ${ }^{57}$, there is often no 'best' or 'optimum' solution. There are solutions which range from robust to fragile which a designer/modeller may come across, and likewise there are stronger and weaker methods for identifying these. In tackling this issue, relatively little research has been found addressing the problems of finding solutions through parametric methods even though the field has experienced a

\footnotetext{
${ }^{55}$ Case study examples Hernandez (2006), Theory; Oxman (ibid), Terzidis (2004)

${ }^{56}$ Davis et al (2011a, 2011b) is one example. Woodbury's documentation of design patterns is another example, although it seeks to better understand problems as a way of limiting the difficulties of implementation.

${ }^{57}$ n-dimensional Mahdavi 2002
} 
substantial increase of publications and case studies ${ }^{58}$. This chapter examines some of the issues revealed through the use of DPDT's by looking at a well-documented classical pattern which is both illustrious and for the most part highly rational. It has been selected for review in this thesis because it starts with a design where the underlying relationships which are to be 'programmed' into the parametric system are apparently very well documented, so the focus can be on the model building not the parameterization of the design.

\subsubsection{Case study Considerations}

In order to distinguish difficulties and obstructions in a design process case study, a systematic set of independently derived assessment criteria were developed. The goal was to reduce the complexity of the analysis. A review of cases in the practical literature found a body of cases which were either complicated or vague due to their focus on problem solving methods without presenting or capably illustrating the associated obstacles.

The goal of this case study was also to step the analysis away from the prevalent danger of single issue focus resulting from the tendency in case study methods to identify elegant descriptions of problem / solution pairs within architecture. This tendency produces case studies which are either simplistic in the illustration of the issues or are so intricate that detailed descriptions of design decision making are not practical or are confusing. Drawing general conclusions from these cases is problematic. In an effort to simplify this 'messiness' the following ideal criteria for the selection of a case have been developed:

- The level of complexity should be high enough to allow for testing of the ability of the software to cope with moderate to high level of descriptive complexity ${ }^{59}$. One reason for using a complex example is to examine whether the software can handle complex dependency chains which are not straightforward to model. Alexander's idea of misfit variable is a relevant to this point; where a conflict between form and context variables creates a challenge for design to address (as the 'misfits'), the conflict between form (complexity) and context (computer program) means that there is a worthwhile design challenge.

- That most of the analysis and measurements relates to physical examples (and patterns) is beneficial because there are numerous well-referenced benchmarks that a modeller can consider. The older and better known the example or pattern, the more mature the benchmark information, and hence the parameter relationships can be considered to identify consensus

\footnotetext{
${ }^{58}$ Bhatt et al. (2008, 2009), Meuller (2009b) Martens (2004)

${ }^{59}$ A more precise term for this is Kolmogorov Complexity (K-complexity) see Li and Vitanyi (1997)
} 
from literature. Relating the case to a mature literature featuring numerous suppositions and refutations reduces potential bias in the analysis.

- Rational Geometry allows integration within the media of advanced tools and the language of base principle geometry. An example comprising Michaelangelos's David would be a difficult form to parameterize (if not impossible), while one of Palladio's many Villas would be easier due to the degree of geometric rationalisation.

- Well-documented heuristics and a well-documented logical (hierarchical) order for the parametrisation means that logic chains are readily programmable with heuristic solutions.

- Something is offered by using an advanced tool that traditional methods do not. The case study seeks to identify whether the tool offers the ability to integrate with traditional modes of practice, but adds some further modelling capability.

- An ageless architectural analysis issue which is well referenced in education and straightforward to communicate is far easier to draw general lessons from because these lessons have been well-documented in the theoretical and educational literature.

Such a case fits within a reflective design process. Approaching it as a reflective practitioner (Schon 1983) allows for comparison and assessment of relevant theoretical approaches in design. This case uses Gero's FBS ontology model and Aish's view of associative design as a basis for the documentation of the Schon-like reflection-in-action.

\subsubsection{Background of Parthenon Columns project}

This case study examines a part of a wider, long-term research project Educational Games which produced interactive visual and tactile interpretations of the Acropolis and Parthenon from antiquity to modern times. The project was initiated by Jeni Mihova at Victoria University of Wellington. It progressed in conjunction and conversation with YSMA (Acropolis Restoration Service) to produce prototypes of educational models in a sequence of scales and across several historical ages. Detailed descriptions of this previous work have been published by Mihova and Fraser (2008, 2011). 


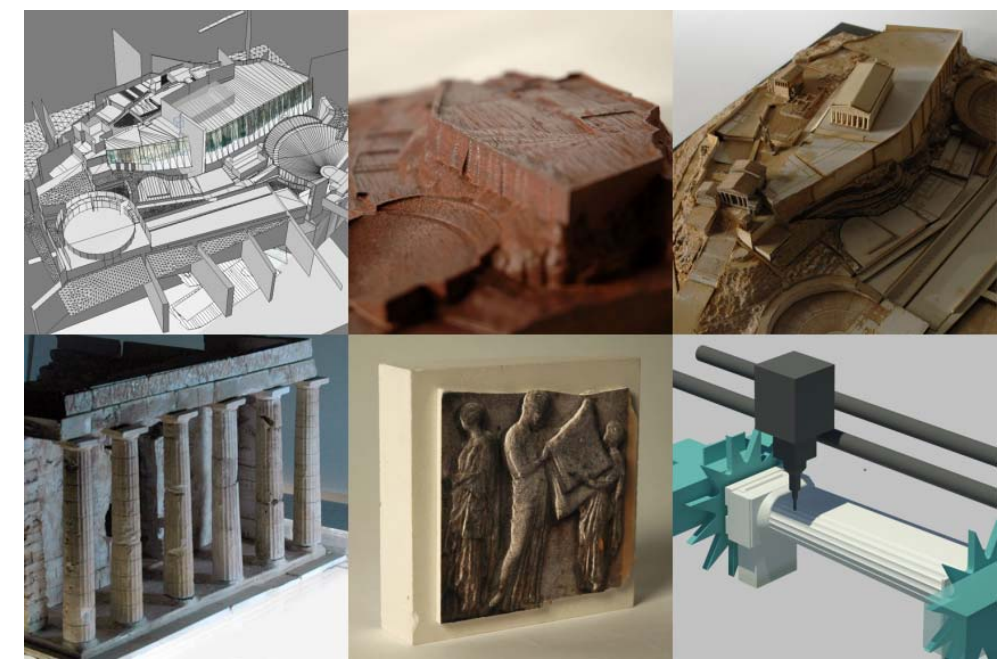

Figure 11. Experimentation prototypes for Educational Games project from large to small scale.

(a) Digital model, (b) 1:2000 landscape, (c) 1:800 Landscape with buildings, (d) 1:40 Cella with Metopes, (e) 1:10 Metopes, (d) Column manufacturing development model.

An early experimental phase of this previous research sought to identify relevant modelling techniques and interpretation methods through digital modelling, $\mathrm{CNC}$, and hand modelling techniques. This presented a variety of possibilities and avenues of exploration. The result was a collection of models ranging from 1:5000 (Acropolis and landscape) to 1:1 (Parthenon Metopes), but excluded some opportunities for digitally modelled rapid prototype artefacts (ibid) due to complexity, budget and resource restrictions (fig 11.). The completed project was exhibited as Masks of Time at Wellington City Library and Victoria University School of Architecture in March/ April 2010. The potential to reinterpret such a well known example of Architecture though updated methods and technologies was one of the central points of focus. A highly relevant example and precedent of visual research into the Parthenon by Debevec $(2004,2005)$ uses newly available technology to map and present models in a new way to a new audience. Other examples of recent research into Doric temples with a focus on technology include Psycharis et al (2000), and Ullrich (2007), and parametric variation in columns Hernandez (2006). All analyse well-defined and well-known patterns in order to extend existing knowledge.

This case study is a reflective analysis focussing on one particular aspect of this exercise which could be applied parametrically to the modelling processes: that of the optical refinements of the perimeter columns and facades through heuristic and associative geometric methods. 
The use of Parthenon column refinements as the basis for a parametric case study meets most of the criteria for examination of reflective modelling process. The pattern of the Parthenon is relatively unambiguous due to the strong geometric rationalisation and published material describing how ancient temples were conceived and executed ${ }^{60}$. Published material and measurements are also widely available. Whilst both the geometric and descriptive complexities ${ }^{61}$ of the project are sufficiently high for there to be some benefit in analysis, the columns do not exist in isolation; the contextual, interpretive, and artistic features must be acknowledged and thus integrated with architectural traditions. The use of technology is present in affording the wider appreciation of this well known example to provide new interpretations. No explicitly parametric models of the Parthenon columns and lower facades are known to exist at this time and so it is a unique investigation.

\subsection{Overview of case}

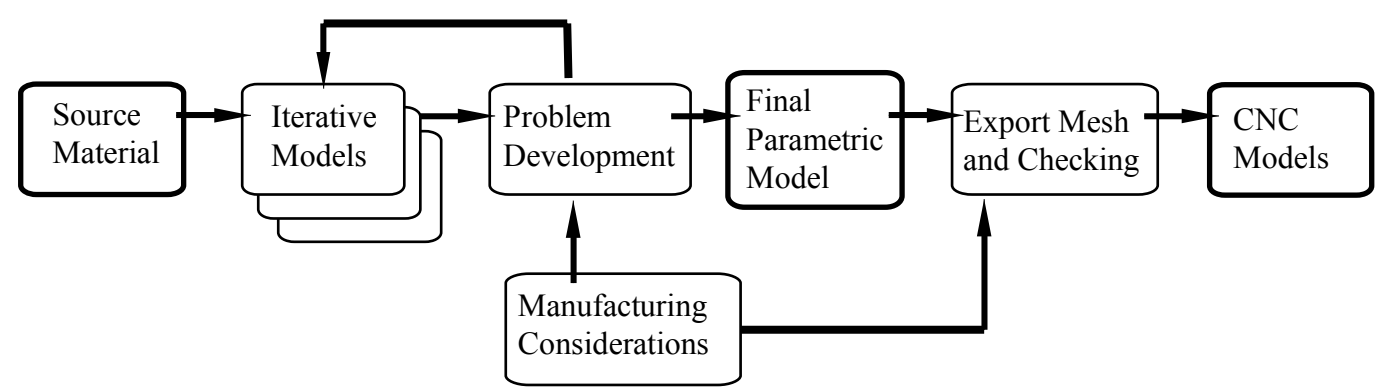

Figure 12. Project workflow from source material to physical artefact

The Parthenon Columns project sought to produce CNC rapid prototype models of a portion of the Parthenon columns and related geometry to illustrate both the observable optical refinements and the design thinking behind the complex hierarchy of dependencies therein. fig 12 provides an overview of the processes. Multiple and diverse sources were collected and used to analyse the relationships at play and the heuristics extracted or appropriate methods substituted. A series of conceptual parametric models were built within GenerativeComponents (Aish2006) which iteratively assembled and assessed control rigs against the source material. The modelling scope, problem definition, and problem decomposition were refined to produce a final parametric model of a portion of the Eastern façade and Cella columns in the form of geometric solids. Two contrasting instances from this model were extracted from the final parametric model to illustrate the position of the

\footnotetext{
${ }^{60}$ See Virtuvius Pollio, De architectura, Book 4 Chapters 3 and 4. See also; notes 63-65

${ }^{61}$ See note 58
} 
refinements within the hierarchy of logic within the design: one accurately represents the actual configuration; and the other exaggerates the effects in order to make them more visible. The models were exported as meshes, checked, and manufactured through CNC rapid prototyping in plastic / resin materials. The final product is a pair of sectioned models at 1:150 scale. The stages in the process are illustrated in fig 13.
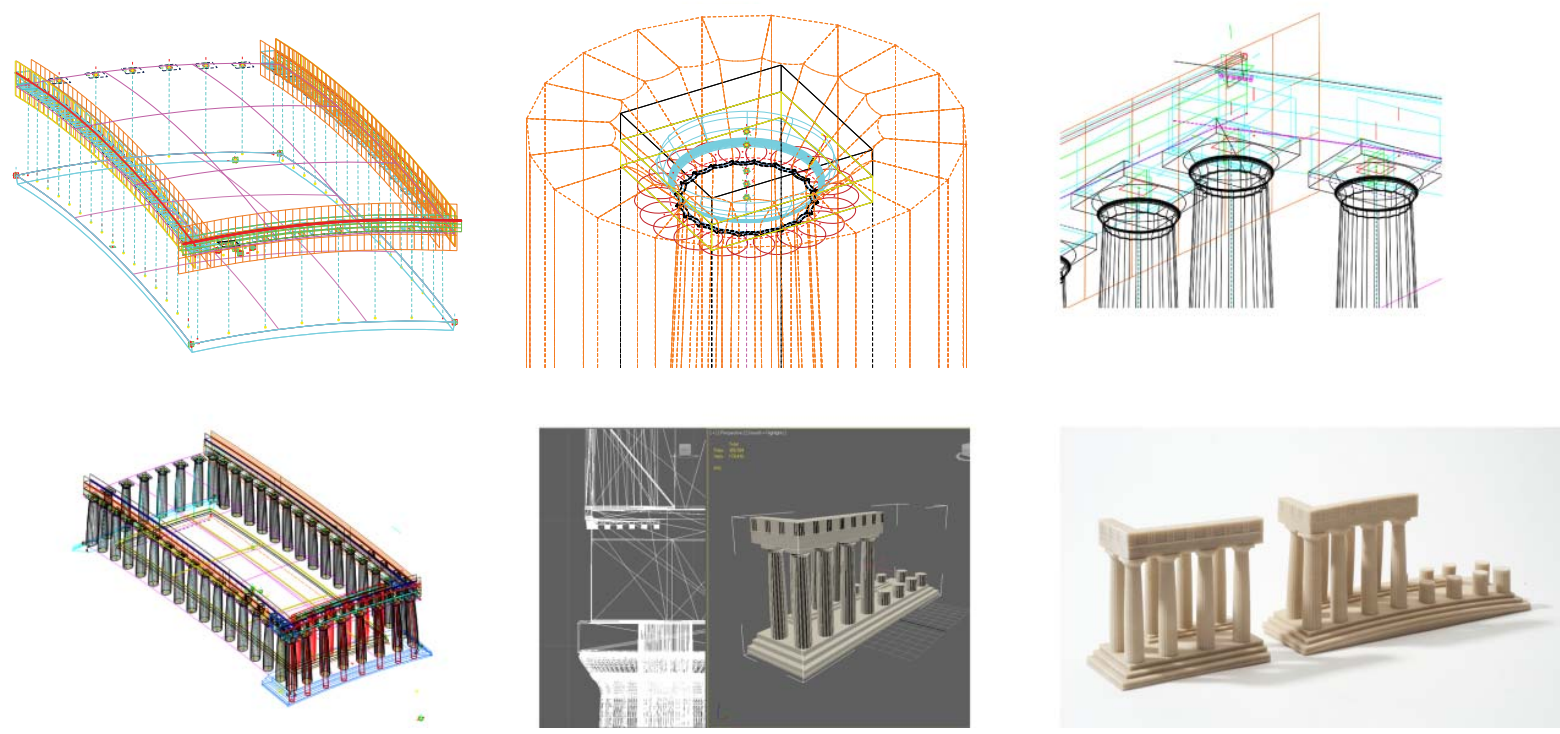

Figure 13. Project overview; Development of source material into multiple iterative models (a, b, c), final parametric model (d), export as mesh and checking (e), 1:150 CNC prototypes (f)

\subsubsection{Source Material}

At the beginning of the modelling process an extensive set of source material was identified and collected. This consisted of drawings ${ }^{62}$, theories on the Parthenon proportions ${ }^{63}$, historical accounts ${ }^{64}$. Multiple other sources were referred to including accounts of Entasis, Stylobase curvature, and column incline ${ }^{65}$ as well as modern day photographs of Parthenon monuments $^{66}$. Before any modelling within GenerativeComponents (GC) began the collected information was categorised by the level of abstraction and relevance to the proposed geometric representation methods. Earlier work (fig x; (d) 1:40 Cella) which featured geometry at the level of individual stones was identified as too high a level of detail because

\footnotetext{
${ }^{62}$ Stuart and Revitt (1762), Korres (1992, 1993, 2000), Angelopoulos 2003, Carrey (1674)

${ }^{63}$ Van Mersbergen (1998), Markowsky (1992)

${ }^{64}$ Penrose (1888), Zambas (2002), Hurwit (2000, 2005), definitions of Doric temples Vitruvius Pollio (15BC)

${ }^{65}$ Tansey and Kleiner (1996), Dismoor (1902), Coulton (1977), see also fig x.

${ }^{66}$ Image search term (Google) "Parthenon" list many photographs of any and all surviving elements, including multiple angles and details.
} 
the nature of the geometric refinements results in a global level of abstraction at a different scale; for example the incline of a column affects the overall form and not just the individual stones in direct contact. The diverse selection of source material was restricted by this early design move. Relevant measured observations which relate to the necessary level of abstraction can be summarised as follows and in fig 14 .

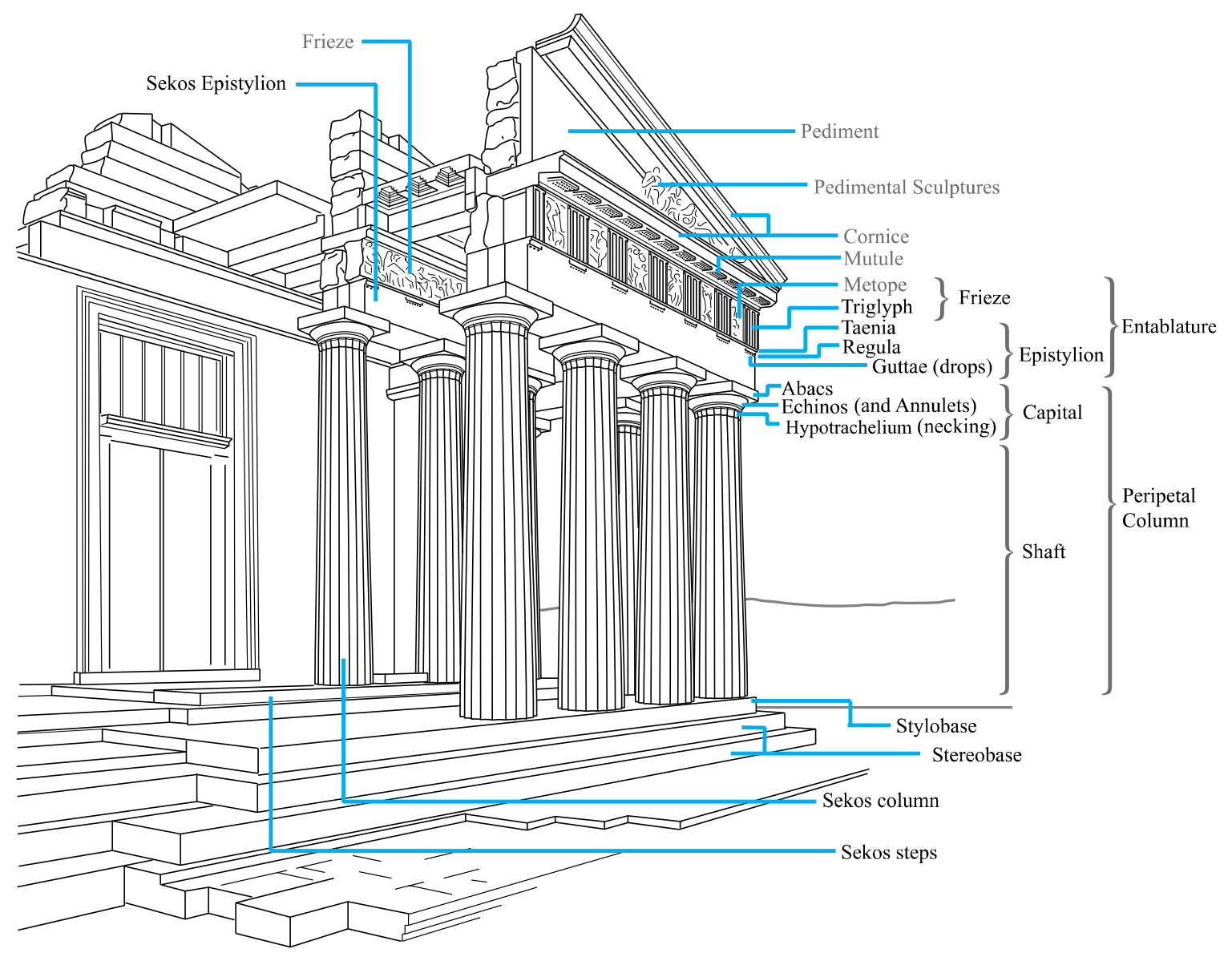

Figure 14. Parthenon anatomy after Neimann ${ }^{67}$

- Edge lengths, width, corner offsets, and curvature of Stylobase and Sterobase

- Column and Entablature vertical inclines

- Column dimensions and variations; positions, circumferences, entasis curves, corner column enlargements, incline of axes, intercolumniations

- Column details; shaft fillets, hypotrachelium ('necking'), Abacs, Echinos, Annulets

- Epistylia (architrave) geometry; Taenia, Regula, Guttae

\footnotetext{
${ }^{67}$ Disposition of the sculptures of the Parthenon In Smith (1908) A guide to the sculptures of the Parthenon in the British Museum
} 
While many of the sources are graphical in nature, there are quite a few also selected for inclusion which describe measurements and reasoning within generalised textual accounts ${ }^{68}$. These are primarily the historical traditions of analysis of ancient architecture, the symbolic nature of measurements and relation to ancient units of measure, and discussions accounting for discrepancies between researcher's measurements or variation between individual columns or blocks. Some of this category of less explicit source material proved generally helpful in understanding how the project was initially conceived and laid out; and more specifically how heuristic methods of abstracting the geometry allows for an understanding of the Parthenon as a pattern which can accommodate variation.

An example is the degree of accuracy of the stones in relation to an idealised model; Penrose (1888) identified that the edges and corners of the base vary in length and relative height ${ }^{69}$ and also proposed that this was not intentional via measurements of cracks accounting for possible movement from original positions. Balanos (1940) repeats the measurements with a higher accuracy and agrees with the Penrose dimensions, but proposes alongside Korres (1993) that these were in fact intentional hyper-refinements to promote asymmetry. Whilst this points in the general direction that the differences in corner elevation are intentional, the evidence is not definitive. Zambas (2002) points out that similar vertical differences occur in comparable

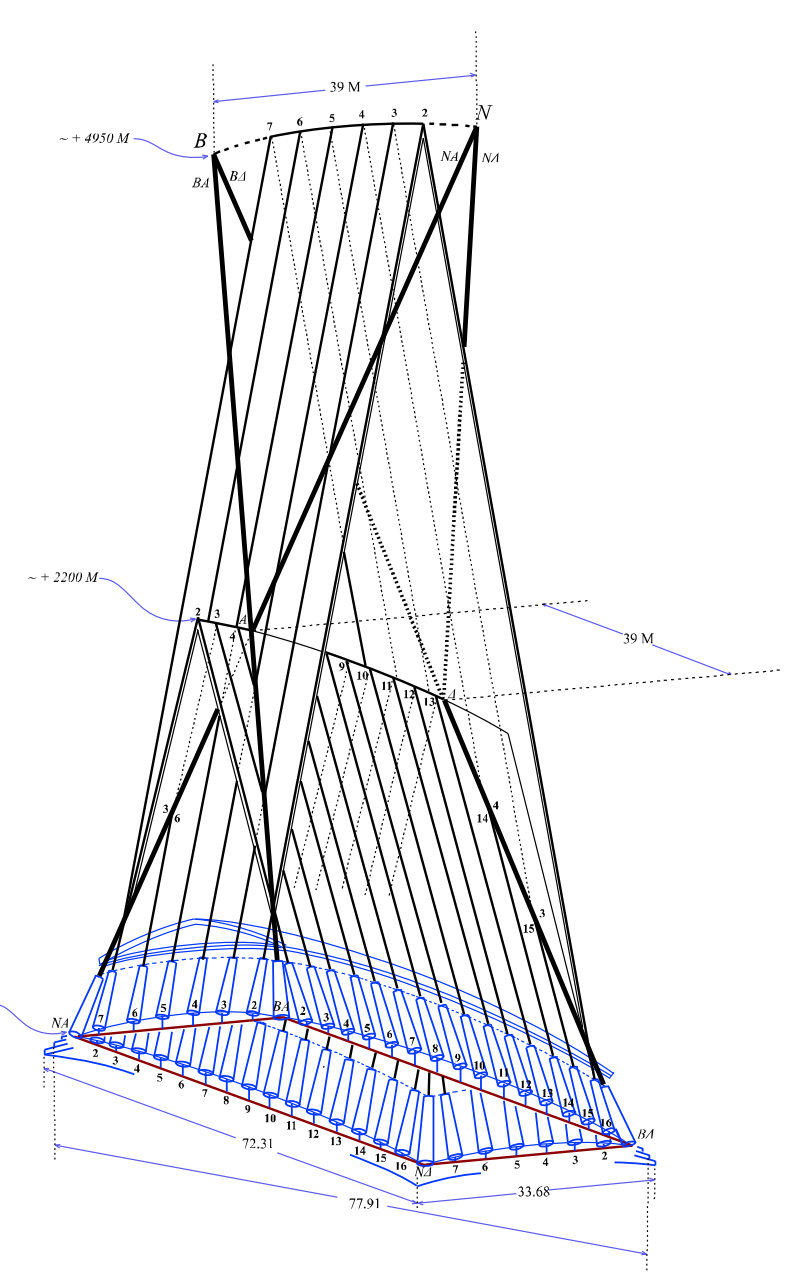

Figure 15. Parthenon hyper refinements after Korres (1993) temples of that time and that ancient levelling techniques provided similar degrees of accuracy $^{70}$. The agreed relative difference in elevation of the corners (within accuracy limits)

\footnotetext{
${ }^{68}$ Dinsmoor (1902), Korres (1993), Balanos (1940).

${ }^{69}$ See red oulines in fig 15.; Stylobase corners are represented by relative elevation and incline of these lines

${ }^{70}$ Also suggested by several authors is the effect of the Morosini Explosion in 1687 during the Venetian siege had on the foundations.
} 
is very close to $29 \mathrm{~mm}^{71}$. Whilst the figure can be expressed as a variable within the proposed parametric model, the relationship between the Stylobase geometry and the columns and Epistylion means that the finer points of how the geometric generators are operating is fundamental. To model such a relationship means accounting for column geometry as dependent on the Stylobase. A highly relevant example of an illustrative diagram of the Parthenon and the refinements in the base is Korres (1993) drawing illustrating Stylobase and Entablature curvature as well as column incline which forms the basis for the parametric model described in this case study (fig 15). Kleiner, Dinsmoor, and Coulton are also helpful in illustrating the particular refinements of base curvature transfer to upper elements, column incline and corner enlargement (fig 16).
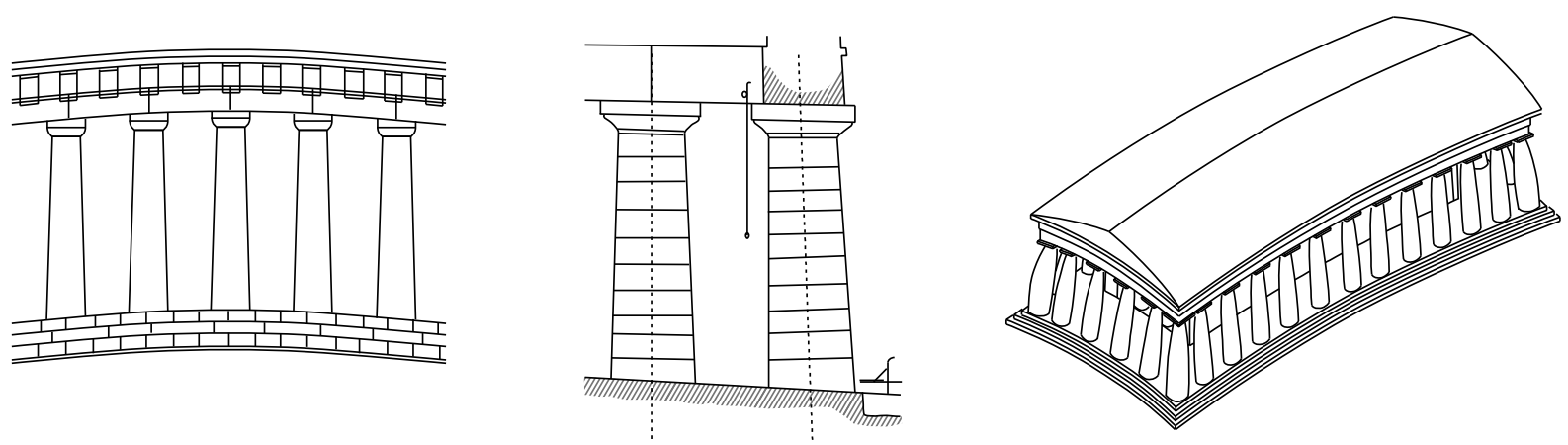

Figure 16. Hyper refinements of Greek temples;

(a) after Tansley and Kleiner (1996): transfer of Stylobase curve to Epistyle,

(b) after Dinsmoor (1902): Column axis incline on curved Stylobase,

(c) after Coulton (1977): Reconstruction of Greek temple showing exaggerated curvature employed by builders (Stylobase curvature transferred to upper elements, column incline, and corner column enlargement)

It can be further noted from the collection of sources that the dependencies of an idealised Parthenon occur in a relatively straightforward 'anatomical' manner; from the ground up, but that the refinements which the structure is famous (fig 16) for have a subtle effect on the overall form. Without the generalised accounts ranging over a large time frame these subtle effects and refinements could easily be discounted due to issues of built accuracy, movement, or aesthetics. Collecting of source material for modelling did not occur as a discrete step, but involved a large initial collection and was supplemented following problem development.

\footnotetext{
${ }^{71}$ Angelopoulos (2003) indicates that the SE corner has a relative elevation of $28.9 \mathrm{~mm}$ above the SW corner, and the NW corner is elevated $1.8 \mathrm{~mm}$ above the SW.
} 


\subsubsection{Iterative Models and Problem Development}

Following analysis of the initial sources, a series of parametric models was created within GC. The first set of models consisted of a generalised way of modelling the base of the Parthenon, with the intention of iteratively working 'up' the structure. The model evolved quickly from a flat slab to a curved slab (torus patch geometry) to a model with greater degrees of flexibility (fig 18); so that the relative heights of the base corners and length of edges addressed in literature could be accounted for. This early stage can be characterised as relatively straightforward, but critical in anticipating and setting up the downstream dependencies of column axes. While the effect of a model with degrees of flexibility is, for all practical purposes, accurate, one reported aspect of Greek methods for curve creation was not emulated; diminution. Diminution is a heuristic method for approximating a curve used by Greek architects (and ever since these times) and masons to set out dimensions which were too large for compasses or jigs. It subdivides two dimensions of a plane and creates a fine grained grid in which points are plotted to correlate approximately with the proportions of parallel chords within a equally divided circle. The meaning of the word in regard to geometry is 'to decrease' or 'lessening'; and it is the incremental reduction of the difference between regular divisions which is reduced by a preordained proportion or pattern. The parametric model in this case study uses arcs to approximate the curve created by periodic subdivision of lengths and proportions of displacement as in the application of the ancient method of diminution (fig 17.).

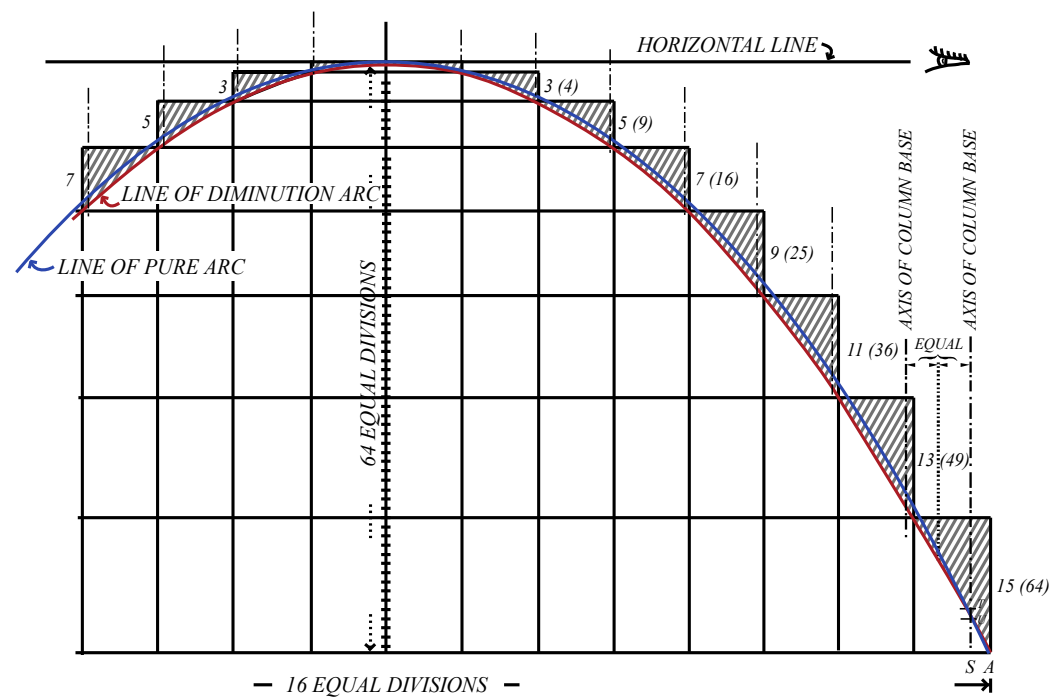

Figure 17. Diminution method in Greek temple Stylobase after Dinsmoor (1902).

Curve of diminution (red), Curve of true arc (blue) 
The practical limits of the available tool in this instance assisted in defining the initial problem at this stage; that approximations, or heuristics, are a necessary time saver and that despite causing inaccuracies were necessary to help understand how to create a model with complex interrelationships. The diminution method is in itself a heuristic methodology for building an approximated arc. The logic behind using relevant yet somewhat inaccurate modelling methods in the parametric model was that future developments could re-write more accurate definitions of geometry if and when required. A comparison of modelling methods was core in making a design decision which affected absolute accuracy which had to be balanced with efficiencies of modelling and computation. Following this comparison a design decision was made to substitute arcs and B-Splines throughout the study for the column shaft fillets and Entasis, the Enchion and Annulets on the column capitals, as well as the Stylobates described in figs 17 and 18. This method substitutes methods readily available in current modelling tools in place of diminution methods which were available and convenient to the architects and stonemasons of during the original construction of the Parthenon.

\section{The Base}

Stylobase set-out and development followed the following steps. A simple rectangle was speedily developed into a variable trapezoid and a three dimensional set of lines which linked the corners of the base and allowed variation in heights. This is in line with the reports and debate regarding the relative elevations and edge curves outlined by by Penrose, Balanos, Korres, and Zambas. Arcs in the vertical plane were generated from this form on all four edges and created a surface which represented the doubly-curved Stylobase (fig 18.). This modelling stage was relatively straightforward, but took several iterations to find a solution which could accommodate variation and exaggeration. This act of anticipating the future development of the parametric model is a clear example of anticipation of degrees of freedom'. Few 'dead end' methods were encountered in this stage, and the interface (the unconstrained variables which a user can modify) was arranged in a logical and uncomplicated manner relatively easily. 

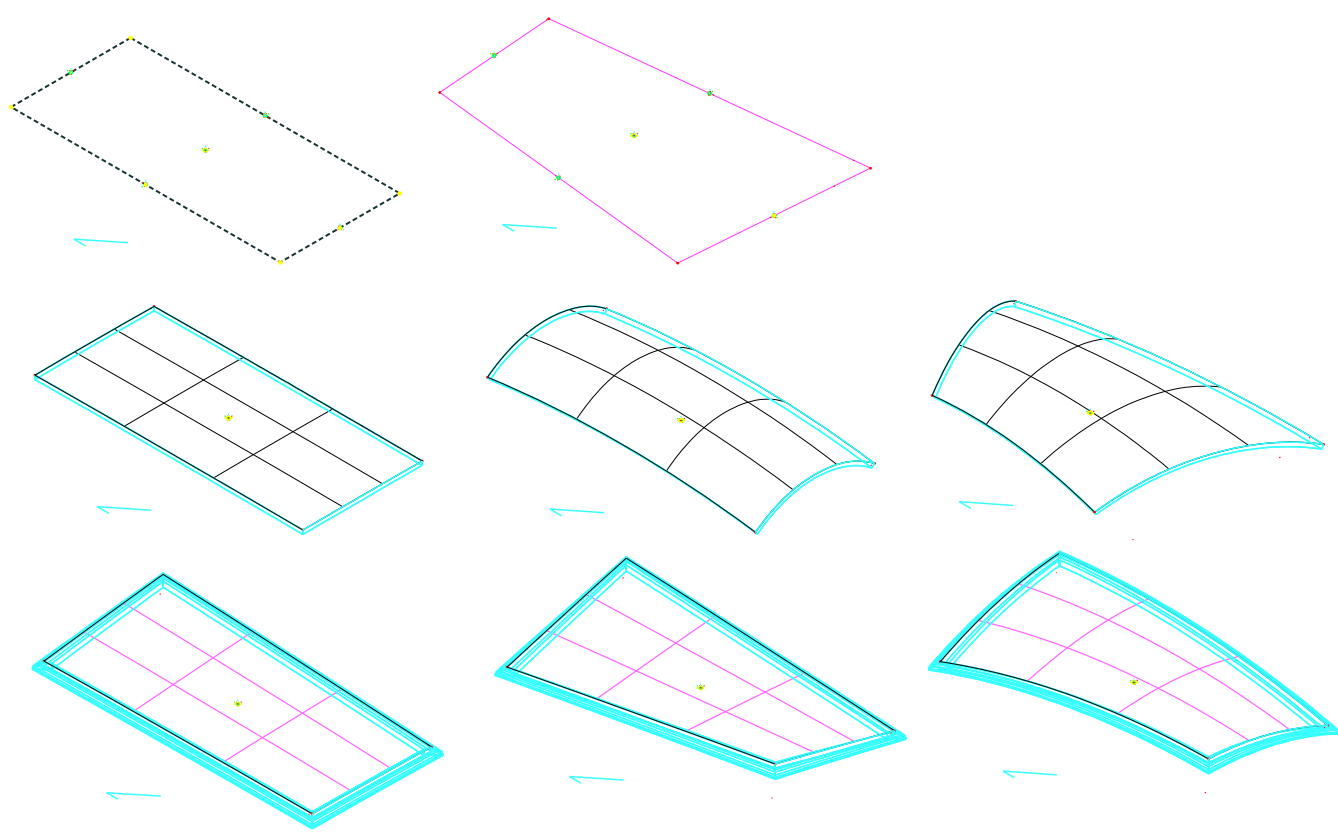

Figure 18. Stylobase setout and flexibility. Number of model iterations $=5$, Number of variables $\approx 15$

The next group of models investigated and modelled the set-out of column axes and inclines. Several factors were considered and parameterised; offset from base edge, corner and normal intercolumnal distances and factors based on column circumference, inwards inclines for both NS and WE facades (by convergence distance as in fig 15.), and column height. The Korres sketch (fig 15.) was influential at this stage but also caused a 'dead end' when translating the previous modelling stage which set out degrees of flexibility of the base.
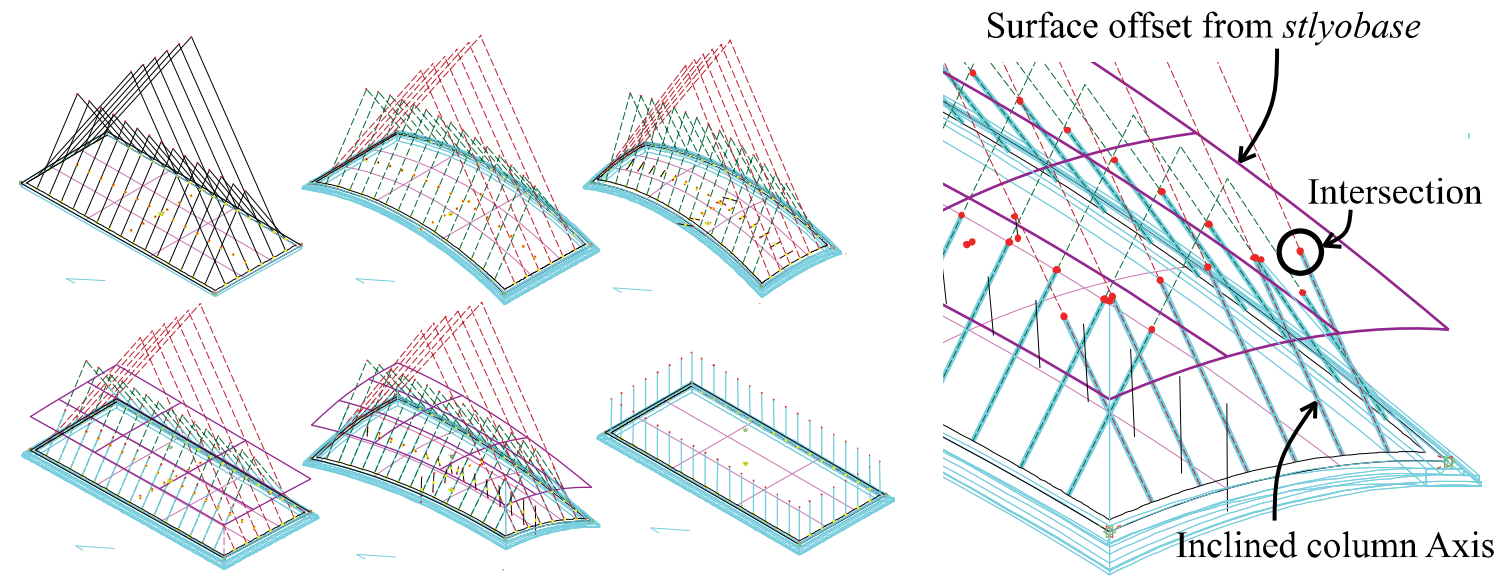

Figure 19. Column axis placement and incline and degrees of freedom.

Approximation of observed column incline with control rig hidden lower middle. Enlargement of column termination heuristic right. Number of model iterations $=4$ Number of variables $\approx 25$ 
Immediately noticeable was the conflict between standardised measurements of column heights and the potential for incline variation; on the Parthenon itself the inclines are minor $(1 / 150)$ and so this discrepancy is not noticeable and within tolerances, but within a parametric model which can accommodate extreme values (fig 19) columns of constant axis length do not terminate at the same elevation when inclined. For this reason a geometric solution was found; the Stylobase surface was offset vertically as an axis termination heuristic to which the different axes intersect (fig. 19 (far right)). This method maintains the effect of column termination at a fixed height above the base in an uncomplicated manner, and allows for independent variables of façade inclines and column termini rather than dependent ones. This design move was essential in maintaining the flexibility of the model, although clearly is not the method which was used by the Parthenon architects and masons. The final model in this set required four iterations, some of which changed the morphology and required a near-rebuild of this stage as were the arrangement of variables which allow user modification and exaggeration.

\section{The Columns}

The columns were treated from the outset as subobjects or subroutines and modelled separately, then populated into the 'global model'. This meant that the circumference and height of columns were created as independent variables; rather than being created as dependent on global variables such as façade width and overall building proportions. On looking into the finer details of the geometry of the columns it became apparent that considerations of manufacture needed to be made. The type of output required from a geometric model to be manufactured through the popular fused layer deposition (FLD) rapid prototyping needs to be

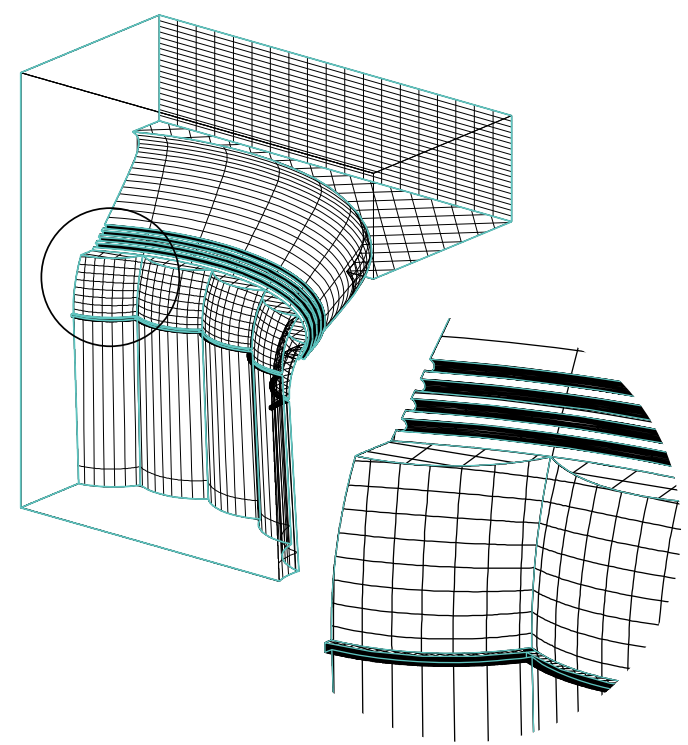

Figure 20. Illustration of problematic individual surfaces method of modelling a column. (Pre-meshing control surface shown) rational, manifold, and 'watertight' (no holes). Typically this is achieved through careful modelling of meshes which meet these requirements and are encoded as Stereolithography (STL) files. The problem with creating surfaces of Parthenon columns which meet perfectly 
and enclose a solid form is that such a method, whilst reasonably computationally efficient, requires so much extra work that the method is not practicable.

The majority of the surrounding modelling elements were straightforward, and easily described with curved surfaces, but the column with 96 trimmed surfaces which meet precisely caused a complex problem in that when exported as a mesh (triangles) tiny holes were created which prevent digital manufacture (fig 20). An alternative method of constructive solid modelling (akin to additive and subtractive sculpting) was identified as a viable alternative, and has obvious parallels with ancient sculpting techniques. Fig 21 illustrates several models which used this technique in a parameterised manner.

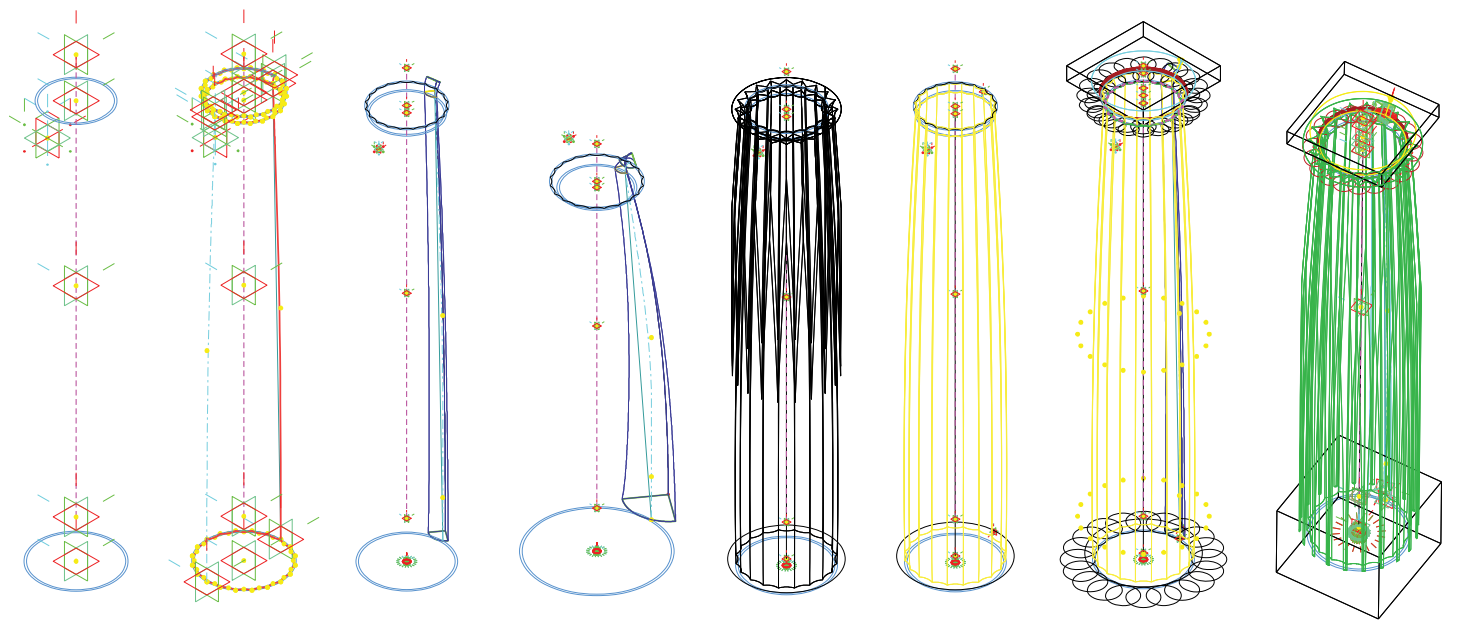

Figure 21. Column first iteration set (a-h). Set-out (a,b), parametricised 'solid' element modelling (c) and freedom (d), rough 'boolean' solid operation with unwanted artefacts (e-h),
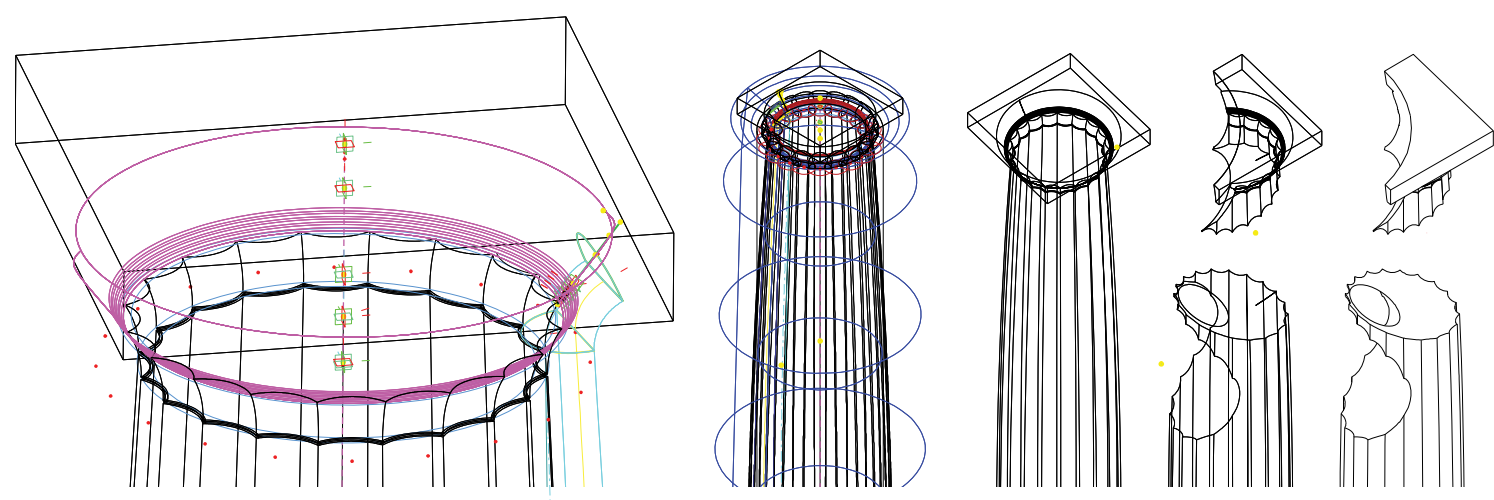

Fig 22. Model from initial solid column. Capital detail (a), 'lathe' operation to remove unwanted artefacts (b), and end result (c); an accurate, but highly inefficient parametricised solid model. 15 model iterations and number of variables $\approx 20$ 
The strategy of individual and unique columns produced a global model which was large and unwieldy when all 46 columns were generated through individual axes and a shared base surface. Limitations with the strategy of solid modelling became clear; the number of unique elements was placing a huge computational load on the processor and the time taken for the model to rebuild itself after a change was around 10-15 minutes. Upon finding a practical limit to the level of complexity the scope and purpose of the model (or the problem definition) needed to be questioned. The entire project definition was reconfigured as a result of this finding and limited to the following conditions:

- Column incline and Stylobase curvature was prioritised over corner displacement; as these two features hold a greater significance to the project as a whole and are more easily illustrated. The Stylobase curvature is a prominent topic of analysis, as is evidenced by the numerous references and illustrations highlighting this point. The Korres (fig 15), Tansley and Kleiner, Dinsmoor, and Coulton (fig 16 (a,b,c respectively) illustrations all prominently convey this curvature. The vertical offset of the base corners is so minor (being $29 \mathrm{~mm}$ at the most) on the overall form that only a large scope and scale model could convey, and poorly so, the effect. It is also worth noting that there is disagreement on this subject between authors; and potentially, or probably not a concern to the original masons of the Parthenon. The degree of freedom of base corner height variation introduced at the first modelling stage (fig 18) appeared to be not as important or as necessary as the overall requirements of the model changed during this analysis.

- Columns are similar enough at an idealised level for instances (copies) to be used instead of generating each column individually. The parametric model of columns was helpful in creating a modified version for the corner columns; which were conceived to be 50/49 the circumference of the standard size, and also the Cella Columns which are slightly different. The precise modelling of the base of columns meeting a curved surface (fig 23) was not necessary; an intersecting set of solid forms hides such a discrepancy and also means the production method is less prone to errors. The shaped topmost surface of the Abacs on the column capital as described in the Dinsmoor illustration (fig 16 (b)) was omitted from consideration, further reducing the necessity for a 'unique' column method. 

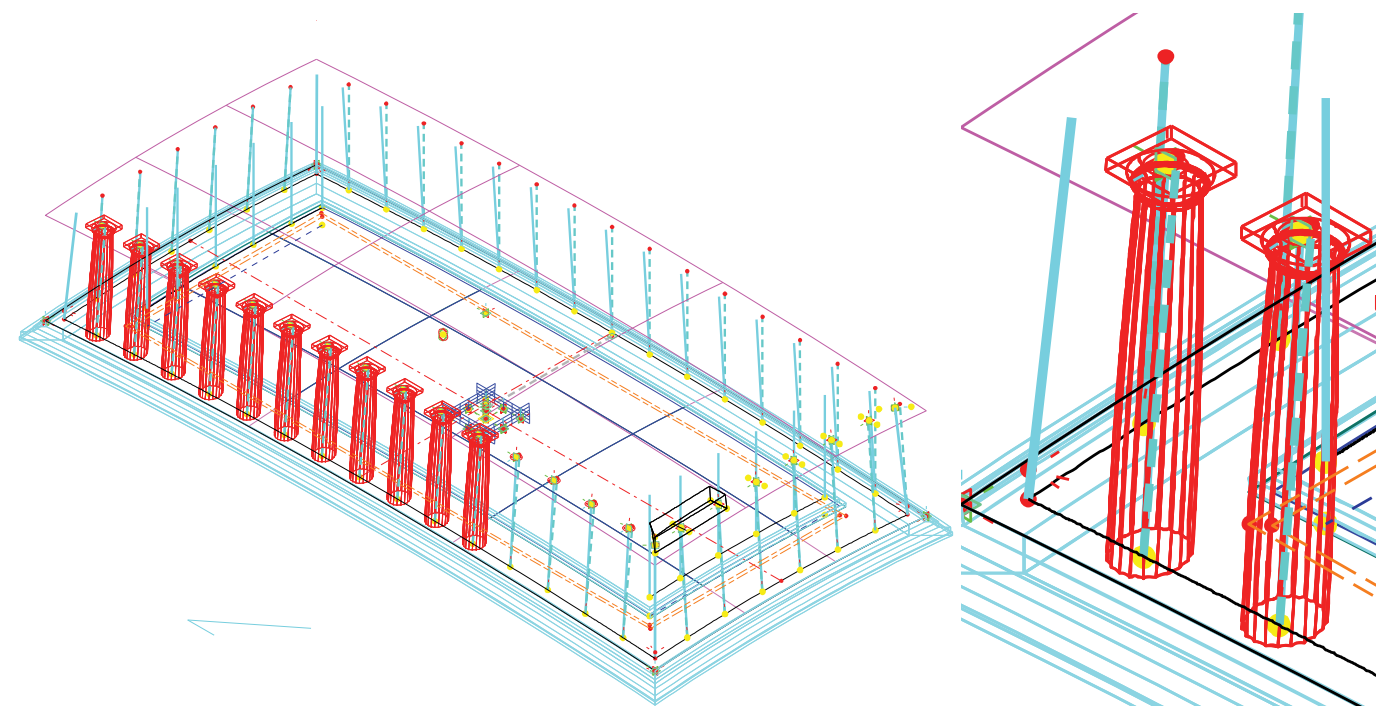

Fig 23. 'Unique column' method; computationally expensive.

(a) Limits of computation approached, (b) precise modelling of column base meeting curved Stylobase.

Calculation time for 12 columns $\approx 15$ minutes

- The Parthenon footprint was 'sliced' to allow a short façade and Cella columns only. This both reduced the computational load and allowed clearer illustration of prioritised refinements. This is in concordance with the historical illustrations in fig $16(b, c)$ which selectively slice the overall form to illustrate specific refinements in geometry.

- The vertical scope was limited to the top of the Frieze (including, but simplifying the peripetal Metope blocks) for two reasons:

- So that the geometric model remained straightforward and did not aim to include freeform sculptural elements such as the Pediment or Metopes, which were clearly futile to model in a parametric manner due to non- rational geometry.

- So that the influence of column incline of the incline of the Epistylia face could be illustrated, as shown in the historical illustration in fig 16(b), and as subsequently modelled in the Epistylion modelling phase; the Epistyle faces modelled as inclined surfaces as shown in fig 25.

Following this analysis and reconfiguration of the design problem the parametric column object was rebuilt from scratch. Familiarity with the geometry and relationships from previous models meant that this rebuild was relatively straightforward and rapid, although not without problems. At first unexplainable, several problems with the software began to emerge; the solid modelling techniques began to work sometimes and other times failed completely, in part due to heavy computational load. Work-around solutions to this problem 
were developed only through developing an understanding of how GC was calculating and representing solid forms before and after addition / subtraction operations ${ }^{72}$. Fig 24 illustrates the modelling sequence for the final column subroutine which solved these problems.
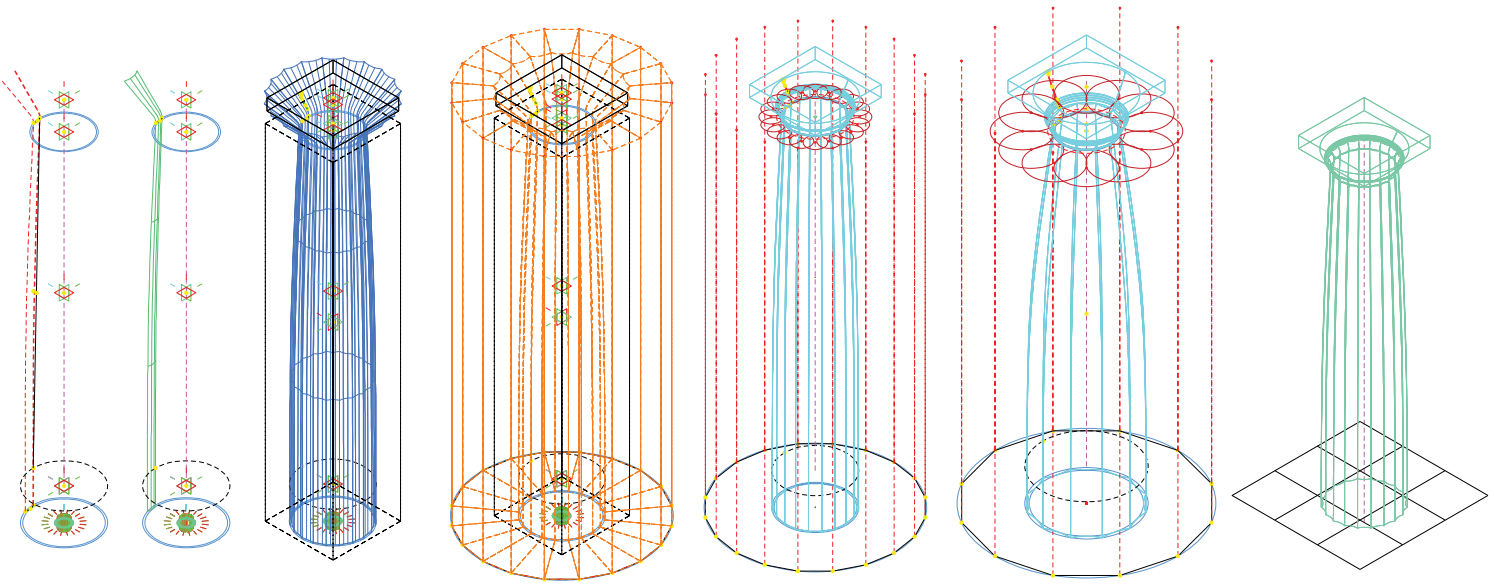

Figure 24. Final Column solid modelling sequence. The final subroutine has been modelled as dependant on a line representing the column axis and a surface which trims the solid geometry (far right).

5 model iterations and number of variables $\approx 20$

\section{The Entablature}

The Epistylia were identified as the last piece of the puzzle after the reconfiguration of problem description and scope. The particular problems in interpreting how these elements, which sit atop the columns, were arranged were split between continuing the influence of Stylobase and column distortions, and the simplification away from individual blocks towards idealised continuous solids. Both had an effect on the modelling strategy which, following several iterations, took the structure of bounding surfaces which could 'cut' the curved faces of the Epistylion and also be offset to allow smaller trim details to align in a dependent manner (fig 25). Triglyphs and Regula were, apart from the corner locations, repetitive and were modelled as separate subroutines and positioned onto the continuous Epistylia. Corner locations were, in particular, difficult because of the need for a solid block with near right angles to locate offset from two curved, inclined surfaces ${ }^{73}$.

Historical sources were lacking in measurement and suppositions on this area of the Parthenon; little could be found to assist in finding a robust method for the corner Triglyphs.

\footnotetext{
${ }^{72}$ A simple explanation to describe the workaround is that GenerativeComponents was 'tricked' into understanding the numerous solid and void constituents in a more convenient manner after encountering problems.

${ }^{73}$ Note corner Triglyph (light blue, far right) in fig 25 is 'split'. Curved, inclined surface in orange.
} 
A simple method of slicing two blocks so they meet approximately was used as the relative importance of this area was not high. A similar problem was encountered when placing a curved Epistylia atop square ended inclined columns. The curve observed in the Parthenon is so minor (curved short façade maximum vertical displacement is widely agreed at $64 \mathrm{~mm}$ ) that the original positioning of individual blocks posed no problem during construction. Upon considering the requirements of the proposed prototyping method another 'work-around' was required; to maintain a series of continuous solids that either meet perfectly or intersect. Extra solids were created to connect the two elements and thus allow for manufacture of a continuous and unbroken solid form.
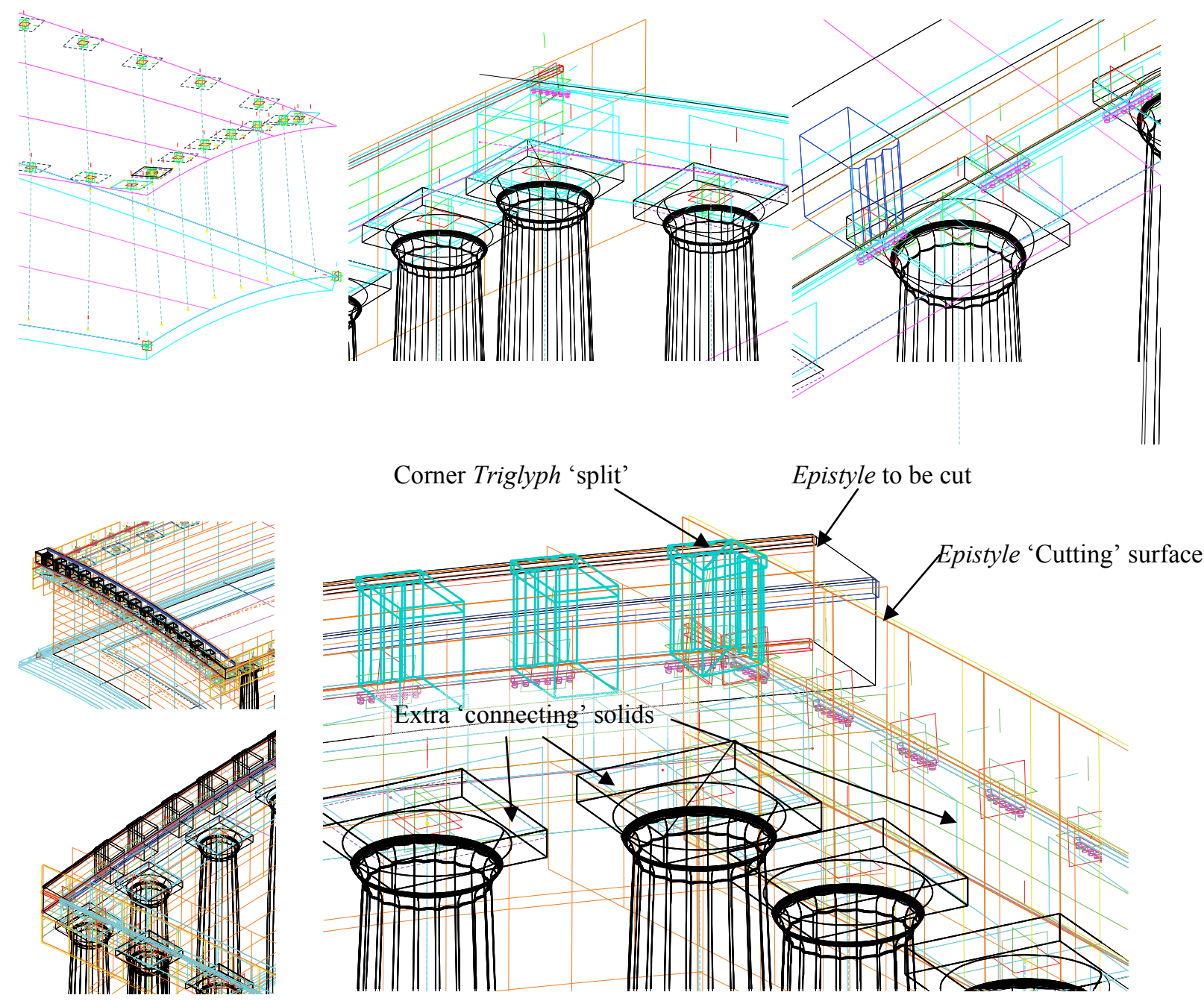

Fig 25. Epistylion modelling stage; note (lower right) corner Triglyph in light blue, 'cutting' surfaces in orange.

The completion of the Epistylia and integration of all included Parthenon elements could be characterised as a cluttered, somewhat messy, slow process. Minor changes, which numbered at least 100 , caused the model to update and this could take several minutes each time. 
Several inefficient work-around methods were used as a compromise between accuracy, time and effort, and requirements of manufacture. These can be understood as heuristic solutions. An unusual aspect of this modelling stage was the realisation that the limit of computability within $G C$ had been reached. The more complex and inter-related the geometry, the more 'bugs' and errors in calculation occurred. This occurred frequently with solids which comprised many smaller sub-objects, and in certain circumstances the model simply 'fell apart' or caused unexplained failures and curiosities (fig 26.)

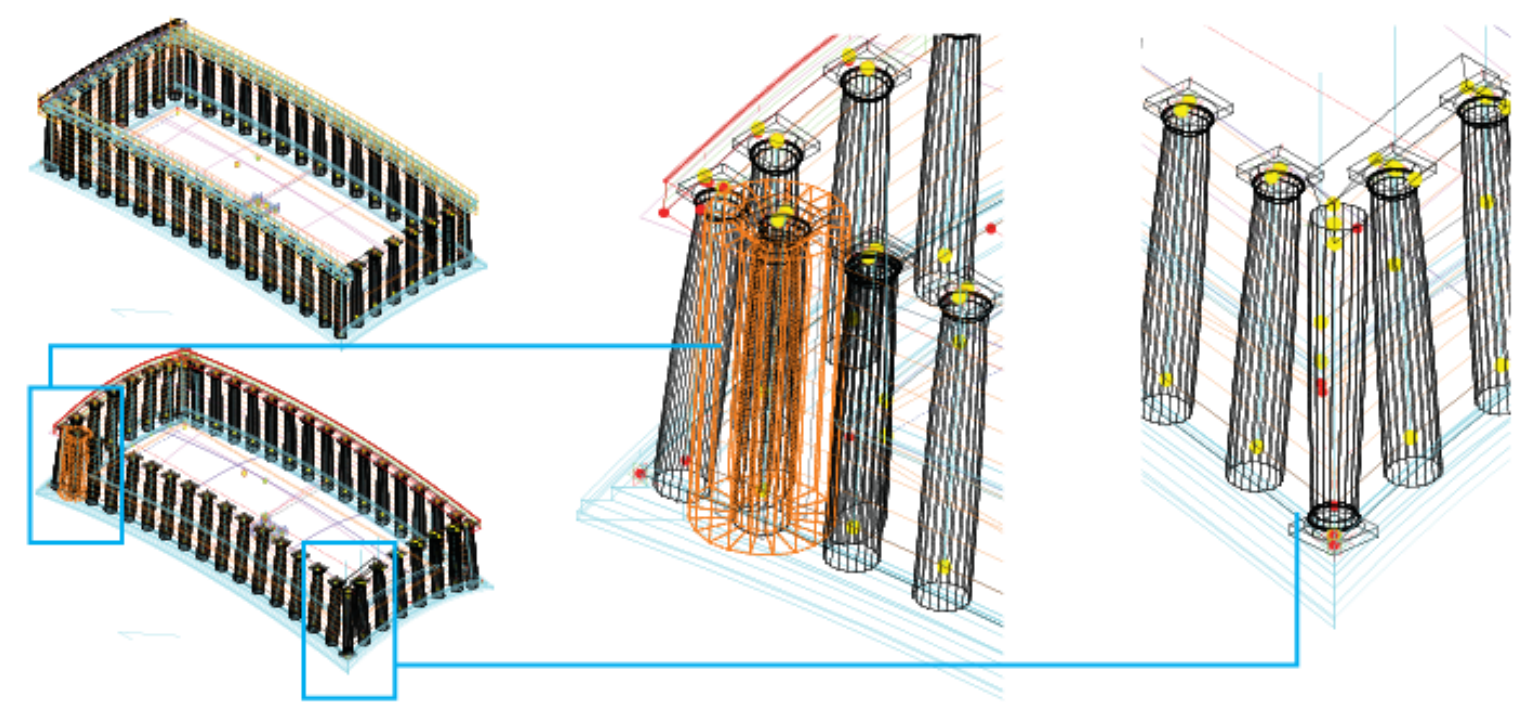

Figure 26. Integrated model (a), workarounds could cause errors and bugs in certain circumstances (b)

\subsubsection{Manufacturing Considerations, Export, and Checking}

Whilst the majority of design moves, reflection, and problem development design moves occurred during the iterative model and development phases, several factors influencing how the outputs were manufactured were taken into account to produce the final parametric model. The most critical factor was the clarification of the design problem to which this model applied; the illustration of selected Parthenon refinements. What became clear during the iterative modelling process was the scope of the model required size constraint which minimised the cost of the model, made clear the observable detail, and illustrated the refinements. The final model (fig 27) was therefore created by taking the integrated model and 'slicing' the short façade to allow constrained scope. Manufacturing considerations such as scale, volume of model solids and cost of prototyping were considered in setting the boundaries of this slice. 

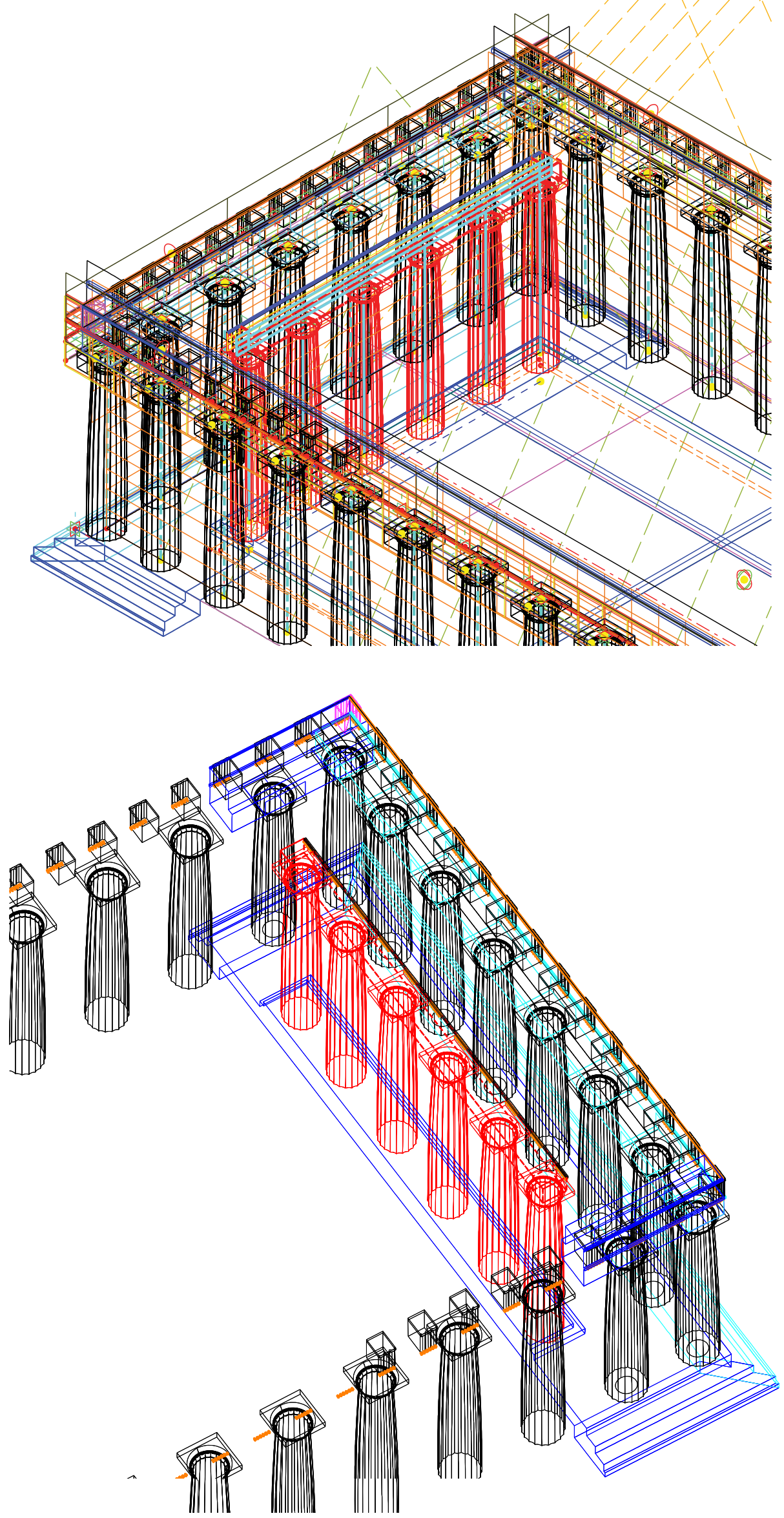

Figure 27. Enlargement of final model with control rigs (a), without (b) 
A scale of 1:150 was selected as a good balance between these different modelling criteria. Further manufacturing considerations were necessary to process and check that the model was 'printable' and fit within the manufacturing 'rules'. Further last minute modelling was used to hollow out the columns and base to reduce the volume and cost of the physically printed objects. Two instances were exported from this model to be prototyped to illustrate a scale recreation of the Parthenon and an exaggerated model similar to the Korres illustration (fig15). The selection of these models was a critical step in the design process. The illustrative aspects and the purpose of the digital model revolve around the ability to represent the hierarchy of dependencies. The two prototyped 1:150 scale models, the detail level of the material, and the curved elements can be observed in fig 28.

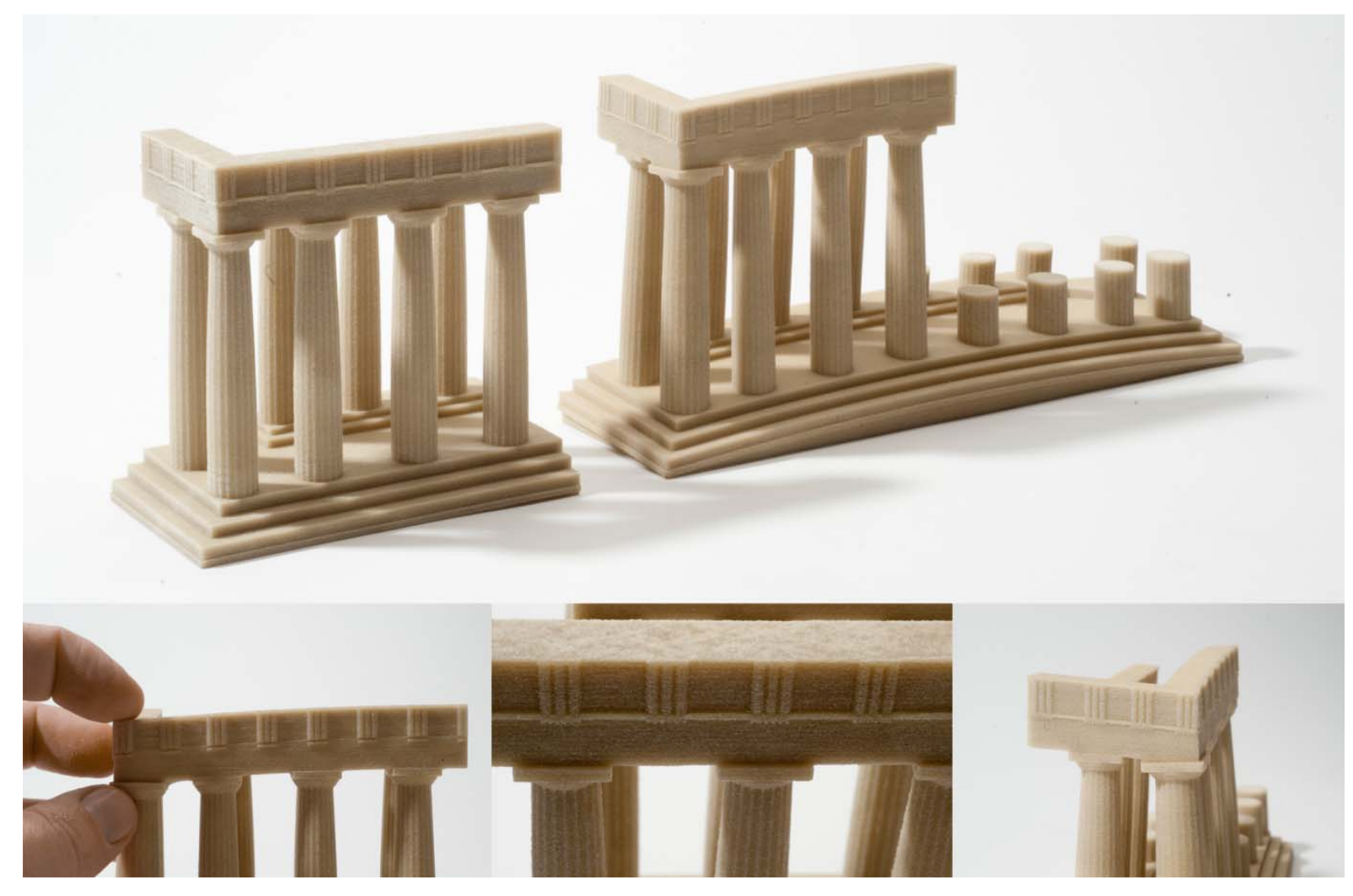

Figure 28. Final 1:150 prototyped models

(a) 'flat' and exaggerated, (b) curved Epistylia and Frieze, (c) detail view of Entablature, (D) corner detail 


\subsection{Analysis}

\section{Sources and objectives}

As the origin of the column study came from previous work, the initial problem description was clear and revolved around two distinct requirements: the necessity to produce visual recreations of the Parthenon through a particular medium, and the scale and scope of what was being recreated and reinterpreted. Two clear categories of historical source material were assessed: graphical and illustrative material, and accounts which sought to describe or 'unlock' the pattern of the Parthenon. Graphical material was the more accessible and stimulating of the two at an early stage as it gives a clear idea of the geometry involved. Accurate descriptions of layout, orientation, and the formal presentation conventions allow the Parthenon to be understood on an aesthetic and functional level of appreciation. Within the medium of digital parametric tools the accuracy and specificity of measurements were less helpful than the general layout and composition of elements. This was because parametric design seeks to set out variables and relate them rather than a simple modelling of explicitly stated geometry. The images of plans, sections, details and three dimensional images allow for an overview of the scale and complexity of the structure and provide a generalised, abstracted understanding of morphology and pattern.

The simplified Korres (fig 15) drawing illustrating the global refinements distilled from measurement and observation steps away from the tradition of exact replication of the observed and measured; it allows an audience to understand the structure on a deeper level and so was a critical piece of information in defining the problem statement. The project brief was heavily influenced by this Korres sketch and the goal of bringing the sketch to life in three dimensions was taken forward, whilst leaving the question of detail level and scope undefined. The involved process of creating variables and dependent geometry meant that the visual source material was influential and helpful in a general sense, but meant a deeper understanding of the relationships and probable intention and meaning of the Parthenon needed to be extracted from generalised textual accounts and theoretical analyses. Hence, an appreciation of the problems encountered during modelling influenced the search for sources which illuminate the plan and logic of the Parthenon set out, and thus the structure of the proposed model. This came about for two reasons; the notation of the medium (GenerativeComponents) being dense (a high degree of specificity and detail with a large 
library of methods) causing an iterative feedback loop, and the development of an understanding of how the specific refinements operate on both theoretical and practical levels. The problem encountered at this early stage was that of finding out how much was not known (within the project) and defining as much of this as possible without limiting the later developed model to a set of unsatisfactory possibilities.

\section{Problem development/ iterations}

The source material assisted in defining and understanding the project objectives and the direction of the modelling processes. The process of actually performing the tasks required within the GC modelling environment was more difficult and time consuming. Not only did the dense GC notation illuminate previously unexpected problems such as the required grammar of the recorded script and the simplification of geometric methods to a fixed library, these problems were fairly removed from the original goal and direction. These were technical challenges which had to be resolved in a way which did not compromise the intention of the overall project. The problem development phase following identification of technical issues was a time consuming process and involved trial and error and reflection within an iterative process.

The most pressing/ restrictive technical limitation was the calculability of unique and precise geometry as this was not observable until trial outputs of the column geometry were examined. This was first observed during an early attempt at modelling an integrated, inclined, and precisely trimmed set of columns as solid geometry. This also easily confounded the ability of GC and the typical computer processor to deal with a high degree of complexity. The learning process involved was more than a simple learning of which known methods were acceptable or not acceptable to the project; a compex process of finding at first unknown alternative methods was required. A major advantage to $\mathrm{GC}$ is the ability to intuitively construct new methods as subroutines based on the existing library of methods. Efficient models were required, but these were only possible after multiple iterations of inefficient (or even 'dead end') methods as illustrated in figs 20 to 23.

Another limitation which was primarily technical in nature was the difficulty in understanding how to limit the outputs to a format which took the virtual model into the physical world. This occurred as the final idealised model was prepared, exported and processed as a practical model. The purpose of the physical artefacts was to allow an 
audience to perceive the geometric refinements which are quite minor in dimension. The balance between scale, level of detail, and cost of production had to be balanced, as well as the overall objectives of the physical models. The minimum scope for illustrating the three most significant geometric refinements of Stylobase curve, column incline, and relative Entablature incline was set as pair of models of the short façade and Cella columns (fig 27). These models were assessed for volume and after several iterations and trial and error a scale of 1:150 was selected ${ }^{74}$. The 'flat' model was further trimmed to half width, and the 'exaggerated' model trimmed as a dog-leg section to allow full illustration of Stylobase curvature (fig 28).

Despite the large amount of published information relevant to defining the underlying principles of the parametric model it became apparent that this was indeed, at least in parts, a 'wicked' design problem. Firstly, there is an abstract problem definition which has no optimum solution; there is practically no 'best' solution, only better and worse ones which must be negotiated and reflected upon during the development of a series of iterative models. Secondly, the large scale variables at play (in the design process, not the parametric model itself) create conflicts and interact at a level which affects the overall project. These variables are the ability of the digital systems to compute complexity, the level of detail in the abstraction / idealisation of the model, the requirements of manufacture, the resources spent in constructing models and producing artefacts, and the aesthetic/ illustrative objectives of the study as a whole. These variables alongside the iterative problem solving methods outlined show that this project displays characteristics of an $\mathrm{N}$-dimensional problem with multiple assessment criteria. The parametric model can be seen as a sub-problem to the overall project and is also N-dimensional in itself. Lastly, the problem at the heart of the project is totally unique, and there are is no fixed set of methods to which can be applied. GC does contain a core set of fixed functions, but the ability to construct new methods from base principles as subroutines was essential in solving sub-problems.

The obstacles experienced in the case can be split into two categories; obstacles of tooling, which includes a sub-obstacle of learning, and obstacles to the production of useful, integrated outputs. These obstacles can be seen as barriers which must be negotiated in answering 'how' and 'why' a modeller builds a parametric model.

\footnotetext{
${ }^{74}$ On the $64 \mathrm{~mm}$ Stylobase curve (as in the Parthenon) at $1: 150 \mathrm{scale}=0.42 \mathrm{~mm}=2$ thin layers of fuse deposition - which are visible in 'flat' model
} 
The difficulties experienced can be summed up as the follows:

- Encoding the problem into the dense notation required by GC

- Training and learning involved in finding or creating methods

- Loosely applicable Heuristic methods can be involved

- Integrating the structure of the model so that useful outputs can be extracted which relates categorically to the source material and project objectives.

\subsection{Discussion}

In terms of comparing the observations within this case and the some theoretical models of design a few relevant lines of reasoning can be highlighted. Schon (1994) identifies a model of design which is a feedback loop of experience, learning, and practice. This can be observed within the Parthenon columns project as the process of iterative clarification of problem and solution spaces. The act of iterative development of both the project as a whole and the specific parametric model indicates that the practical test meets the theoretical predictions of Schon's reflective practice. In the case of the overall project this has taken the form of a several critical design moves which allow a certain output to be calculated and constructed as artefacts following a brief experimental stage which assisted in building an idea of the affordances provided by GC. The Parametric model development phase is noticeable in its use of multiple iterations and numerous edit/, repair/, replace operations. Each of these two models (the design model and the parametric model) required iterative development and reflection which influenced the other, and this process allows for the reconsideration of the role of artistry within the development of technical knowledge as in Schon (1995). The observable aspects of the case study match with the example of Schon's (1988) example, but the proposed definitions of different kinds of 'seeing' do not tightly match what is observed in the case. In Schon (ibid) 'recognize,' 'detect,' 'discover' and 'appreciate' denote variants of seeing which are indicative of the design moves taking place in this case.

An alternative model of design which can be seen as relating to design processes at a deeper level and incorporating a taxonomic classification of types of reflection and the consequences of design moves is Gero's Function-Behaviour-Structure (FBS) model (Gero 1998a, 1998b, 
2007). It draws on Asimov's (1962) Analysis-Synthesis-Evaluation model of design but seeks to break this down to observable design moves which reformulate the function $(\mathrm{F})$, expected behaviour $\left(\mathrm{B}_{\mathrm{E}}\right)$, actual behaviour $\left(\mathrm{B}_{\mathrm{S}}\right)$, structural $(\mathrm{S})$, or documentation $(\mathrm{D})$ characteristics as a result of reflection on design moves. Thus the Gero model of design incorporates design moves as they may be observed rather than more idealised or abstract ones. Examples from the Parthenon columns project follow:

- The requirement of solid geometry was identified at an early stage of column modelling (fig 22). The reformulation of the structure of the proposed model was reconfigured via an earlier structure. In Gero's model this would either equate to a $\mathbf{S} \rightarrow \mathbf{S}$ reformulation (structure to structure reformulation), or a $\mathbf{D} \rightarrow \mathbf{S}$ (reformulation of structure from documentation requirements)

- The scrapping of an inefficient column model (figs 22,24) can be seen as a reformulation of expected behaviour following an understanding of an actual behaviour after finding the behaviour was excessively computationally expensive. In Gero's model this would equate to a $\mathbf{B}_{\mathbf{S}} \rightarrow \mathbf{B}_{\mathbf{E}}$ reformulation (reformulation of the expected behaviours following analysis of the inefficient actual behaviour).

- Multiple iterations of the core relationships, which represents the majority of work in the case, can be seen as structural reconfigurations following actual or expected behaviours; $\mathbf{B}_{\mathbf{S}} \rightarrow \mathbf{S}$ or $\mathbf{B}_{\mathbf{E}} \rightarrow \mathbf{S}$ reformulations. These reformulations also include the majority of learning and exploration of methods during the project development.

- The selection and extraction of two illustrative models (the flat and exaggerated) from the final parametric model followed can be seen as the reformulation of documentation (D) (which in Gero's model follows from the Structure) via consideration of the Function; a $\mathrm{F} \rightarrow \mathrm{D}$ reformulation. (The documentation of the parametric model reformulated after re-considering the function of the entire project; particularly the scope).

The benefit of the Gero model of categorising reformulations is that one can observe not only that this happens on several levels, but that the types of interactions possible ( 8 types of reformulation are proposed in this $\operatorname{model}^{75}$ ) reflect the diverse design decision making strategies which are part of architectural design. Therefore the generalised obstructions

\footnotetext{
${ }^{75}$ See Gero (2007) for a full explanation
} 
observed in the case study can be related and understood within the Gero design model and the negotiation of obstacles can be understood as reformulations.

The obstacles experienced in this case study can be placed and explained within different design models. Despite any potential taxonomy of the position of obstacles, the core issues as they apply to parametric model making revolve around the ability to iteratively assess and rebuild parts of the model. This takes place via reformulation of the problem to which the model is addressing, including the degrees of freedom which the interface can provide, the acceptability of inaccuracies which can be caused by heuristic methods, and the overall function the model performs. While this is achieved in an iterative manner, there are some considerations which greatly affect how the task of modelling may occur. Appreciating the distinction between task based (tooling) and design output (integration) obstacles could allow faster development of the model and problem definition.

In terms of tooling, the degrees of freedom which a model accommodates is a primary constraining factor, and benefits from a clear and unambiguous problem definition. Undoubtedly, accommodating every possible variation and degree of freedom is not what is important in achieving a satisfactory result; rather normal design goals are the accommodation of useful and relevant variation. 'Unrestrained' and 'unlimited' parametric models would be problematic, and as such, loosely described modelling requirements would create extra work and require an extended iterative cycle.

Training and proficiency also have an impact on the obstacles encountered, and could conceivably restrict a design sequence. Finding and comparing modelling methods is in many ways a learning/ training process, particularly for unique or wicked problems. An example of this in this case study is the redevelopment of the individual column model which at first was horrendously inefficient; upon learning how the medium and model behaved a better, more efficient model was created from scratch. Finding the best solution for the task at hand can be difficult, but considering there are often no optimum solutions which are also practical means that considering heuristic methods can greatly lower the barriers to progressing the design solution. An example from this case study is the general use of slightly inaccurate heuristics in creating curves and splines in place of diminution methods.

In terms of obstacles to the integration of parametric models it is clear that considerations of the technical ability of computation are vital. The clearer these considerations are, the easier 
it is to select which aspect of the design problem can be solved through parametric models. An example from this case study is the restriction of the modelling scope to the three dimensional model to exclude Metope and pediment sculptures due to the non rational geometry freeform sculpture embodies. Another example is the requirement of the model, conceived before any modelling began, to produce a solid geometry which could be printed in $3 \mathrm{D}$ by machine. While this greatly constrained the model to the computational complexity of constructive solid geometry, the entire project would be difficult to present outside of a virtual or $2 \mathrm{D}$ environment otherwise.

Parametric design in this case can be seen as a trade off between flexibility (or degrees of freedom) and the ability to conceive, develop, compute, and present the final version of the parametric model. Generalising, limiting the problem space which a parametric model addresses would mean that the obstacles are easier to overcome.

\subsection{Conclusions}

While theoretical research in parametric design tends towards the general and positive, this practical case study highlights particular obstacles which may occur when designing with parametric tools. An early supposition that computational ability is nearly unlimited has not been observed, and so it cannot be the case that DPDTs remove the obstacle of design complexity. Although this is still a limiting factor to be considered when applying DPDT's, understanding other obstacles is nonetheless beneficial.

The case uses a design pattern which is relatively robust, and comprises a particular morphology which means that assessment of a particular structure is more straightforward than if the geometry of the problem addressed was loose or totally 'wicked' in nature. While there is no comparison within this case to such a loosely structured design problem to assess which is more difficult, it can be noted that the translation of design information into explicit parameters and hierarchical relationships from the wide body of source material clearly runs a logical course. This is mainly due to the rational and discrete nature of the Parthenon's anatomy, despite the minute-scale hyper refinements evident.

The more difficult obstacle comes from integrating the structure of the parametric model into something both relevant to the architectural problem and providing a useful output. In this case these two objectives seemed on the surface to be straightforward; bringing the Korres 
sketch to life as a three dimensional artefact through digital means. The effects of the design constraints on the iterative development of a series of parametric models was more complex, but essentially solvable once obstacles could be overcome. Building considerations as to which methods were both possible and efficient within the GenerativeComponents medium was essential in reflecting upon and rethinking the possible structure of parametric solutions.

Heuristics can be seen as influencing both types of obstacle. The translation of design information into an explicit parametric notation can take advantage of heuristics to progress the design and problem development. Heuristics are essentially patterns which can be used to test a proposed relationship as 'placeholders' and, if required, better heuristics can be developed at a later stage. The suitability of heuristic methods is a question of integration; in this case the loosely accurate methods did not compromise the output to a degree which causes the function of the model to become inadequate, and were negligible.

Despite limitations and extra work in using parametric methods to solve architectural problems, the various obstacles in exploring solutions in this manner pale in significance to the potential which certain tools and media can offer. Developing considerations of way in which a modeller can translate design constraints and parameters can help achieve some of this potential to provide more integrated solutions. 


\subsection{Thinking Through Digital Simulation Tasks in Architectural Education}

This chapter reports the activities of 80 second year architecture students at Victoria University Wellington for the duration of a single trimester. A central theme in this studio is the framing of day-lighting problems into a quantifiable investigation and then addressing these through the use of digital modelling and simulation tools. This study offers an insight to undergraduate architecture students' negotiation of digital design spaces and asks the question of how the knowledge of skill-based specialist tasks are extensible to core design studio and design process in general. The mass education within a University environment of such specialist skill based techniques allows for an insight to the negotiation of quantitative and qualitative design criteria, as well as allowing observation of obstacles and difficulties experienced in applying these skills. The issue of learning skill based tasks at university level is a pertinent topic of study as the critique of such techniques is implicit to the holistic education of Architects but the level of this critique can vary greatly. This question also highlights the challenges faced to improving the design education approaches to computational thinking and applications. It also questions the structure of ill-defined design problems as a trigger of difficulties; particularly the requirement for a designer to adapt one schema for another based on technical analysis. This paper finds that certain difficulties are experienced when technical workloads are increased which have the potential to obfuscate integrated design processes. Lastly, this paper concludes with remarks on how the process of lowering of technical barriers may affect reflection within design processes which incorporate technical assessments.

\subsection{Foreword}

Design simulations through the application of digital tools are becoming more well known and better utilised by training institutions which aspire to train larger groups of students in more complex assessment tools. The literature surrounding students negotiation and integration of digital tools within design assignments is somewhat limited; well known methods of simulation tend to reside within textbooks, or are cases from highly trained experts, and research publications which look at how the inexperienced use these tools are limited. The natural progression of digital tools to allow more complex forms to be 
represented and simulated has meant that students are more able to visualise unusual and exploratory designs and run environmental and other performative assessments. With this potential comes some drawbacks. Complexity is one aspect in which today's students can explore more readily, although the technical and cognitive load of advanced tools means that some other issues may become more difficult to address, such as translation of design ideas across media and more comp;ex discussions with collaborators (including design tutors as in the case presented). Design in general responds to a variety of parameters which constrain solutions, such as budget, site, and increasingly environmental performance criteria such as illumination requirements, but must negotiate the difficulties of particular media and communicating design moves. Difficulties experienced in this process can be constraining 'design parameters' in themselves. The act of Design as outlined in models of design such as Schon's (1995) Reflective Practice, Rowe's (1987) Design Thinking, and Gero's (1998a, 1998b) Function - Behaviour - Structure are conceived and carried out within an inherently iterative sequence of acts, and can be influenced and constrained by the tools used.

Digital tools can force new ways of working and can create new difficulties; the most noticeable is the technical nature and increased workload in building knowledge of these systems, which can. Digital tools, whether they are modelling, simulation, parametric, or programming-based can share several common issues. All these tools are interactive, and an interface must be negotiated in order to access them, which can sometimes be challenging to both use and understand. Further, these difficulties may exacerbate the separation between design thinking and execution, as the promise which more advanced tools offer in closing this gap can be dashed by interfaces which can get in the way. This section looks at how students interface with and negotiate difficulties or hurdles which are part and parcel of the more complex digital tools in the hope that wider lessons can be learnt in how these students are trained and also how digital tools can be integrated in a meaningful way in architectural design.

\subsection{Introduction}


Whilst daylight analyses have been previously applied to architectural applications mainly through heuristic methods ${ }^{76}$, newer tools have made this area more accessible to design studios and CAD based environmental science courses. This paper reports the activities of an undergraduate design studio which aims to have daylighting as a central theme; where lighting comfort and quality become an integral part of design problem solving. The traditional studio environment and professional culture can create a separation between disciplines; the design studio at the core and various specialised tasks, such as lighting or structural analysis as satellite events mainly to verify the feasibility of a design. Newer studio and teaching methods including descriptive analysis methods (Reinhart, 2006, 2009; Koutamanis, 2001) for environmental assessment have been applied in an attempt to overcome this obstacle and streamline students learning.

Undergraduate architecture degrees often contain core courses which require students to learn technical skills, develop understanding and to apply these to concurrent studio design papers. Human Environmental Science ${ }^{77}$ is one such paper taught at Victoria University Wellington NZ (VUW) School of Architecture at the second year level. The theoretical aspirations for such courses can risk being at odds with the actual level of achievement and application of design aid tools within the studio environment. The reasons for this can range from programme scheduling, cross-integration of assignments, tutorial size, tutor availability and the delivery of skills training. Whatever the issues or reasons, an effort can be made to improve the status quo and to convey the course content in a way which is more stimulating and rewarding to students.

At a time when class sizes are generally on the increase, higher levels of environmental analysis and technical outputs are becoming the norm. One concern highlighted through practice and previous research is the effectiveness of an iterative design methodology which utilises accurate descriptive environmental analysis for students to compare and refine solutions and the given design problem at the same time. Following from this, there is a concern as to the effectiveness of skill based training resources. This is because students may acquire the required technical skills to accurately assess environmental qualities but this in

\footnotetext{
${ }^{76}$ Dean Hawkes has provided an early history of mainly heuristic methods (1970) and also updated observations (2008); Hannah (1996) also outlines methods.

${ }^{77}$ See M Donn and J. Daish (1998) and Cuttle, C., Donn, M., and Baird, G. (1984) for the theoretical basis for the original 'Environmental Control Systems' course; now named 'Human environmental Science'.
} 
itself does not guarantee a grounded understanding or adequate motivation for more adventurous or 'creative' solution development.

\subsection{Background of Environmental Design Course}

Two decades ago the VUW architectural education programme was structured into courses that separated 'design' and 'science'. The latter papers focused on simple facts and design examples, examined independently from studio design ${ }^{78}$. The structured teaching of CAAD was also a separate activity relegated to communication courses. Design decision frameworks and other design methods described by authors (Koutamanis, 1993b; Gross, 1994) were intended to be developed in studio projects. The structure created a separation of technical competence by way of analytical skills and theoretical technical training and the 'core' design studio. Specific 'Integration' projects in studio required the students to synthesise this content.

In 1996, a radical restructuring of the architectural degree saw the Human Environment and Design courses amalgamated. This was intended to bring the integration processes, skills acquisition and analytical learning directly into the design Studio. A set of pragmatic lectures shared with Building Science and Interior Architecture classes formed the theoretical core of a poetic set of design projects in the Architecture studio. In these projects students were asked to tackle design tasks where the light, acoustic or thermal qualities were the principal focus. The projects were constructed around architectural challenges (for example "To develop a music chamber") using well-known architectural typologies found from an examination of precedent. The focus of the course was on the students' documentation through their design presentations not only of the poetry of their idea but of the calculated performance that met these goals.

The increased influence of digital applications in the programme was such that by 2003 the whole studio was digital; 3D model-based projects with digital output of drawings was the minimum requirement. For much of the decade 1998-2008, the course benefitted from an interest by the school in additional digital craft skills coaching in the second year of study.

\footnotetext{
${ }^{78}$ The 'old' system from 2 to 3 decades ago: separate courses in design and Environ mental Control Systems; each with own 'design exercises': whole buildings, windows (daylight), fittings (electric light), energy flows (heating/cooling) etc.
} 
The restructuring at VUW which took effect in 2010 separated the content back to two courses and provided the framework for the current system of integrated courses. This structure is described in Fig 29.

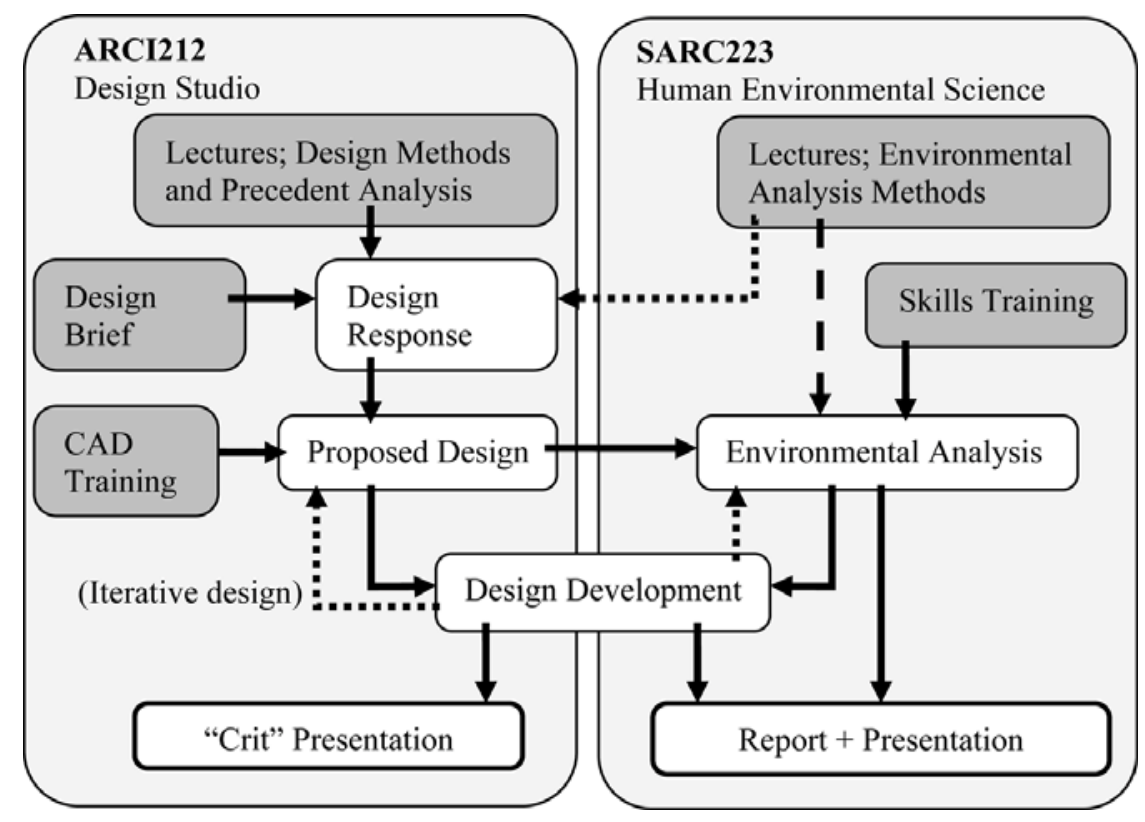

Figure 29. Diagram of current course structures

The current system could be characterised by an increasing focus on work processes and professional culture through pragmatic assignments as well as adaptation to updated methods of quantitative analysis. The goal is to take advantage of recent dramatic changes in the availability of CAAD and environmental analysis tools, as well as the ways in which studio culture operates. But the 'problem' of integration remains. The following hurdles of detailed digital environmental assessment have been identified as impacting on both the level of craft and integration of the two courses:

- Special training required and time consuming to set up

- An accurate analysis requires specific environmental information

- A developed 3D model is generally required

- There can be a level of uncertainty of results and accuracy

- 'Integration' advice from studio tutors / consultants can be limited

These issues are typical for a range of existing environmental assessment methods. Some of the inadequacies in Human Environment Design Tools previously highlighted (Paranandi, 
2001; Ramasubramanian et al, 1999; Novistski, 1990; Hanna, 2001) revolve partially around technological availability and problematic user interfaces and processes which have to some degree been overcome with the introduction of new software and hardware. The issues are still relevant to the new course structure; but two in particular which could be addressed within the courses were that of training and model complexity. Issues of training were addressed as this is the first step in overcoming the creative limits of the applied tool. Model complexity was addressed as the recently available programs can manage complexity to the point where students are not limited by their ability to design and create complex forms.

The approach is to develop the digital craft skills of the students in the same experiential manner that traditional craft processes and techniques are learned. The model is a continuation in the SARC223 paper of the digital craft learning exercises developed in its precursor courses ${ }^{79}$. The hurdles students are asked to overcome are reduced by using the best tool available. The training hurdle is the most critical and allows for proficiency rather than the 'pressing of buttons' to achieve an end result. Like any tool, understanding the obtainable results and the limits of these results requires building a working knowledge and implementation skills. Once the resulting accuracy of calculated data is unambiguous, students are likely to approach a level of working proficiency. This allows for information to be applied in a thoughtful way to influence design decisions in both quantitative and qualitative ways.

\subsection{Studio}

The following description of the studio course objectives is quoted from the course and project descriptions:

A studio based paper with a series of architectural design projects applying evaluative and critical processes in architectural design, building environmental design methods, and designing with a client brief. Principles of people-environment relationships; satisfaction and comfort; heat, light, sound....

\footnotetext{
${ }^{79}$ Familiarising students with the workable capacities of a digital medium is essential but takes time and practice (McCullough, 1996).
} 
Initially you will develop your design, and build a 3D CAD model of it, which is to be taken into the accompanying assignments in SARC 223, for the purposes of Daylighting and Acoustic analysis.

The second part of the assignment will be to continue to develop your design, and your CAD model, and to integrate into it the information you have learnt from Construction, Structures, and Human Environmental Science, and present a final scheme that is fully worked out.

Daylight and sunlight illumination is one aspect in particular where integration or bridging the gap can occur. The reasons for this are clear when one considers the effectiveness of quotes from great architects such as Le Corbusier, Scarpa or Khan in stimulating the poetic aspirations of students to apply themselves to the assigned design problem. One key precedent for this assignment presented and analysed in lectures was that of the Jubilee Line extension in London by various architects including Norman Foster; in particular the "Fosterino" entrance of the Canary Warf Underground Station. Through precedent analysis the students realise that there are pragmatic aspects to achieving such poetic ends; hence there may be some motivation to applying new techniques which reveal how these negotiations take place within a design process.

For this course the stance on digital tools was that the closer the language and medium of communicating the poetic and pragmatic aspirations of student designs the more likely that bridging of the gap can occur. This is because the formal visual abstractions and the analytical abstraction can now approach a near seamless transfer environment. The transfer of design intentions through iterative development of abstract models creates a situation where the barrier of translation is minimised and this can encourage 'bridging of the gap' to occur. Recent tools have been designed to allow for such translation operations.

\subsection{Methods for technique learning}

The approach to digital tools was applied in two specific ways not utilised in previous years; CAD teaching of Revit2010 in the studio paper and accurate lighting analysis in 3 dsMax. CAD learning was aimed at reinforcing the $3 \mathrm{D}$ modelling skills for both design development 
and streamlining environmental assessment through export processes. For the first time in the recent history of the course the requirement of all students to learn a CAD package for studio design was enforced. In recent years this was in effect only mandatory for the environmental assessments. Implementing mandatory 3D digital modelling in the studio had the effect of reducing the distance between the architectural design model and the analytical model.

Reinhart's (2009) verification of a method of accurate lighting analysis in 3dsMax allowed for the first time in this course a close family of programs in which studio design, visual representation and accurate lighting analysis could take place. A new approach was taken in the delivery of skills which addressed existing issues of demonstration and tutorial time limitations: on-demand tutorials via annotated YouTube videos. Previous experience highlighted the need for students to refer to 'on-screen' procedures rather than problematic and time consuming text based tutorials, whilst not relying on repeated demonstrations which would be necessary in large class sizes. The course structures limited the amount of time tutors could spend with students and so the emphasis on self-teaching and group teaching was increased relative to earlier years. Near the end of the studio design course specific questions were added to the regular University Training and Development Centre (UTDC) questionnaire to assess the perceived level of usefulness and integration of the skills taught through the online tutorials. Informal observations were also made through conversations with tutors and student representatives. These results and selected projects are described below.

\subsection{Results and student examples}

Informal observations regarding the students' studio culture can be summarised as follows. Generally, the enforced requirement for CAAD training within the studio in conjunction with new on-demand online tutorials created a situation where individuals intensively trained themselves to a competent level within a few days of the start of the course. As time went on, the students with higher levels of confidence began assisting those who were lagging behind. The result was accelerated student-led group learning beyond expectations; at a level which could easily be described as a 'first' in this course and the majority of students were able to 
set up accurate working lighting simulation models, albeit with simplified geometry, at an early stage. Responses to the survey questions ${ }^{80}$ are summarised in Figure 30.

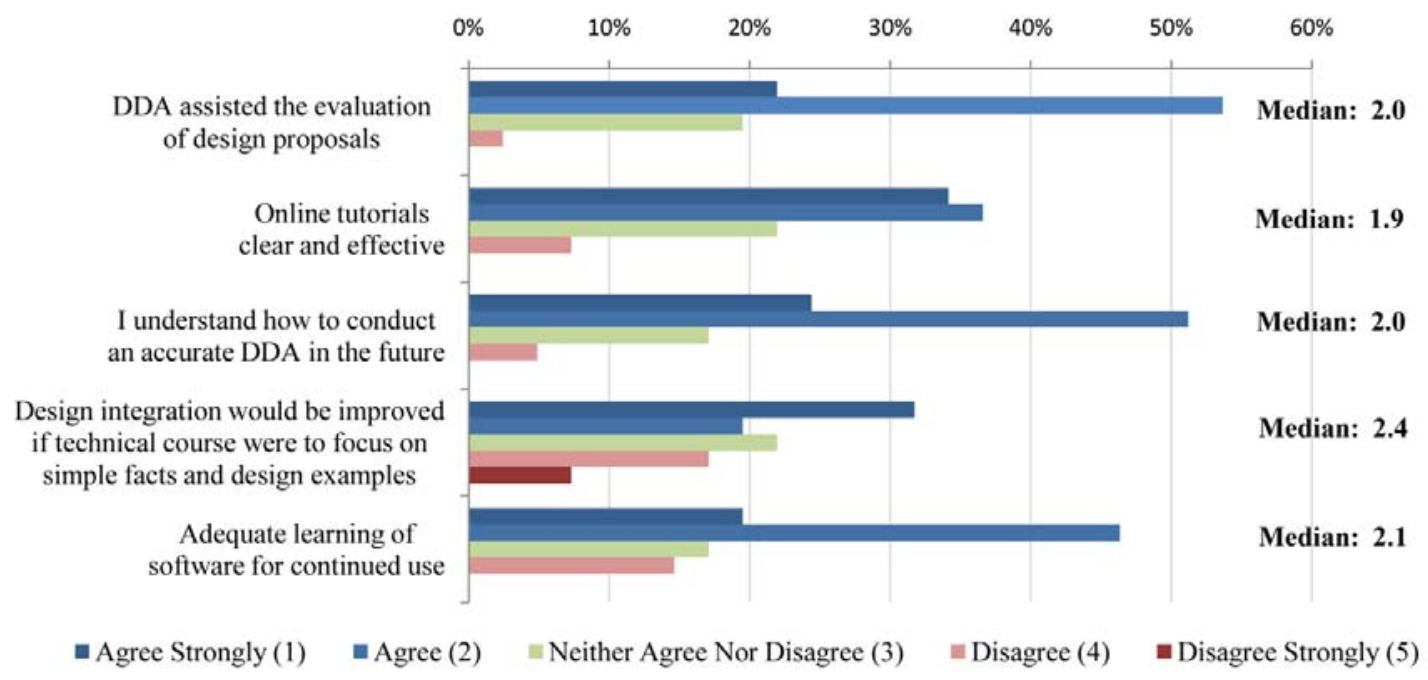

Figure 30. Survey responses (N=41) 'DDA' refers to Digital Daylight Analysis

These results reflected the students views that the analyses were useful (Q1), the online tutorials were helpful (Q2) and that assessment techniques would be used in the future $(\mathrm{Q} 3+\mathrm{Q} 5)$. There was some disagreement between respondents regarding Q4; the majority of students would prefer some aspects of the 'old' system of assessment; but a proportion think that the structure introduced for the first time this year is working.

Two student examples are highlighted; accurate lighting analyses were applied to iterative design processes. Figure 31 shows modification of a dynamic roof canopy following lighting assessment which indicated high light levels and contrast causing glare. Figure 32 shows modification of a series of shaped perforations in the ground plane for a subterranean station following lighting assessment which indicated low light levels and inappropriate quality of light distribution. Both students analysed the light levels across a range of dates and times

\footnotetext{
${ }^{80}$ The questions have been simplified for formatting reasons. The exact questions asked are as follows: (1) Lighting simulation analyses assisted the critical evaluation of design proposals in the core design studio projects.

(2) I am confident that, in future design projects in the school and later in practice, I understand how to conduct an accurate lighting analysis.

(3) I am confident that, in future design projects in the school and later in practice, I understand how to conduct an accurate lighting analysis.

(4) Design integration in ARCI 212 would be significantly improved if the technical course SARC 223 were to focus on simple facts and design examples, examined in essays and exams.

(5) I am confident that I have learned enough about the design analysis software like Ecotect that I can continue to practice on my own with other projects and develop and improve my skills on my own.
} 
and responded to verifiably accurate simulation results through modifications which were inline with response to brief and stated design intent.

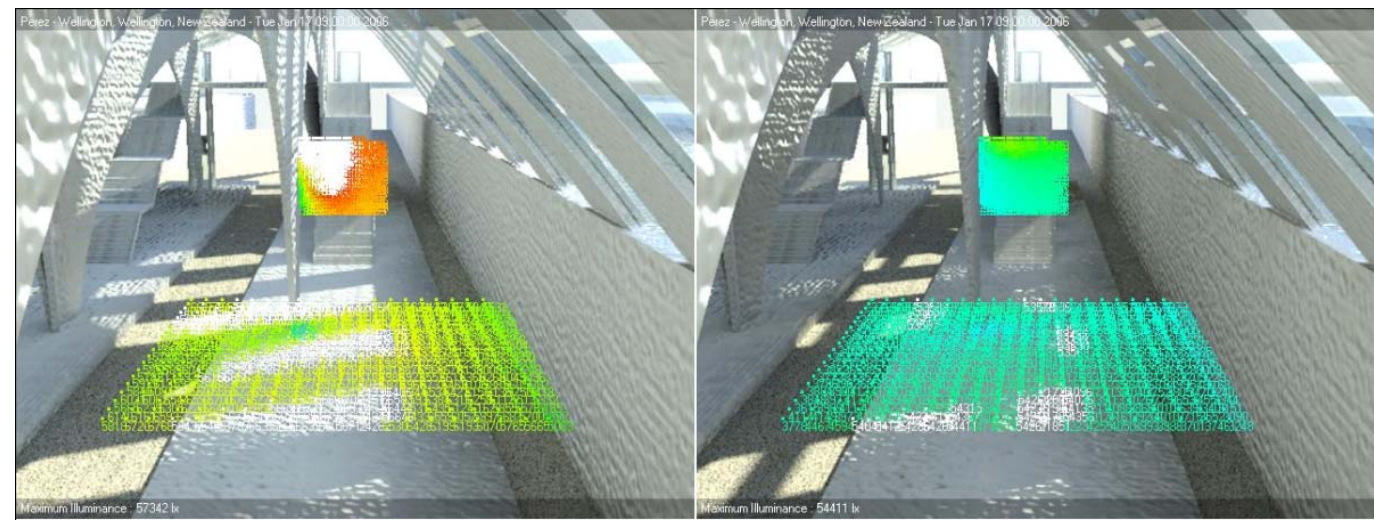

Fig 31; Monique Mackenzie covered transit station, showing illumination levels lighting analysis overlays; Iteration developed in response to contrast glare and roof fenestration pattern
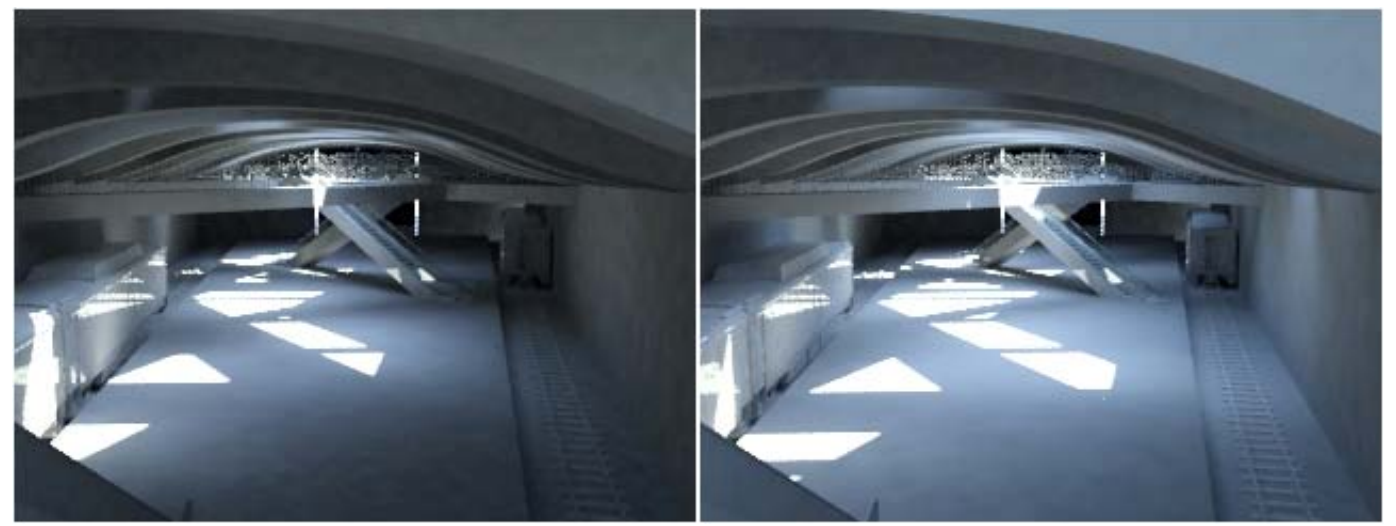

Figure 32. Alex Sawicka-Ritchie subterranean transit station. 'Common-sense' design iteration confirmed through analysis of light quantities and visual quality of distribution.

Comments and feedback from students primarily focused on the relative accessibility of the relevant digital tools. There was a perceived isolation during the first two weeks as student activities focused on training - learning programs. When the tools were applied to a concrete design scenario, skill acquisition sped up considerably, as fellow students collaborated to improve the proficiency level for the majority. At this stage a shift occurred from following the calculation tasks given to understanding why they are performed. One analogy given was that it is like a blind person following the directions of someone else, and then being able to see. 


\subsection{Analysis}

The difficulties experienced by the students at the beginning of the design process appear to primarily revolve around the technical workload and building of a working understanding of the digital tools in which simulations are performed. The modelling tools utilised allowed students to build large, complex models with a high degree of creative freedom. This is both positive in terms of widening the possible outcomes of a design process, and problematic as the possible configurations and variations which could be considered were vast. Students with a strong design concept could be typified as progressing through the learning stage quickly to achieve a three dimensional model which matches up with the hand drawn design plans and sketches common in architectural education. For these students, learning how to model three dimensionally was rapid due to a strong design direction; it could be said that these students already knew what they wanted before even starting to use the digital tools.

The reaction to simulation tools was markedly different than modelling ones in that the direction and understanding of what was to be expected was unknown at the beginning by students and to some degree the design studio tutors. Historical methods for analysing lighting performance, mainly through heuristics and hand calculations previously outlined in literature, did not resemble the process plans and sketches can produce in guiding the use of digital tools. The students had no intuition as to what to expect of a design move, and once simulations were performed many surprises were revealed; often the assessment illustrated that the design proposal would produce less natural light than expected. This was particularly observable for those students who had more poetic design concepts as these were perhaps less robust in the definition of what the design problem addressed. The process of simulating environmental characteristics was a more involved and strictly directed process than that of three dimensional modelling.

For the majority of students, learning how to integrate the information from simulation analyses to the design studio and their studio tutors was more difficult than digital modelling processes; this is suggested by the responses in fig 30, where learning how to use software appears easier than evaluating the actual proposals in a way tutors can understand. It is also suggested by feedback results and from the 'group' learning process some students tended to 
shy away from simulating multiple iterative models at an early stage, and the exercise changed from one of integration to one of proving after the fact that a design proposal met design requirements. Other students (as highlighted student examples illustrate in figs 31 and 32) embraced the surprises provided through simulation, and sought to redefine the design problem to balance the design constraints (including environmental) through a restructuring of the design proposal. This act of reflection matched acts within design models such as those proposed by Schon and Gero relatively closely. Translating design information between drawings, three dimensional models and simulation results created a situation where translating between media was, for the majority of students, a difficult process. Further, considering the role of studio tutors and classmates as collaborators, the process of translation also took on the extra obstacle of descriptive explanation of design iteration and reflection. Not only did the students have to modify the design iteratively from a weaker to a stronger solution, they were required to explain in a critique-led environment why and how these changes were made. Generally, students who had a rational and highly structured design concept, or who were working within well defined patterns, had fewer difficulties in translating between media and explaining these moves to others. Loosely structured, exploratory design proposals were more difficult to explain.

\subsection{Discussion and conclusions}

\section{Learning and using descriptive simulation methods}

The results presented in this paper show that the new system for integrating studio design and environmental assessment has had a positive effect in terms on student achievement and studio culture. In the past the inherent difficulties of environmental analysis restricted the ability of students to sincerely apply quantitative analyses to iterative design processes of the traditional studio. These difficulties have not been completely overcome but the negative effects can be moderated through new digital platforms and technique based training methods. There are two ways in which the students in this new system can benefit from breaking with traditions of environmental assessment integration with design studio.

Firstly, newer methods in delivering skill based training have improved the relationship of environmental science and architectural design studio through streamlining support and 
encouraging group-led knowledge acquisition. The specific methodology of on-demand video tutorials introduced in a sequential manner assisted in raising the abilities and confidence of the studio group as a whole. There are still barriers to integration which appropriate technical training cannot overcome. Conducting digital daylight analyses will confront students with a large amount of information; how they use this material in reporting to tutors is of concern and may be the weak link in the chain. Also, tutors of the studio design paper have a lower skills base in the analytical techniques than is asked of the students; this is because most of these tutors from industry rely on the 'standard' set of architectural skills to adopt their role as design mentors. They are unfamiliar with the environmental assessment methods. Previous courses within the School have successfully used senior students as skills coaches to fill this gap rather than extensive up-skilling of studio tutors, but the dual role of design and daylight expert is a more difficult one to fill.

Secondly, the switch to descriptive analysis tools has largely removed the simplification and trivialisation which can occur with previous heuristic methods. There is also an added benefit to using a single platform which can accommodate design exploration and accurate digital analysis, although this was not 'easy' or straightforward because of the multiple ways of designing and modelling across the student group. The user interfaces of the newer tools allow for quick up-take of required skill set; and allow for assessment and iteration cycles in short time frames.

\section{Generalising observed issues and difficulties}

In terms of notable difficulties and issues experienced by the students a few observations can be made. Firstly, the use of descriptive simulation methods rather than heuristic ones meant that there was an increased technical workload. On an individual level, this would be difficult to identify definitively but the nature of this case study looking at a large group of students highlighted that there were in fact technical difficulties beyond any general resistance to digital techniques; the group as a whole contained students who were skilled as well as unskilled in general digital media, and all experienced the 'hurdle' of technical capability in applying the specific analysis tool which had to be overcome. Further, it can be noted that there existed a range of approaches to design process, spanning the metaphorical, symbolic, rational, and tacit design drivers, and that all experienced some similar difficulties. 
These difficulties could be categorised as those of the technical and the integrative from the anecdotal evidence presented in this case. Whilst these categories may cross over, they appear as distinctly separate barriers due to the structure of students assignments and workloads. Generalising, students would be either 'working on the computers' performing simulation or 'at the desk' talking with design tutors and working on sketches or plans. This separation may be helpful in understanding how design aids or 'expert systems' can integrate within a wider design process where obstacles are overcome. The technical trait of learning how to use the descriptive, performative digital analysis tool preceded the involved sequence of linear acts required to run an accurate lighting simulation, as set out in the course and assignment structure. Students approached proficiency in digital craft slowly, and the process of achieving an initial set of simulation results was protracted, as suggested by the account of the learning process. Once some level of proficiency was attained, the students could build considerations as to the requirements of such an analysis, and factor this into the design process in a way which could streamline and enhance the iteration cycle. In this case the integrative difficulties were experienced following the technical, and made worse by some studio tutors advising students to ignore this technical requirement in the examination of design proposals.

The design process can be a difficult one. Observing the difficulties of digital tools, and advanced digital tools, is more difficult when observing students have less experience and a lower level of proficiency than other cases might. It is difficult to make conclusions as the nature of learning in this case study, as the issue is clouded by numerous factors including teaching style, curriculum structures and restructuring, the available and preferable tools available, the size of the student group, and the level of technical support. A more detailed study would be required to draw conclusive observations beyond the anecdotal. Further comparative studies of the same group of students completing similar tasks may further highlight what the effect of training has on the experienced difficulties.

The use of descriptive analysis systems is a more accurate way of assessing a proposed design when compared to simpler heuristic or 'hand-drawn' methods which are historically predominant. The ability to accurately assess the behaviour of a design is a technical step up; and allows for more complex and unusual design proposals to be assessed, and design schemas to be adjusted following accurate performative analyses which allow better insight into the performance of a proposed solution. In this way the design exploration space is 
expanded, but with a cost of higher learning requirements to overcome technical obstacles and difficulties. 


\subsection{Conclusions}

These conclusions address directly the original research question, highlighted literature, and hypothesis outlined in section one against the findings and evidence presented in section two and three.

Generally, we can observe from the cases that design development has occurred as an iterative, and at times loosely structured and defined process. The observations of practice within the two cases by and large match the design definitions and models outlined in theoretical and practical literature. Also, both the cases relied on both explicit and tacit processes but with contrasting order of application and iteration, again matching with design models and theoretical understandings of how unique and multivalent design problems are divided up and dealt with. Case one (section two) primarily subsisted an explicit process drawn out of a wider explorative study. The process of design iteration fitted closely within the scope of what is possible through explicitly parametric associative modelling, and required detailed, but straightforward iterative translations of source material into a digital medium. Further, this case utilised parametric design as an explicit programming process which sought to reduce the design variables and parameters to achieve an end result. Case two (section three) contrasted these characteristics (generalising the group of students) by beginning as a tacit process involving sketching, debate, and a wide variety of sources and ending up as an explicit model and simulation rig which differed significantly from iteration to iteration and from source to destination media. Further, the reduction of design variables within the design process was difficult to achieve, and the process of parametric design took place within the minds of students in a tacit way.

Broad design models from Schon, Rowe, and Alexander (and the like) correspond (unsurprisingly) with what has been observed in the two cases presented; essentially showing the nature of design iterations and exploration of tricky or wicked problems develops problem descriptions as much as the developing of solutions. In terms of the thesis research question, the broader design models could be characterised as hinting at some of the difficulties of design in general but lacking overarching principles.

Parametric design can be viewed as both an explicit process and a way of thinking; which responds to multiple criteria and constraints, and consist of the solving of $\mathrm{N}$-dimensional problems. Case one is explicitly parametric due to its medium (GC), as well as responding to multiple parameters such as scale, scope, budget, time, and aesthetic qualities. Case two 
displays the process of parametric design in two ways; the parametricised relationships encoded in both the standard three dimensional model making and simulation mechanisms as well as the process as a whole responding to the different design parameters of size and site restrictions, structural considerations, and design requirements such as lighting and acoustics. Both of these aspects can be seen as responding to information and understandings developed or uncovered (often through trial and error) which influences the structure of design solutions; and this can be seen in both presented cases.

\section{Specific points relating to hypothesis}

Hypothesis point 1: That there are observable obstacles inherent in the use of digital parametric design tools which impact of their use as architectural design aids.

Observable obstacles are evident in both cases, but they differ in character. These difficulties relate somewhat to what can be seen the theoretical literature, and more so to the practical literature. The distinction between tooling and integration obstacles proposed and distinguished in the cases are not particularly observable in literature amongst the numerous analyses and competing definitions and classifications. The suggested distinction, which on the whole is a logical step in nomenclature of difficulties, is not specifically helpful - apart from understanding which might be the more difficult facet of design process. These difficulties may impact the design process, but specific evidence presenting how they may affect the design process is not highlighted in the case studies.

Hypothesis point 2: The obstacles can be grouped into two generalised divisions; the obstacles of tool interface required to arrange models, and the obstacles faced when arranging a model to provide useful outputs to an iterative design process.

The distinction between the two obstacles 'tooling' and 'integration' can be seen in the two cases, but in actuality describe how technical information is integrated into the standard design process. This is obviously not something new, but the distinction is partially helpful in 
understanding where the 'extra work' may come from, and it can be observed in the two contrasting cases how this can occur to a limited degree:

Case 1 (section two) can be considered a relatively structured problem from the outset where the greatest difficulty, or obstacle is the integration with the objective of illustrating some of the Parthenon hyper-refinements. The technical translation, or tooling, was relatively straightforward due to the enormous body of research and the authors existing proficiency within the associative parametric medium. This is not to say that technical difficulties vanished in this case, indeed technical issues were time consuming at times problematic, but the core technical problem addressed was rational and well defined and so iterations and reflection had a strong assessment criteria and a clear direction. This may not have been the case if a lack of knowledge meant a greater level of training and learning was to take place alongside the design development.

Case 2 (section three) can be considered a relatively loosely structured problem (perhaps more accurately a group of problems) from the outset where the greatest obstacle was technical in nature. Students in the study primarily were for the majority more comfortable working on a tacit, loosely structured series of iterations which took priority over technical information and environmental simulation. Poetic influences dominated the development stage and the role of tooling technical assessment solutions followed mostly complete conceptual designs. A complicating issue was that the students were learning for the first time how to operate specific environmental software, and as such were focussed on the interface layer of a more complicated set of mechanisms which lay underneath. If this were not the case, and proficiency was at a high level before design instigation, then there would perhaps have still been a preferred working process which existed on a tacit, poetic level due to the tendencies of relatively 'green' designers.

\section{Hypothesis point 3: Education/training plays a role in conjunction with these two obstacles.}

Learning does have a role to play in the iterative design process on two levels. Proficiency of a designer before a specific design clearly has an effect in terms of experience, technical capability, and the considerations developed through previous practice. Case one is an example where the designer (author) has a mid to high level of proficiency, where case two involves designers who are not proficient in environmental simulation from the outset. 
Learning has a role to play in the iterative reflection of a multifaceted, multidimensional design problem; as a designer learns through diverse means what can or possibly could be a satisfactory solution to the design problem, and the development of the problem itself. The existence of some relationship between these can be gleaned from the case studies, although this is anecdotal evidence at best, and conclusions cannot be drawn as to the nature of learning in the design process from the evidence presented here, or if this can be seen as an obstacle in the same way as the proposed tooling or integration are.

The distinction of difficulties could be used in highlighting which areas are more difficult to different types of problems in the planning of educational programmes. By understanding a process better a designer who is learning may be more likely to build considerations as to process which can be accommodated easily, and what may be a more tenuous or vague process. Anticipating this process of how considerations are constructed stage by stage in the designers mind could therefore assist in the process of training.

Hypothesis point 4: The core obstacle to integrating DPDT's is the ability of the modeller to adapt one schema as the design is explored and requirements change, and thus, the effects of tool interface and design process interface can be accounted for and anticipated to allow smoother and better integration within the design process.

Case one illustrates that some difficulties such as those of a technical nature can be anticipated from the outset, but integrative difficulties may not be clear until several design iterations occur. Case two illustrates how integrative and technical issues can be anticipated from the outset but technical difficulties are not truly appreciated until after both the initial training hurdle and the detailed application of technical assessments occurs. The cases may illustrate that there can be an understanding of which type of obstacle is occurring at a certain stage, but there is little evidence as to how understanding the difficulties can be utilised to improve the control of the design process. If a designer could anticipate which type of problems might be the greater before a design process commenced then there could be some consideration as to which problems, or which parts of problems, could be solved effectively. 


\section{Hypothesis point 5: The effects of the core obstacle can be minimised by following strong}

patterns which limits the unknown quantities. Less well defined problems and model patterns encounter greater obstructions.

Strong patterns seem to help restrict or remove obstacles to a degree. It can be observed in a limited manner by the difference in the cases. As suggested by Woodbury, patterns appear to hold some of the answers to dealing with issues surrounding problem descriptions: insomuch as there is some clear guidance to the designer who seeks to stay within the pattern. For case one the pattern is rigid and the process explicit and so an end state is clear once arrived at. For case two the pattern is loosely defined (if at all) and the process is tacit. There are obvious limitations to this analysis; that the comparison is not like to like and this could be more robustly addressed by further comparison of the existing cases with those which comprise rigid patterns in a more tacit process, and loose patterns within a more explicit process. The supposition that loosely defined processes require greater effort in overcoming difficulties cannot be proved or disproved, but anecdotal evidence from case one suggests that this may be the valid situation.

Thus, to return to the overall research question: "What are the characteristics of some of the observed issues and obstacles revealed in the practical application of digital parametric design tools in architecture?" the following observations can be made.

The case studies presented here have illustrated that these difficulties are inherent to explicit parametric modelling through programming (case one) and digital parametric modelling in general as an extension of thought processes within design (case two); it has been possible to draw some lessons of the nature these difficulties to propose naming some of them as those of tooling, and some as those of integration.

Another lesson which can be drawn from the cases is that the nature of the difficulties can be difficult to pin down, in part due to the unique settings in which design takes place. This research therefore suggests that pursuing this participant observer approach to case study research of explicitly parametric design in a group environment will show more about how these difficulties might be experienced. 


\section{// Bibliography}

All websites listed accessed 01/01/2012

AACA (2006) Australian Architecture Program Accreditation and Recognition Procedure http://www.architecture.com.au/i-cms_file?page=649/Acced_bk_06_web_version.pdf

Achten, H., Koszewski, K., Martens, B. (2011a). What happened after the "Hype" on Virtual Design Studios?: Some Considerations for a Roundtable Discussion. Respecting Fragile Places;

eCAADe2011 Conference Proceedings, University of Ljubljana, Faculty of Architecture (Slovenia), pp.23-32.

Achten, H. (2011b). Degrees of Interaction: Towards a Classification. Respecting Fragile Places; eCAADe2011 Conference Proceedings, University of Ljubljana, Faculty of Architecture (Slovenia), pp.565-572.

AIA (2009). Interoperability Position Statement. AIA Board of Directors Dec 2009. http://www.aia.org/aiaucmp/groups/aia/documents/pdf/aiab082297.pdf

Aish, R., Shea, K., and Gourtovaia, M. (2003). Towards Integrated Performance-Based Generative Design Tools. Digital Design: The Quest for New Paradigms; eCAADe 2003 Conference Proceedings, Graz (Austria). pp. 553-560.

Aish, R. (2005) From Intuition to Precision. Digital Design: The Quest for New Paradigms; eCAADe 2005 Conference Proceedings, Lisbon (Portugal), pp. 10-14.

Aish, R. (2006). Bentley's GenerativeComponents. SmartGeometry Conference. London: Bentley Systems Institution.

Aish, Robert (2009) Tools of Expression: Notation and Interaction for Design Computation. reForm( ) - Building a Better Tomorrow; ACADIA 2009 Conference Proceedings, Chicago (Illinois), pp. 30-31.

Alexander, C. (1964). Notes on the Synthesis of Form. Cambridge, Massachusetts: Harvard University Press.

Alexander, C. (1977). A Pattern Language: Towns, Buildings, Construction. New York: Oxford University Press.

Anay, H. (2010). Computational aspects of a design process: Mario Botta's single-family house in Breganzona. CAADRIA 2010 Conference Proceedings, Hong Kong, pp. 49-58.

Anders, P. (2003). Four Degrees Of Freedom. Connecting $>>$ Crossroads of Digital Discourse; ACADIA 2003 Conference Proceedings, Indianapolis (Indiana), p. 17.

Angelopoulos, A. (2003). Metron ariston: ho agnōstos megalithikos pēchys kai ta aporrēta mystika tès Hellēnikēs architektonikēs. Athens: Ekdoseis "Aeropos".

Asimow, M. (alt. spell. ‘Asimov’) (1962). Introduction to Design.New Jersey: Prentice-Hall.

Balanos, N. (1940). H $\alpha v \alpha \sigma \tau \dot{\lambda} \omega \sigma l \varsigma \tau \omega v \mu v \eta \mu \varepsilon i \omega v ~ \tau \eta \varsigma ~ A \kappa \rho o \pi o ́ \lambda \varepsilon \omega \varsigma ~ I-I I$, Athens. 
Bhatt, A., Kishore, A.V.V. (2008). Studying corpus changes in CumInCAD. eCAADe 2008

Conference Proceedings, Antwerpen (Belgium), pp. 855-860.

Bhatt A., Martens, B. (2006). ON-TO-CAAD: Investigating the Knowledge Contained within the Corpus of CAAD Research. CAADRIA 2006 Conference Proceedings, Kumamoto (Japan), pp. 413424.

Bhatt, A., Martens, B. (2009). The topics of CAAD: An evolutionary perspective - a research for representing the space of $C A A D$. Tidafi and Dorta (Eds.) Joining Languages, Cultures and Visions: CAADFutures 2009 Conference Proceedings, pp. 626- 641.

Burry, M. (2003). Between intuition and process: Parametric design and rapid prototyping. in Kolarevic B. (Ed.) Architecture in the digital age - design and manufacturing. Abingdon: Taylor and rancis, pp. 147-162.

Burry, M. (2005) Digitally Sponsored Convergence of Design Education, Research and Practice. CAADFutures 2005 Conference Proceedings, Vienna (Austria) pp. 3-22.

Burry, J., Burry, M. (2006). Sharing hidden power - Communicating latency in digital models. Communicating Space(s): eCAADe Conference Proceedings, Volos (Greece), pp. 786-793.

Burry, J., Burry, M. (2008). The Bonds of Spatial Freedom. eCAADe 2008 Conference Proceedings , Antwerpen (Belgium), pp. 301-308.

Burry, J., Burry, M. (2010). The New Mathematics of Architecture. London: Thames \& Hudson.

Burry, J., Burry, M. (2011). Scripting Cultures: Architectural Design and Programming. New York: Wiley.

Carrey, J. (originals 1674) drawings in Bowie, T.R., Thimme, D.(1971), The Carrey Drawings of the Parthenon Sculptures. Indiana: Indiana University Press.

Chang, W-T., Chang, T-W. (2006). Folding Space With Time-Based Operations, CAADRIA 2006 Conference Proceedings, Kumamoto (Japan), pp. 569-571.

Cheng, R. (2006). Report on Integrated Practice, Suggestions for an integrative education. Washington, DC: American Institute of Architects.

Christenson, M. (2011). Parametric Variation Revealing Architectural Untranslatability.

Parametricism; ACADIA 2011 Conference Proceedings, pp. 267-273.

Collins Dictionary, $11^{\text {th }}$ ed. (2011). Glasgow: HarperCollins.

Coulton, J. J. (1977). (fig. 44.) in Ancient Greek Architects at Work: Problems of Structure and Design. Ithaca: Cornell University Press:

Cuttle, C., Donn, M., Baird, G. (1984). Environmental Science Design Exercises. ANZAScA 1984 Conference Proceedings, Sydney: Australia and New Zealand Architectural Science Association.

Davis, D., Burry, J., Mark, B. (2011a). The flexibility of logic programming : Parametrically regenerating the Sagrada Familia. CAADRIA 2011 Conference Proceedings, Newcastle (Australia), pp. 29-38.

Davis, D., Burry, J., Mark, B. (2011b). Untangling Parametric Schemata: Enhancing Collaboration Through Modular Programming. CAADFutures 2011 Conference Proceedings, Liege (Belgium), pp. 55-68. 
Debevec, P., Einarsson, P., Hawkins, T. (2004). Photometric Stereo for Archeological Inscriptions. SIGGRAPH 2004 Conference Proceedings, Los Angeles (USA).

Debevec, P. (2005). Making "The Parthenon". Proceedings of the 6th International Symposium on Virtual Reality, Archaeology, and Cultural Heritage, Pisa, (Italy).

Deleuze, G. (c. 1988). Le pli : Leibniz et le Baroque, Paris : Editions de Minuit. Translated by Conley, T. (2006). The fold : Leibniz and the Baroque. London, New York: Continuum.

Dinsmoor, W. B. (1902). The Architecture of Ancient Greece. (Third revised edition 1950), London: B.T. Batsford.

Donn, M., Daish, J. (1998). The Sensory into Architecture - Ars Sine Scientia Nihil Est. ANZAScA 1998 Conference Proceedings, Wellington: Australia and New Zealand Architectural Science Association.

Euclid (300 BC), Elementa (fragment) (888). Byzantium. The Bodleian Library Collection, University of Oxford.

Fernando, R., Drogemuller, R., Salim, F. D., Burry, J. (2010). Patterns, heuristics for architectural design support: making use of evolutionary modelling in design. CAADRIA 2010 Conference Proceedings, Hong Kong SAR, pp. 283-292.

Feng, H. (2010). Quantum architecture: an indeterministic and interactive computational design system. CAADRIA 2010 Conference Proceedings, Hong Kong SAR, pp. 619-628.

Fischer, T., Burry, M., Frazer, J. (2003). How to Plant a Subway System. Digital Design - Research and Practice; CAADRIA 2010 Conference Proceedings, Tainan (Taiwan), pp. 403-412.

Fischer, T. (2008). Designing (tools (for designing (tools for ...)))). PhD Thesis, RMIT University, Melbourne.

Fraser, M., Donn, M. (2011). Thinking through digital simulation tasks in architectural education. CAADRIA 2011 Conference Proceedings, Newcastle, (Australia), pp. 599-608.

Foster and Partners, et al. (c. 2004). Swiss Re Bulding. Retreived from:

http://www.fosterandpartners.com/projects/1004/default.aspx

http://www.arup.com/Projects/30_St_Mary_Axe.aspx

Foster and Partners, et al. (c. 2004). Greater London Authority Headquarters. Retreived from: http://www.fosterandpartners.com/Projects/1027/Default.aspx

Frazer, J.H. (1995). An Evolutionary Architecture. London: Architectural Association.

Friedman, D. (2006). Report on Integrated Practice, Architectural Education and Practice on the Verge. Washington, DC: American Institute of Architects.

Gero, J. S. (1990). Design prototypes: a knowledge representation schema for design. AI Magazine, 11(4), pp. 26-36.

Gero, J.S. (1998a). Conceptual designing as a sequence of situated acts. in Smith, I. (Ed.), Artificial Intelligence in Structural Engineering. Berlin: Springer, pp. 165-177.

Gero, J.S. (1998b). Towards a model of designing which includes its situatedness. in Grabowski, H., Rude, S., Green, G. (Eds.), Universal Design Theory, Aachen: Shaker Verlag, pp. 47-56. 
Gero, J.S., Kannengiesser, U. (2007). A Function-Behavior-Structure Ontology of Processes.

Artificial Intelligence for Engineering Design, Analysis and Manufacturing : AI EDAM, 21 (4), pp. 379-391.

Glymph, J., Shelden, D., Ceccato, C., Mussel, J., Schober, H. (2002). A Parametric Strategy for Freeform Glass Structures Using Quadrilateral Planar Facets. Thresholds between Physical and Virtual; ACADIA 2002 Conference Proceedings, Pomona (California), pp. 303-321.

Goldschmidt, G. (1990). Linkography: Assessing Design Productivity. Cyberbetics and System 1990 Conference Proceedings, Singapore: World Scientific, pp. 291-298.

Gross, M. (1994). Roles for Computing in Schools of Architecture and Planning. Journal of Architectural Education, 48 (1), pp. 56-64.

Hanna, R. (1996). A Computer-based Approach for Teaching Daylighting at the Early Design Stage. Education for Practice; eCAADe 1996 Conference Proceedings, Lund (Sweden), pp. 181-190.

Hanna, R., Barber, T. (2001). An Inquiry into Computers in Design: Attitudes Before-Attitudes After. Design Studies, 22 (3), pp. 255-281.

Hawkes, D. (1970). A History of Models of the Environment in Buildings. Working Paper 34; The Centre for Land Use and Built Form Studies in the University of Cambridge.

Hawkes, D. (1998). The Environmental Tradition: Studies in the Architecture of Environment. London: E \& FN Spon.

Hernandez, C.R.B. (2006). Thinking parametric design: Introducing Parametric Gaudi. Design Studies, 27 (3), pp. 309-324.

Hesselgren, L., Charitou, R., Dritsas, S. (2007). The Bishopsgate Tower Case Study. International Journal of Architectural Computing, 5 (1), pp. 62-81.

Hudson, R. (2011). Strategies for parametric design in architecture. PhD thesis, University of Bath.

Hurwit, J.M. (2000). The Athenian Acropolis: History, Mythology, and Archeology from the Neolithic Era to the Present. Cambridge University Press.

Hurwit, J.M. (2005). The Parthenon and the Tample of Zeus at Olympia. In Barringer, J. M., Hurwit, J.M., Pollitt, J.J. (Eds.), Periklean Athens and Its Legacy: Problems and Perspectives. University of Texas Press.

Jabi, W. (2007). The Challenges of Design and Computation. Retreived from: http://wjabi.net/Challenges.pdf.

Kan, J.W.T., Gero, J.S. (2005). Can Entropy Indicate the Richness of Idea Generation in Team Designing? CAADRIA 2005 Conference Proceedings, New Delhi (India), pp. 451-457.

Karle, D., Kelly, B.M. (2011). Parametric Thinking. Parametricism; ACADIA 2011 Conference Proceedings, Calgary/Banff (Canada), pp. 109-113.

Katz, N.C. (2010). Algorithmic Modeling; Parametric Thinking: Computational Solutions to Design Problems. CAAD - Cities - Sustainability; ASCAAD 2010 Conference Proceedings, Fez (Morocco), pp. 19-36.

Kilian, A. (2006a). Design Exploration With Circular Dependencies: A chair design experiment. CAADRIA 2006 Conference Proceedings, Kumamoto (Japan), pp. 217-226. 
Kilian, A. (2006b). Design Exploration through Bidirectional Modeling of Constraints. PhD Thesis, Massachusetts Institute of Technology.

Kolarevic, B. (2003). [Chapters 1-4] Architecture in the digital age-design and manufacturing. Abingdon: Taylor and Francis.

Kolarevic, B. (2008). The (Risky) Craft of Digital Making. In Kolarevic, Klinger (Eds.) Manufacturing Material Effects: Rethinking Design and Making in Architecture. New York: Routledge, pp. 119-128.

Korres, M. (1992). Construction of ancient columns. (English title) Oikodomikē tōn archaiōn kionōn. (Greek title) Athens: (publisher unknown; possibly Ephorate of the Acropolis Antiquities)

Korres, M. (1993). Refinements of Refinements., at the second Williams Symposium, also in: Appearance and Essence. Refinements of Classical Architecture: Curvature. Haselberger, L. (Ed.) (1993). The University Museum, University of Pennsylvania.

Korres, M. (2000). The stones of the Parthenon. Los Angeles: Getty Trust Publications: J. Paul Getty Museum.

Koutamanis, A. (1993a). On the Correlation of Design and Computational Techniques in Architectural Education. eCAADe 1993 Conference Proceedings, Eindhoven (The Netherlands), pp. $25-27$.

Koutamanis, A., Mitossi, V. (1993b). Computer vision in architectural design. Design Studies, 14(1)

Koutamanis, A. (2001). Analysis and the descriptive approach. AVOCAAD 2001 Conference Proceedings, Brussels (Belgium), pp. 72-81.

Kowalski, R. (1979). Algorithm=Logic + Control. Communications of the Association for Computing Machinery (ACM) 22 (7): pp.424-436.

Li, M., Vita'nyi, P.M.B. (1997). An Introduction to Kolmogorov Complexity and Its Applications. 2nd ed. New York: Springer-Verlag.

Lawson, B. R. (1994). Design in Mind. Oxford: Butterworth Architecture.

Mahdavi, A., Gurtekin, B. (2002). Shapes, Numbers, Perception: Aspects and Dimensions of the Design-Performance Space. Design and Decision Support Systems Conference Proceedings; Part one: Architecture. Ellecom (Netherlands).

Maleki, M.M., Woodbury, R.F. (2010). Programming in the model: combining task and tool in computer-aided design. CAADRIA 2010 Conference Proceedings. Hong Kong, pp. 117-125

Markowsky, G. (1992). Misconceptions about the Golden Ratio. The College Mathematics Journal 23 (1), pp. 2-19.

Martens, B. (2004). Cumincad Hacks. SIGraDi 2004 Conference Proceedings, Porte Alegre (Brasil), pp. $487-492$.

Martens, B., Koutamanis, A., Brown, A. (2007). Predicting the Future from Past Experience. Predicting the Future; eCAADe 2007 Conference Proceedings, Frankfurt (Germany), pp. 523-531.

McCullough, M. (1998). Abstracting craft: the practiced digital hand. Cambridge, Mass.: MIT Press. 
Mihova, J., Fraser, M. (2008). Making Sense of the Multilayered Past. An Interactive Visual Interpretation of the Athenian Acropolis and the Parthenon. Presentation at the 6th International Conference on History: From Ancient to Modern 2008 Conference Proceedings, Athens (Greece): Athens Institute for Education and Research

Mihova, J., Fraser, M. (2011). Scale(s) of Cultural (re) Productions Liberties and limitations in applications of contemporary technology. DMACH 2011 Conference Proceedings: In Digital Media and its Applications in Cultural Heritage, Eds J. Al-Qawasmi, Y. Alshawabkeh, F. Remondino; Amman: CSAAR Press, p. 185.

Mitchell, W. (1990). The Logic of Architecture : Design, Computation, and Cognition. Cambridge, Mass.: MIT Press.

Mueller, I. (1981). Philosophy of Mathematics and Deductive Structure in Euclid's Elements. Cambridge, Mass.: MIT Press.

Mueller, V., Johnson, S. (2000). Binary Oppositions: Are Computers Yet Aids for Design? ACADIA Quarterly, vol. 19, no. 3, pp. 4-6

Mueller, V., Talbott, K. (2003) Architectural Design Methods with Commercial Computer Aided Design Systems. eCAADe 2003 Conference Proceedings, Graz (Austria), pp. 279-286

Mueller, V. (2009a). Conceptual Design Tools: Establishing a Framework for Specification of Concept Design Tools. Digitizing Architecture Formalization and Content; ASCAAD 2009 Conference Proceedings, Manama (Kingdom of Bahrain), pp. 103-120.

Mueller, V. (2009b). Ontology for Computational Design: Computational Methods versus Cultural Processes. Computation: The New Realm of Architectural Design; eCAADe 2009 Conference Proceedings, Istanbul (Turkey), pp. 441-448.

Mueller, V., Iordanova, I. (2011). Rethinking concept design tools: High-level requirements for concept design tools. CAADRIA 2011 Conference Proceedings, Newcastle (Australia), pp. 409-418.

OED. (2002). Oxford English Dictionary. Oxford: Oxford University Press.

Oxman, R. (2010). The New Structuralism: Conceptual Mapping of Emerging Key Concepts in Theory and Praxis. International Journal of Architectural Computing 8(4) p. 419.

Pearl, J. (1983). Heuristics: Intelligent Search Strategies for Computer Problem Solving. New York: Addison-Wesley.

Penrose, F. (1888). An Investigation of the Principles of Athenian Architecture; The Results of a Survey conducted Chiefly with Reference to the Optical Refinements Exhibited in the Construction of the Ancient Buildings at Athens. Second edition. London: Macmillan, (first edition 1851).

Penrose, R. (2005). The Road to Reality: a complete guide to the laws of the universe. Berlin: Jonathan Cape.

Psycharis, I.N., Papastamatiou, D.Y., Alexandris A.P. (2000). Parametric investigation of the stability of classical columns under harmonic and earthquake excitations. Earthquake Engineering and Structural Dynamics 29: pp. 1093-1109.

Reinhart, C. F., Fitz, A. (2006). Findings from a survey on the current use of daylight simulations in building design. Energy and Buildings, 38(7), pp. 824-835.

Reinhart, C., Breton, P-F. (2009). Experimental Validation of Autodesk ${ }^{\circledR}$ 3Ds Max ${ }^{\circledR}$ Design 2009 and 
Daysim 3.0. Autodesk. Retrieved from:

http://www.autodesk.com/us/3dsmaxdesign/B3241.MentalRayValidation_v3.pdf

RIBA (2010). Validation Criteria. Retrieved from:

http://www.architecture.com/Files/RIBAProfessionalServices/Education/Validation/RIBAValidation CriteriafromSeptember2011Parts1,23.pdf

Rittel, H.W.J., Webber, M.M. (1973). Dilemmas in a General Theory of Planning. Policy Sciences, Vol. 4. Amsterdam: Elsevier Scientific Publishing Company, pp.155-169.

Rowe, P.G. (1987). Design Thinking. Cambridge, Mass.: MIT Press.

Sakamoto, T., Ferre, A., Kubo, M. (eds.). (2008). From Control to Design: Parametric/Algorithmic Architecture. Barcelona: Actar.

Schach, S. R. (2008). Object-Oriented Software Engineering. New York: McGraw-Hill.

Schoch, M., Praditsmanont, A., Prakasvudhisarn, C. (2010). Shaping building volumes through life cycle costs: a constraint programming approach for building volume optimisation. CAADRIA 2010 Conference Proceedings, Hong Kong, pp. 185-194.

Schon, D.A. (1983). The Reflective Practitioner: How professionals think in action. London: Temple Smith.

Schön, D.A. (1995). The reflective practitioner: how professionals think in action, (revised edition) Aldershot: Arena.

Schumacher, P. (2008). Smart work - Patrik Schumacher on the growing importance of parametrics. Riba Journal, September 2008.

Schumacher, P. (2010). The Autopoiesis of Architecture Vol 1: A New Framework for Architecture. New York: Wiley.

Shea, K., Aish, R., Gourtovaia, M. (2003). Towards integrated performance-driven generative design tools. Automation in Construction 14 (5) pp. 253-264. (Also in) Digital Design: eCAADe 2003 Conference Proceedings, Graz (Austria), pp. 553-560.

Shelden, D. (2002). Digital Surface Representation and the Constructability of Gehry's Architecture. $\mathrm{PhD}$ Thesis, MIT, Cambridge, Mass.

Smith, A.H. (1908). A Guide to the Sculptures of the Parthenon in the British Museum. British Museum. Dept. of Greek and Roman Antiquities. London: W. Clowes.

Stuart, J., Revett, N. (1762). The Antiquities of Athens and Other Monuments of Greece (London). Reprint 2007, New York: Princeton Architectural Press.

Sutherland, I. E. (1963). Sketchpad: A Man-Machine Graphical Communication System. Technical Report No. 296, Lincoln Laboratory, Massachusetts Institute of Technology Retrieved via: Defence Technical Information Center (stinet.dtic.mil). http://handle.dtic.mil/100.2/AD404549

Sutherland, I.E. (1974). Three Dimensional Data Input by Tablet. Proc. IEEE 62(4).

Swinson, P. (1982). Logic Programming: A computing tool for the architect of the future. ComputerAided Design, 14(2), pp. 97-104.

Tansey, R.G., Kleiner, F.S. (1996). Gardner's Art Through the Ages. 10th ed. Fort Worth: Harcourt Brace College Publishers. 
Talašová, Z., Achten, H. (2009). Fuzzy Rule Bases as a Tool of the History of Architecture: Application to the Analysis of Villas Designed by Adolf Loos. Computation: The New Realm of Architectural Design; eCAADe 2009 Conference Proceedings, pp. 705-712.

Toth, B., Salim F.D., Drogemuller, R., Frazer, J.H., Burry, J. (2011). Closing the loop of design and analysis: Parametric modelling tools for early decision support. CAADRIA 2011 Conference Proceedings, Newcastle (Australia) , pp. 525-534.

Turkle, S. (2009). The View From the 1980's Chapter in Simulation and its discontents, Turkle, S., Clancey, W.J., Helmreich, S., Loukissas, Y.A., Myers, N. Cambridge, Mass.: MIT press. pp. 9-42.

Terzidis, K. (2004). Algorithmic Design: A Paradigm Shift in Architecture? Architecture in the Network Society; eCAADe 2004 Conference Proceedings, Copenhagen (Denmark), pp. 201-207.

Ullrich T., Fellner, D.W.(2007). Robust shape fitting and semantic enrichment. International Symposium of the International Committee for Architectural Photogrammetry Conference Proceedings (v21).

Van Mersbergen, A.M.(1998). Rhetorical Prototypes in Architecture: Measuring the Acropolis. Philosophical Polemic Communication Quarterly, Vol. 46, 1998.

Vitruvius Pollio, M. (15BC). De architectura. as D. Rowland - T.N. Howe (1999) : Vitruvius. Ten Books on Architecture. Cambridge University Press.

Weisstein, E.W. (2011). Parametric Equations. From MathWorld : A Wolfram Web Resource. Retreived from: http://mathworld.wolfram.com/ParametricEquations.html

Wong, C.K.D., Cheung, Y.F.L. (2010). Simply complex: a case study of construction-driven design using computational methods. CAADRIA 2010 Conference Proceedings, Hong Kong, pp. 585-594.

Woodbury, R., Aish, R., Kilian, A. (2007a). Some patterns for parametric modelling. ACADIA 2007 Conference Proceedings, Halifax (Nova Scotia), pp. 222-229.

Woodbury, R., Marques, D. (2007b). Managing Contingency in Parametric Models through Implicit Relational Modelling. CAADFutures 2007 Conference Proceedings, Sydney (Australia), pp. 279-288.

Woodbury, R., Qian, C.Z., Chen, V.Y. (2007c). Participant Observation Can Discover Design Patterns in Parametric Modeling. ACADIA 2007 Conference Proceedings, Halifax (Nova Scotia), pp. $230-241$.

Woodbury, R. (2010). Elements of Parametric Design. London, NY: Routledge.

Zambas, C. (2002). The Refinements of the Columns of the Parthenon. Athens: YSMA.

\section{Web references and links}

\section{CumInCAD database}

CumInCAD is a cumulative index of publications about computer aided architectural design. It includes bibliographic information about over 10,400 records from journals and conferences such as ACADIA, ASCAAD, CAADRIA, eCAADe, SiGraDi, CAAD futures, DDSS and others. All papers include full abstracts. Full texts, in PDF, of some 7,600 papers are also available. A separate index of some 1,500 papers in Spanish language are maintained.

http://cumincad.scix.net/

\section{ACADIA}

ACADIA was formed in the early 1980's for the purpose of facilitating communication and critical thinking regarding the use of computers in architecture, planning and building science. A particular focus is education and the software, hardware and pedagogy involved in education.

The organization is also committed to the research and development of computer aides that enhance design 
creativity, rather than simply production, and that aim at contributing to the construction of humane physical environments.

http://www.acadia.org/

\section{eCAADe}

eCAADe (Education and research in Computer Aided Architectural Design in Europe) is a non-profit making association of institutions and individuals with a common interest in promoting good practice and sharing information in relation to the use of computers in research and education in architecture and related professions. eCAADe was founded in 1983

http://www.ecaade.org/

\section{CAADRIA}

An association to foster Computer Aided Architectural Design in Asia (CAADRIA) was founded in 1996. The conference is held by and for people involved with computer aided design in architecture schools in the region. They are attended by over one hundred researchers, teachers, students and practitioners who are interested in advancing knowledge and understanding in tools and application of digital design methods in architecture. CAADRIA seeks to mentor interest and capacity to undertake research throughout Asia.

http://www.caadria.org/

\section{ASCAAD}

A society of those who teach and conduct research in computer-aided architectural design in schools of architecture in the Arab World Region. We accept members from all over the world. ASCAAD aims to facilitate communication and information exchange regarding the use of computers and information technology in architecture, planning and building science.

http://www.ascaad.org/

\section{SiGraDi}

The Iberoamerican Society of Digital Graphics (SIGRADI); architecture groups, planners, designers and artists associated with the NEW meet as an annual conference, which discusses the latest Information and Communication Technologies applications and graphics capabilities, with the participation of relevant international specialists. The first Congress was held in 1997 SIGRADI in Buenos Aires. http://www.sigradi.org/

\section{CAADFutures}

CAADFutures was set up in 1985 with the purpose of promoting, through international conferences and publications, the advancement of Computer Aided Architectural Design in the service of those concerned with the quality of the built environment. The mission of the CAADFutures foundation is to promote research interactions and collaborations between researchers, including $\mathrm{PhD}$ students and their supervisors, and to provide a platform for communication among researchers in the of CAAD http://www.caadfutures.org/

\section{DDSS}

The International Conferences on Design \& Decision Support Systems in Architecture and Urban Planning are organised bi-annually by Eindhoven University of Technology. Traditionally, the DDSS conferences aim to be a platform for both starting and experienced researchers who focus on the development and application of computer support in the areas of urban planning and architectural design. This results in an interesting mix of well-established research projects and first explorations. It also leads to a very valuable cross-over of theories, methods, and technologies for support systems in the two different areas, architecture and urban planning.

http://www.ddss.nl/

\section{SmartGeometry}

Smartgeometry was founded in 2001 as a partnership between Practice, Research and Academia, formed by members of the world's leading architectural and engineering practices and educational institutions. 


\section{// Appendix}

Enlargements of figures and a few photos of final models from Section 2 follow. 

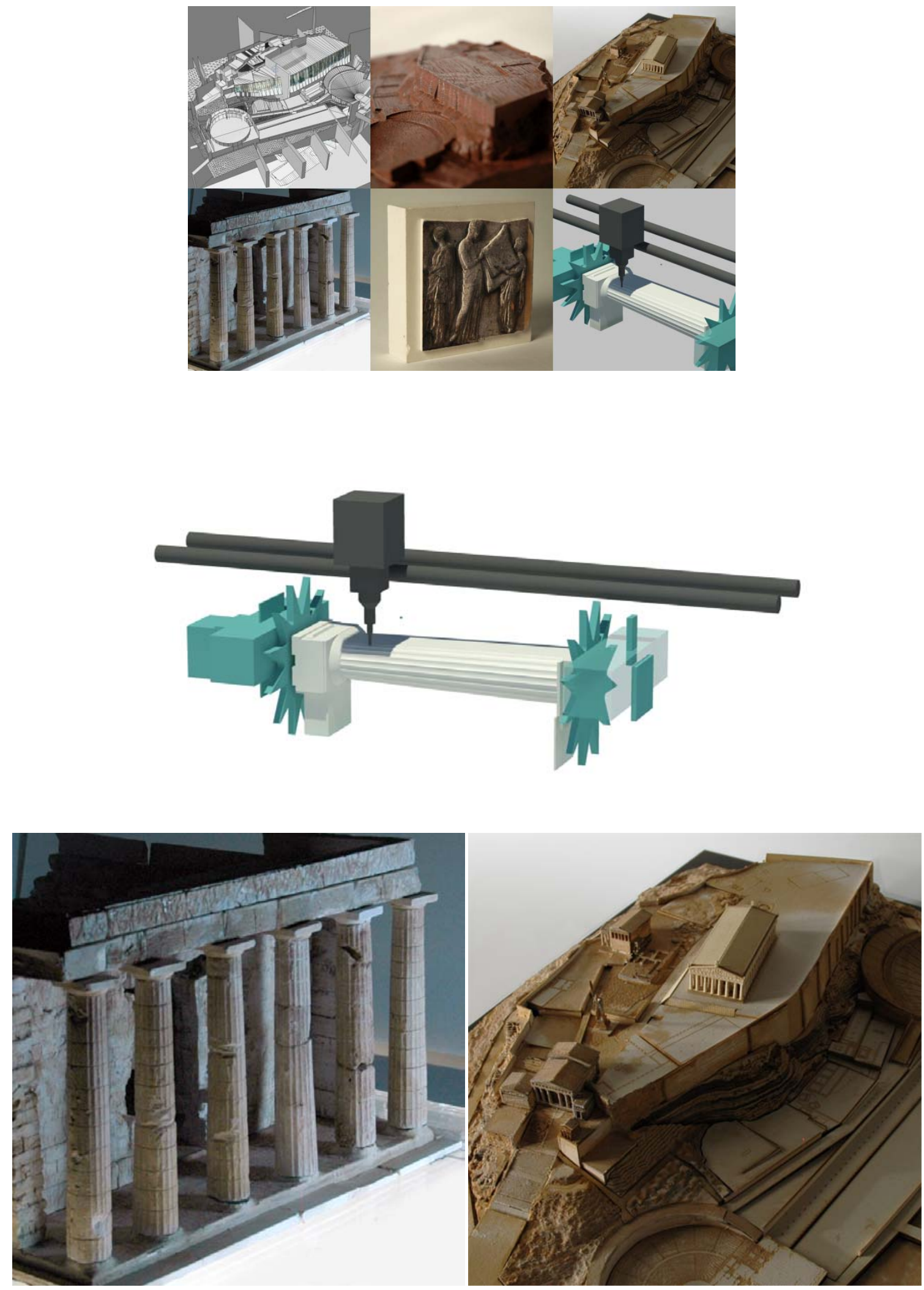

Enlargements of Figure 11; Experimentation prototypes for Educational Games project from large to small scale. 

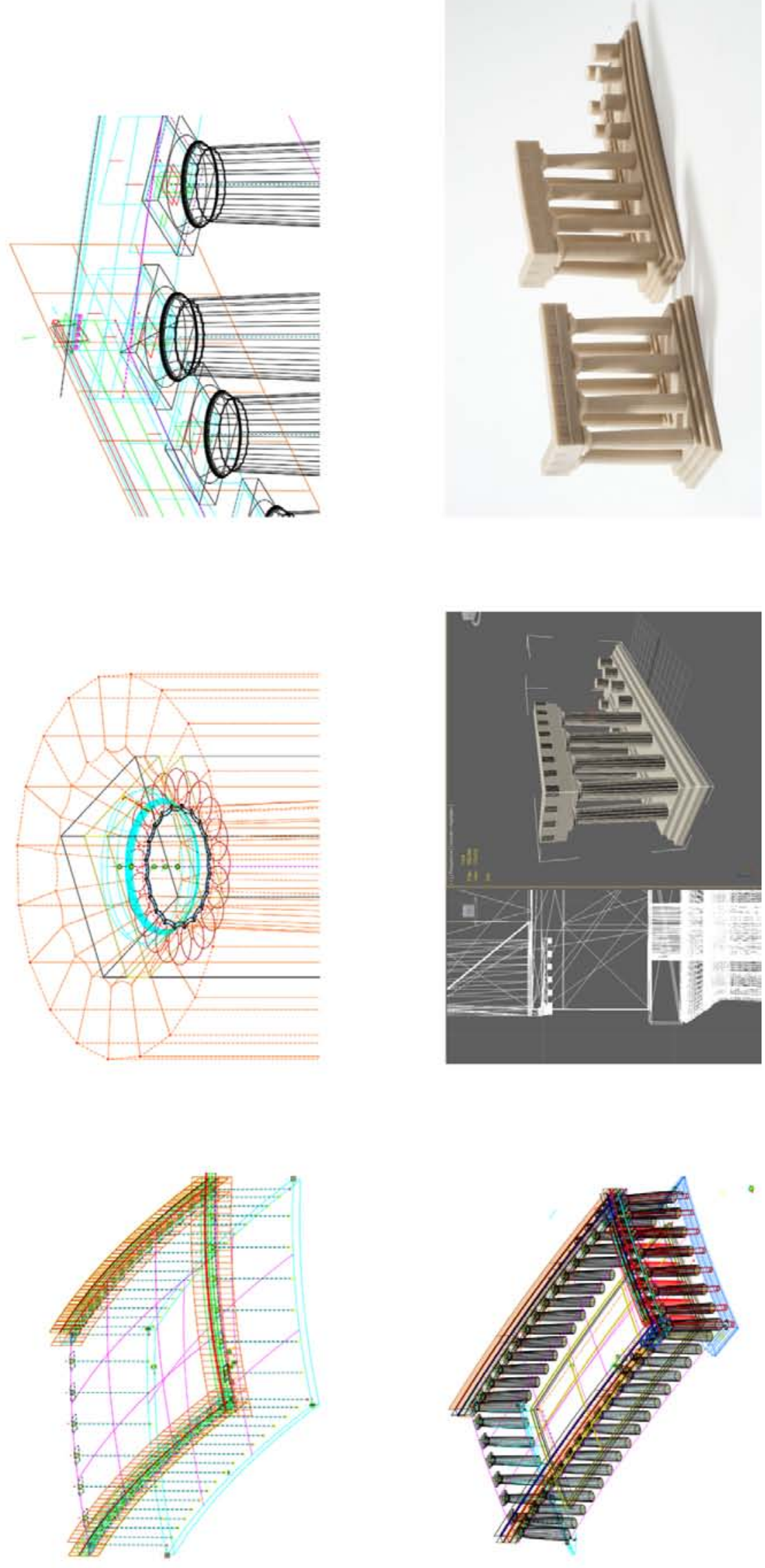

Enlargement of Figure 13; Project overview. 

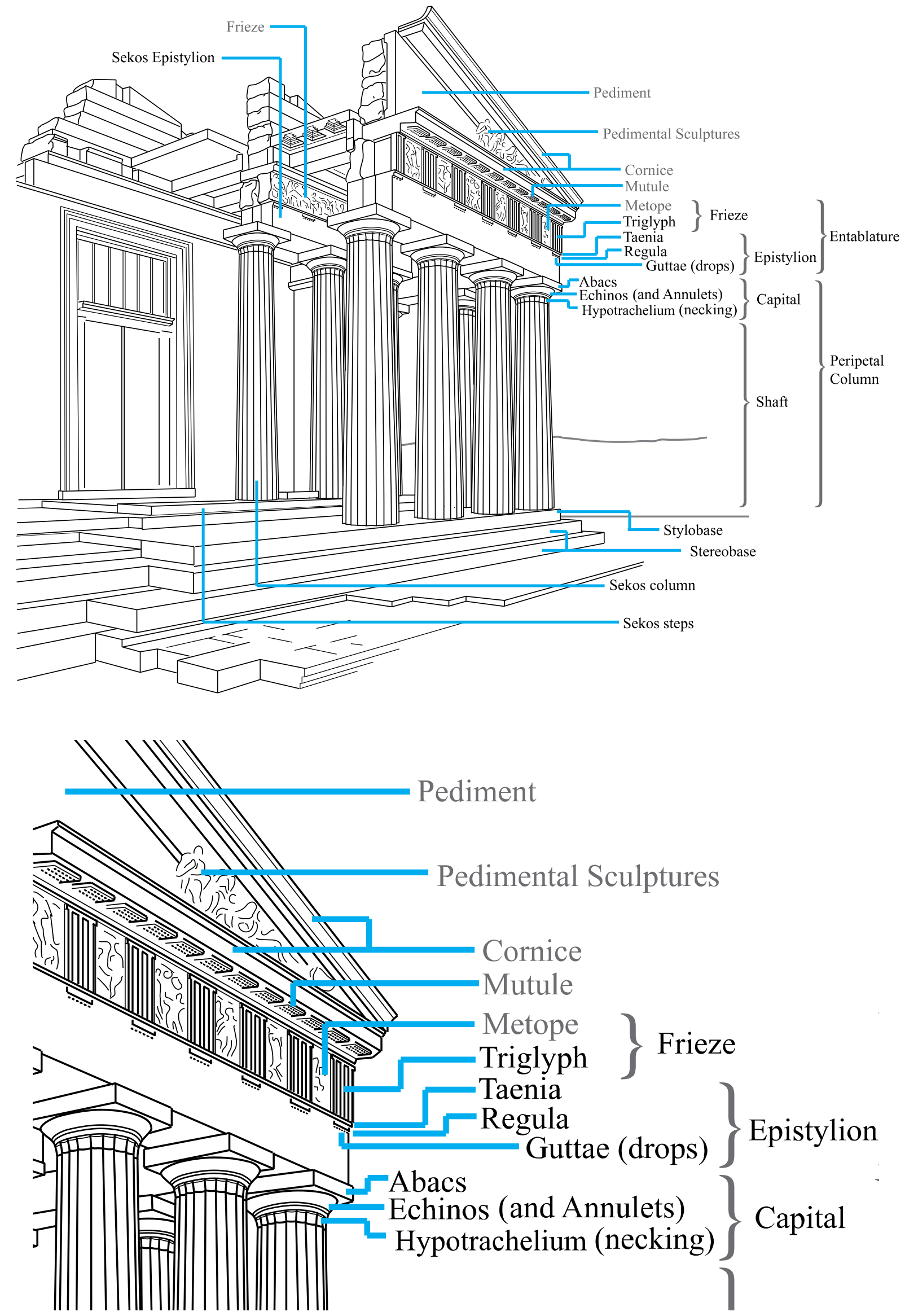

Enlargements of Figure 14; Parthenon anatomy after Neimann 


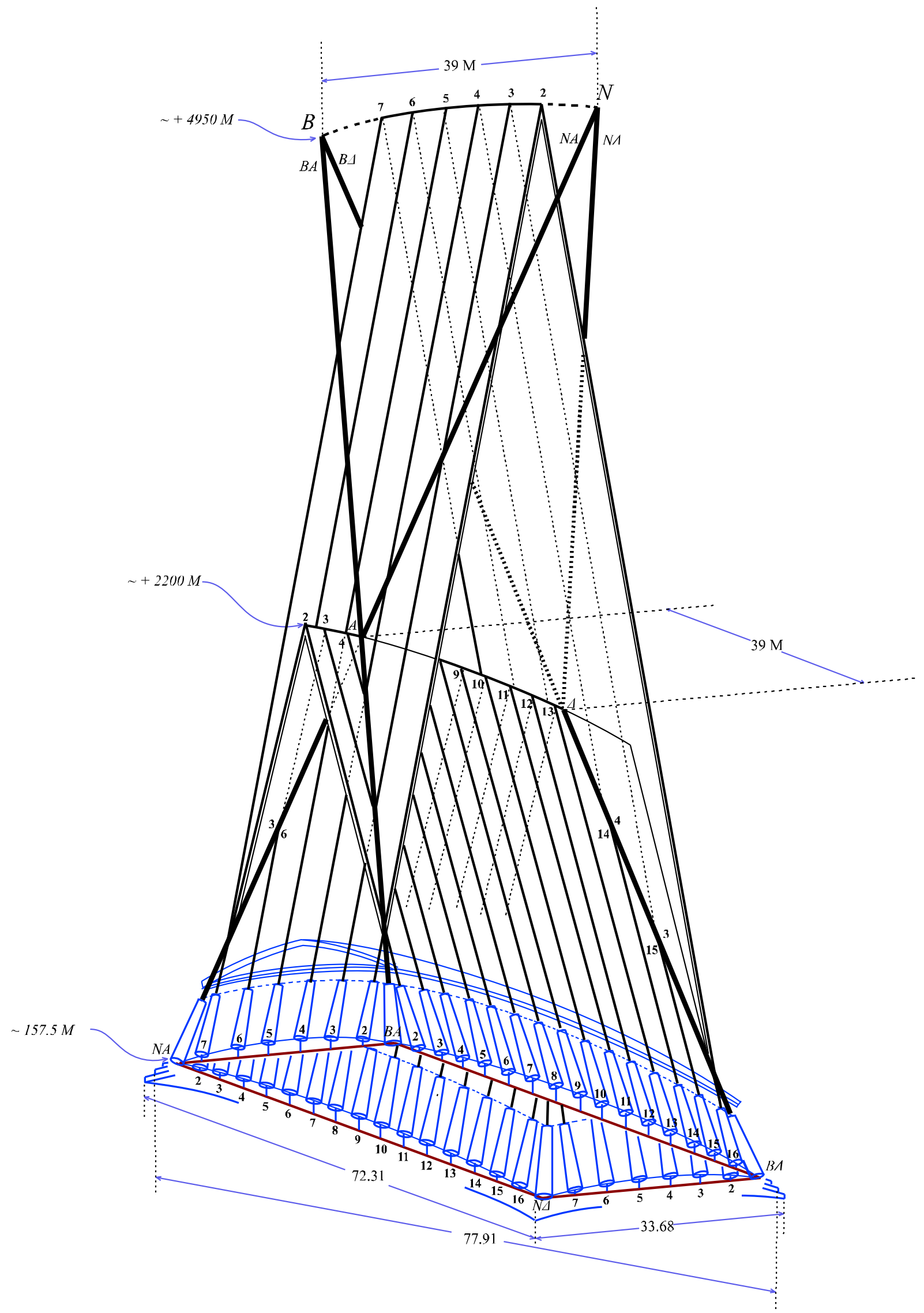

Enlargement of Figure 15; Parthenon hyper refinements after Korres 

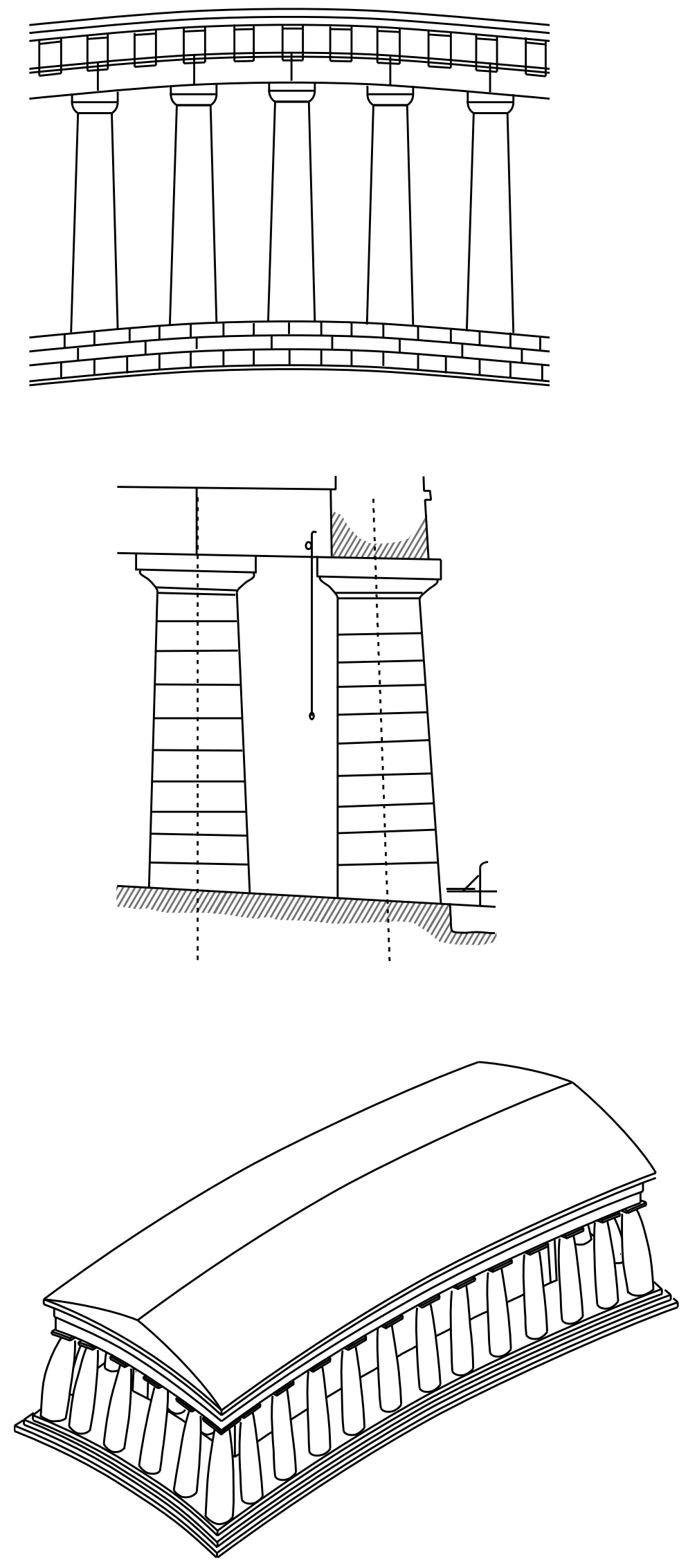

Enlargement of Figure 16; Hyper refinements of Greek temples, after Tansley and Kleiner, Dinsmoor, and Coulton. 


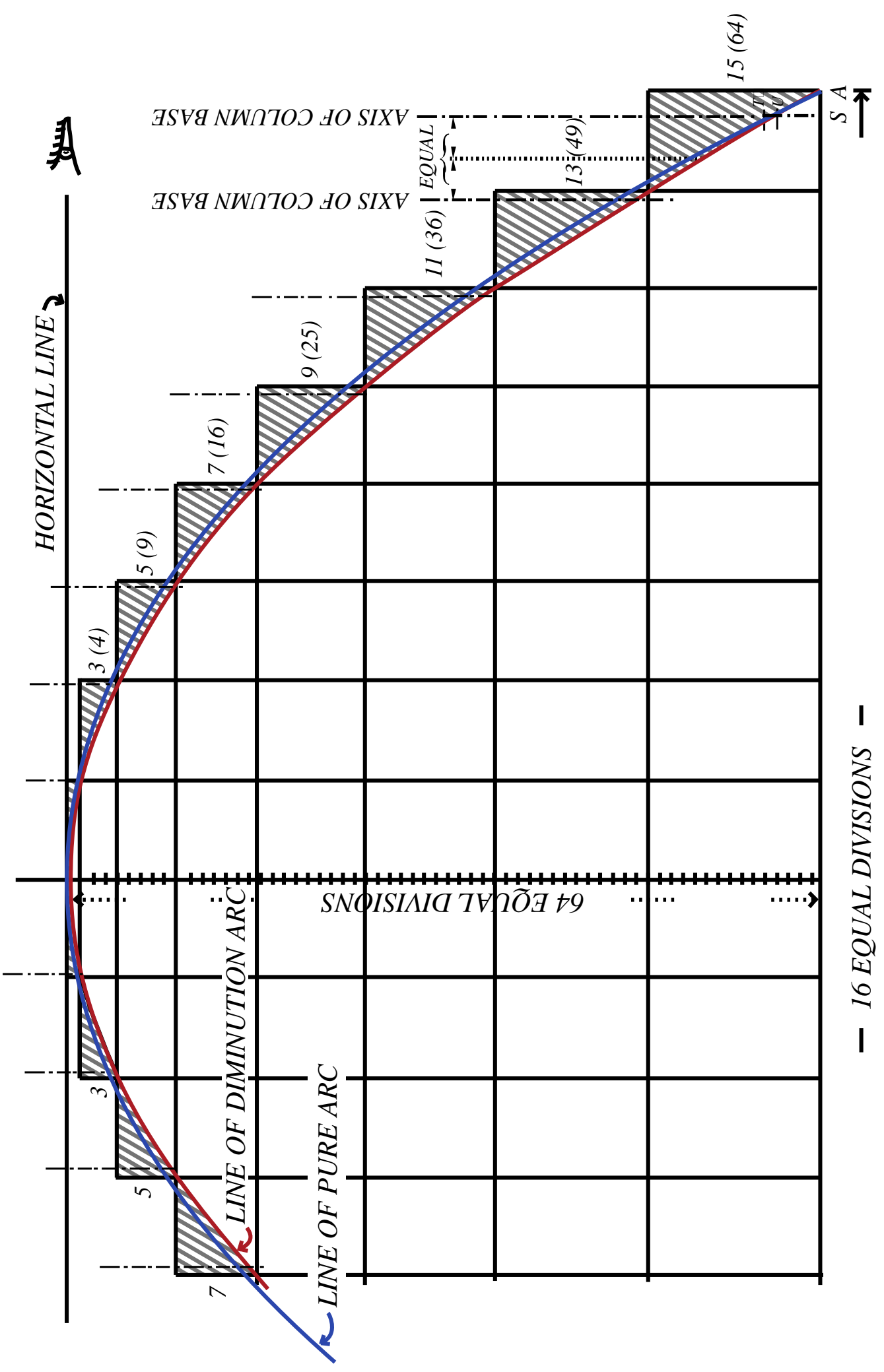

Enlargement of Figure 17; Diminution method in Greek temple Stylobase after Dinsmoor. Curve of diminution (red), Curve of true arc (blue) 

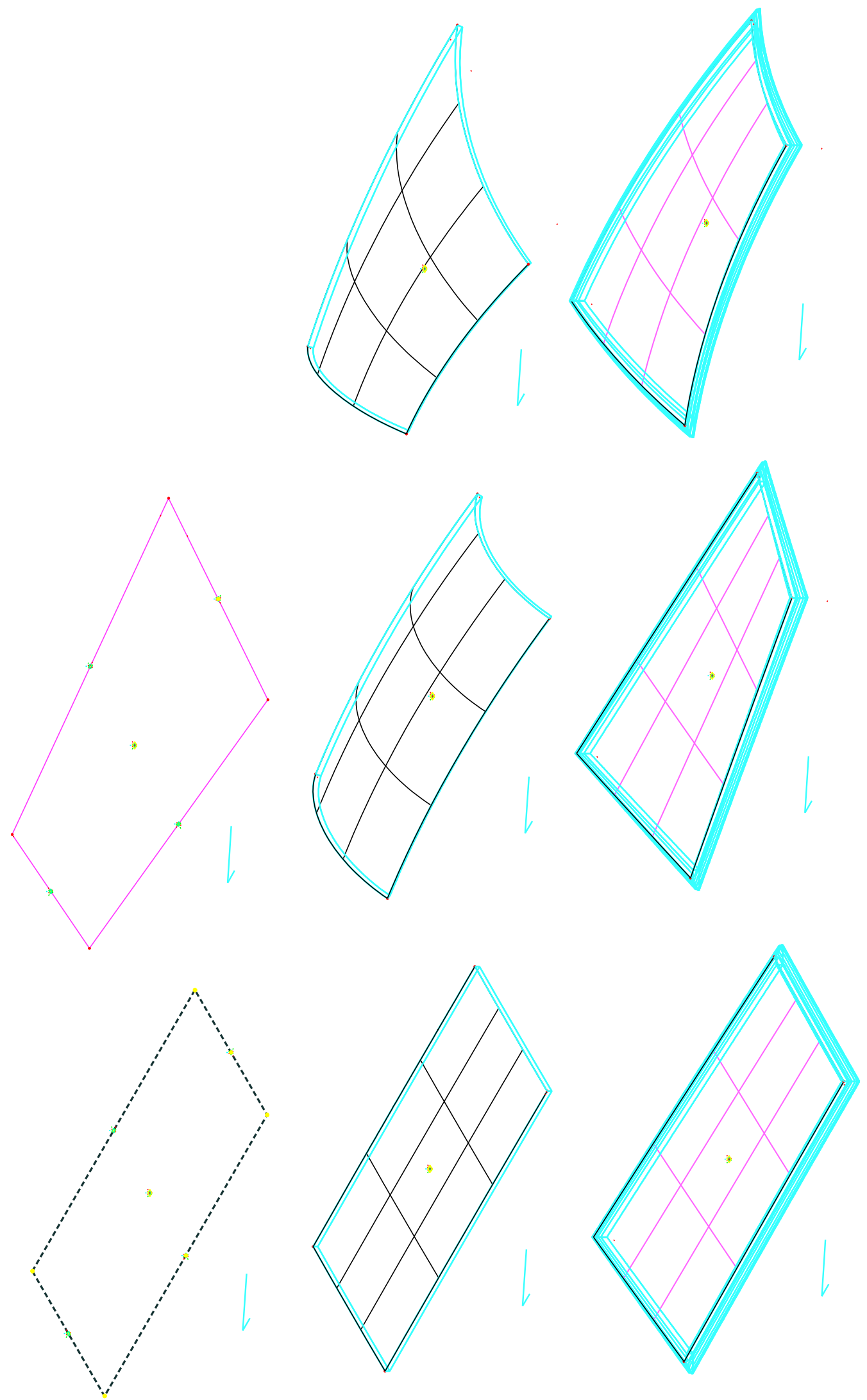

Enlargement of Figure 18; Stylobase setout and flexibility 

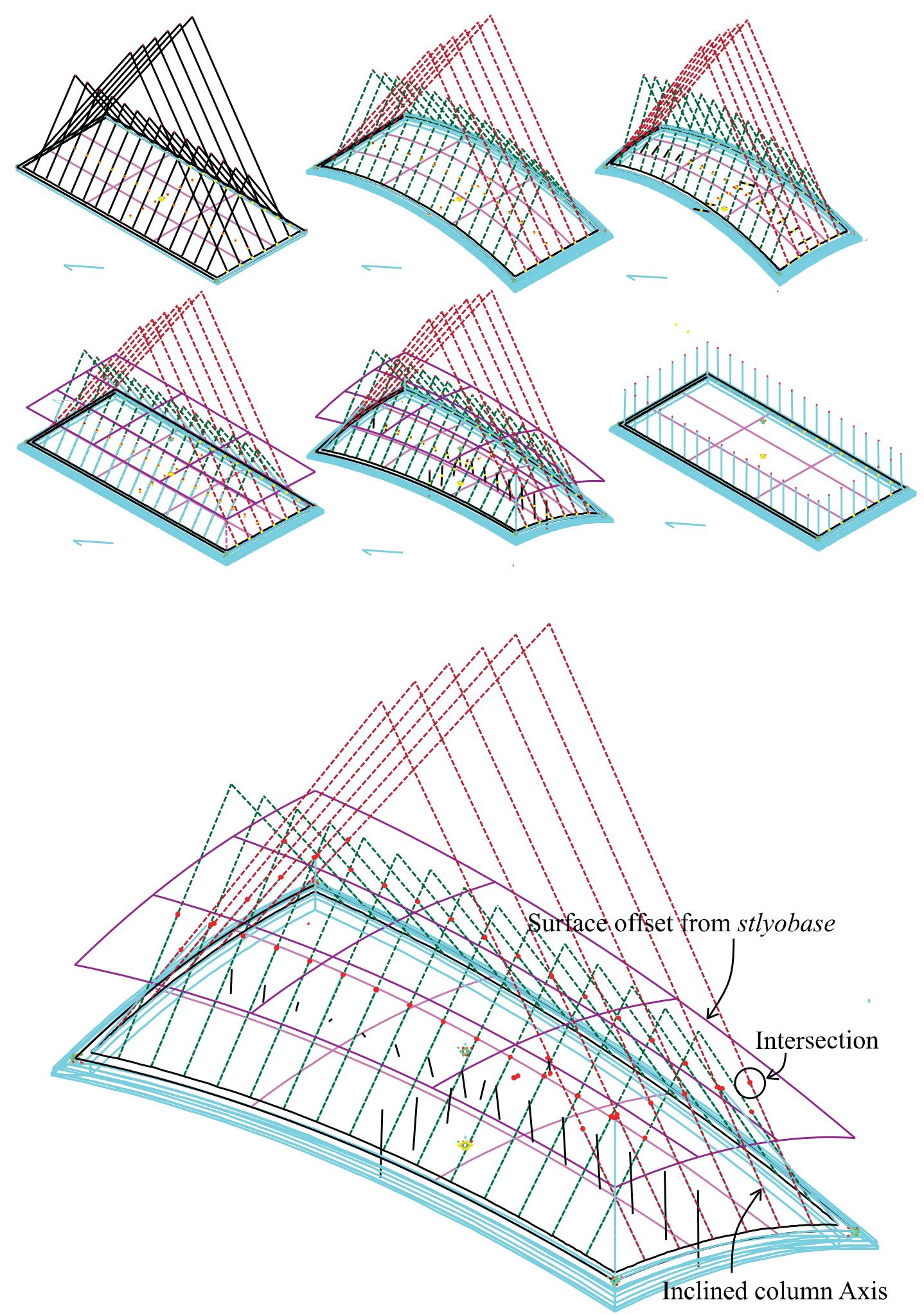

Enlargement of Figure 19; Column axis placement and incline and degrees of freedom. 


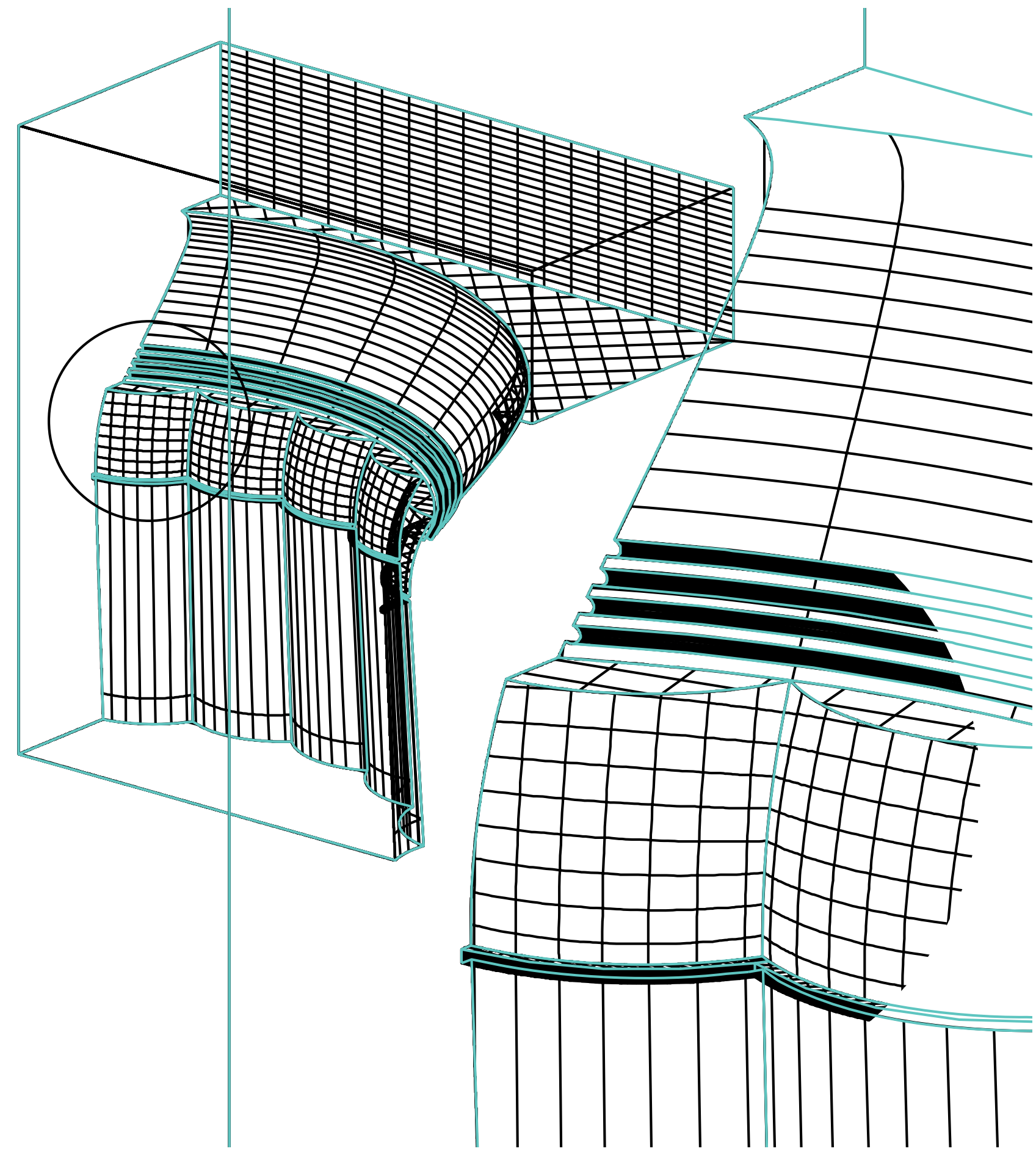

Enlargement of Figure 20; Illustration of problematic individual surfaces method of modelling a column. 

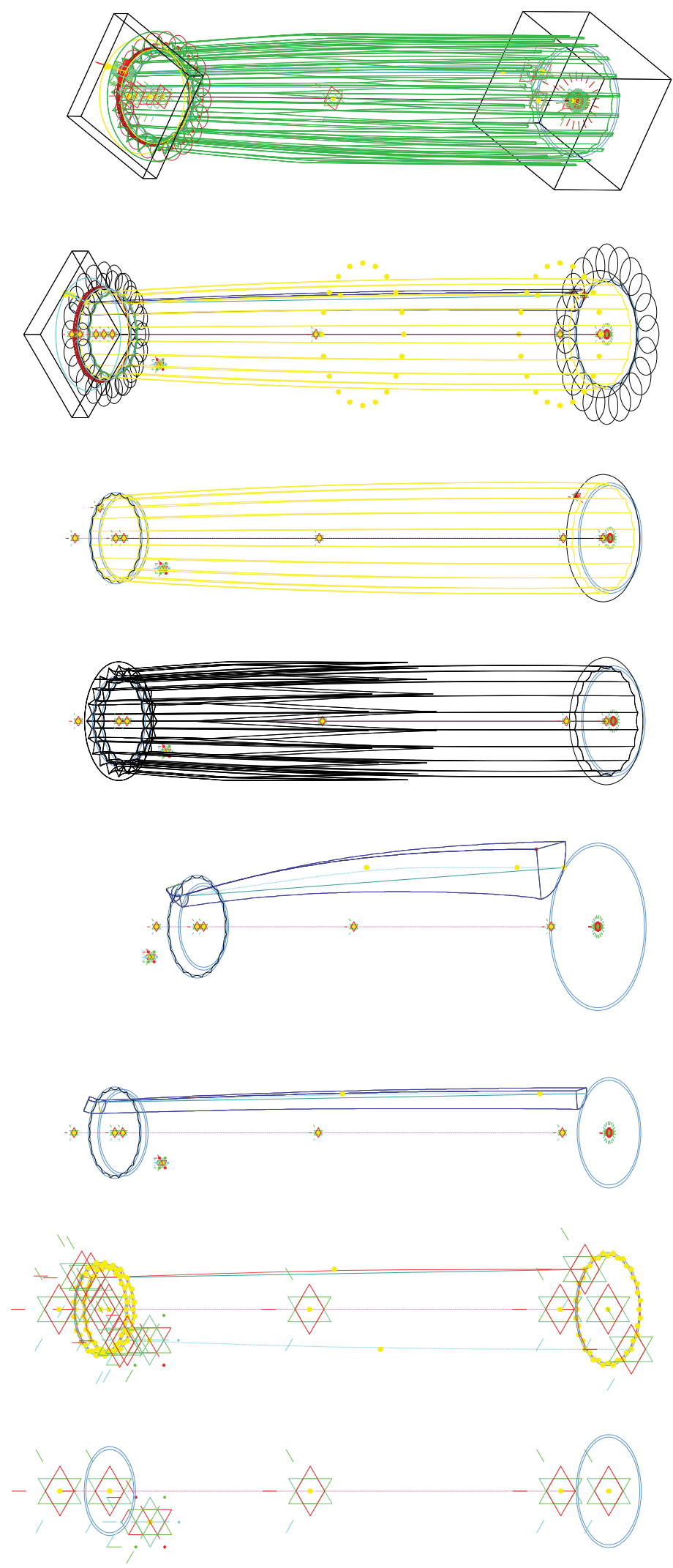

Enlargement of Figure 21; Column first iteration set 

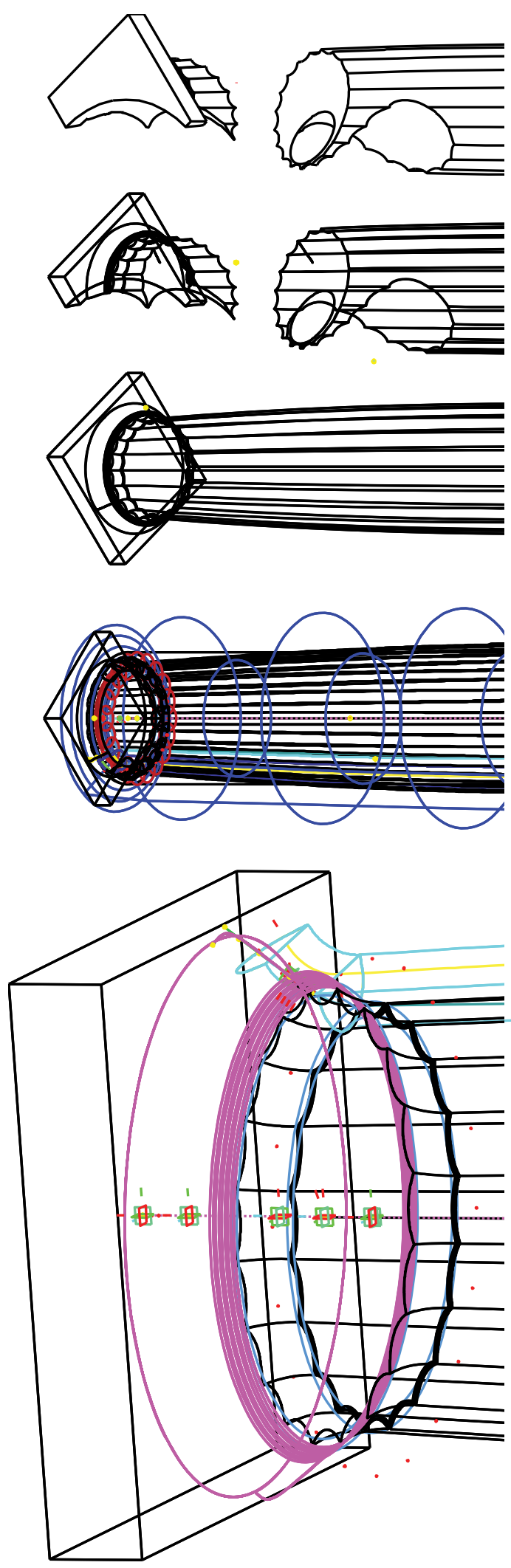

Enlargement of Figure 22; Model from initial solid column 

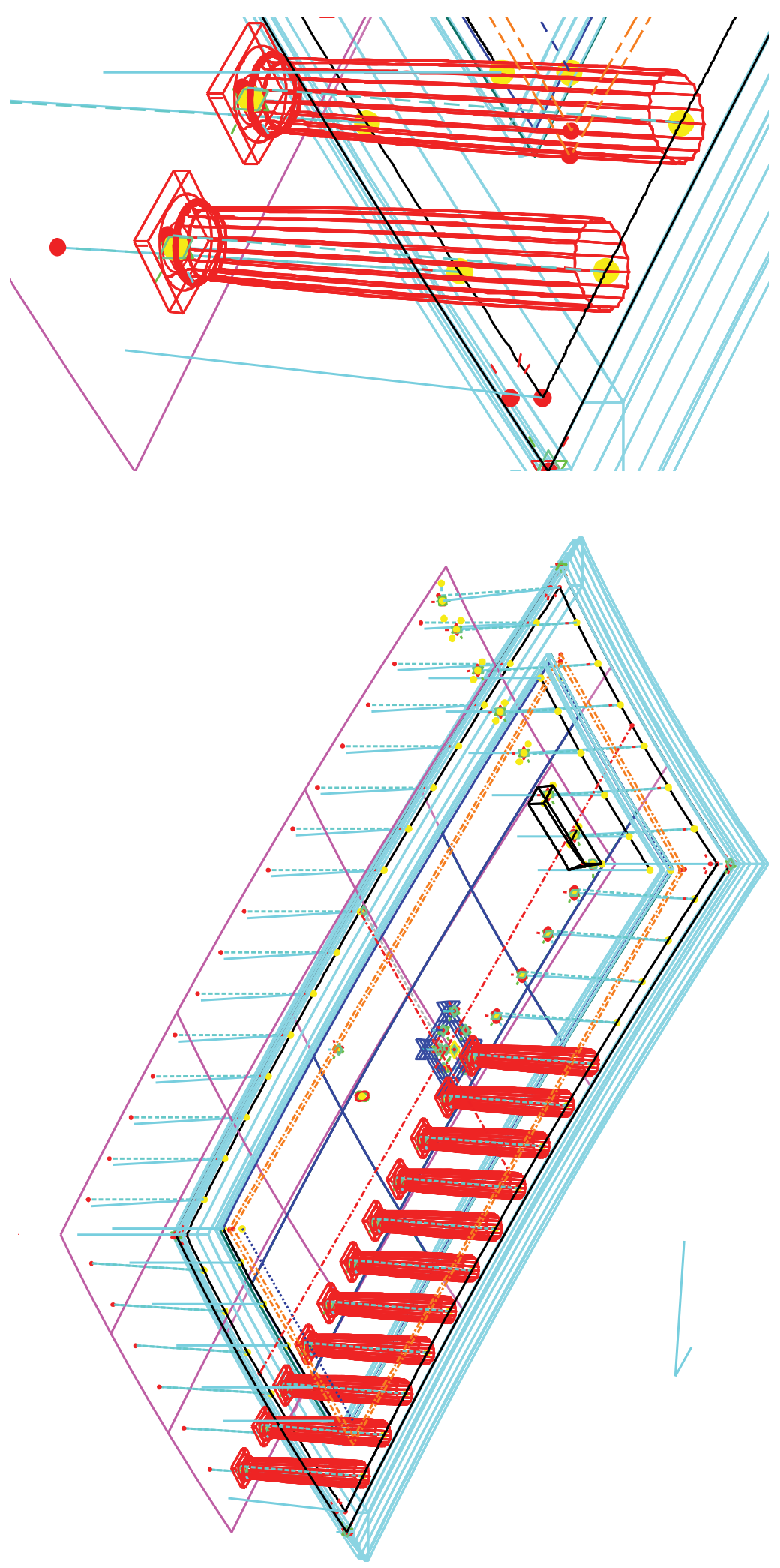

Enlargement of Figure 23; . ‘Unique column’ method; computationally expensive. 

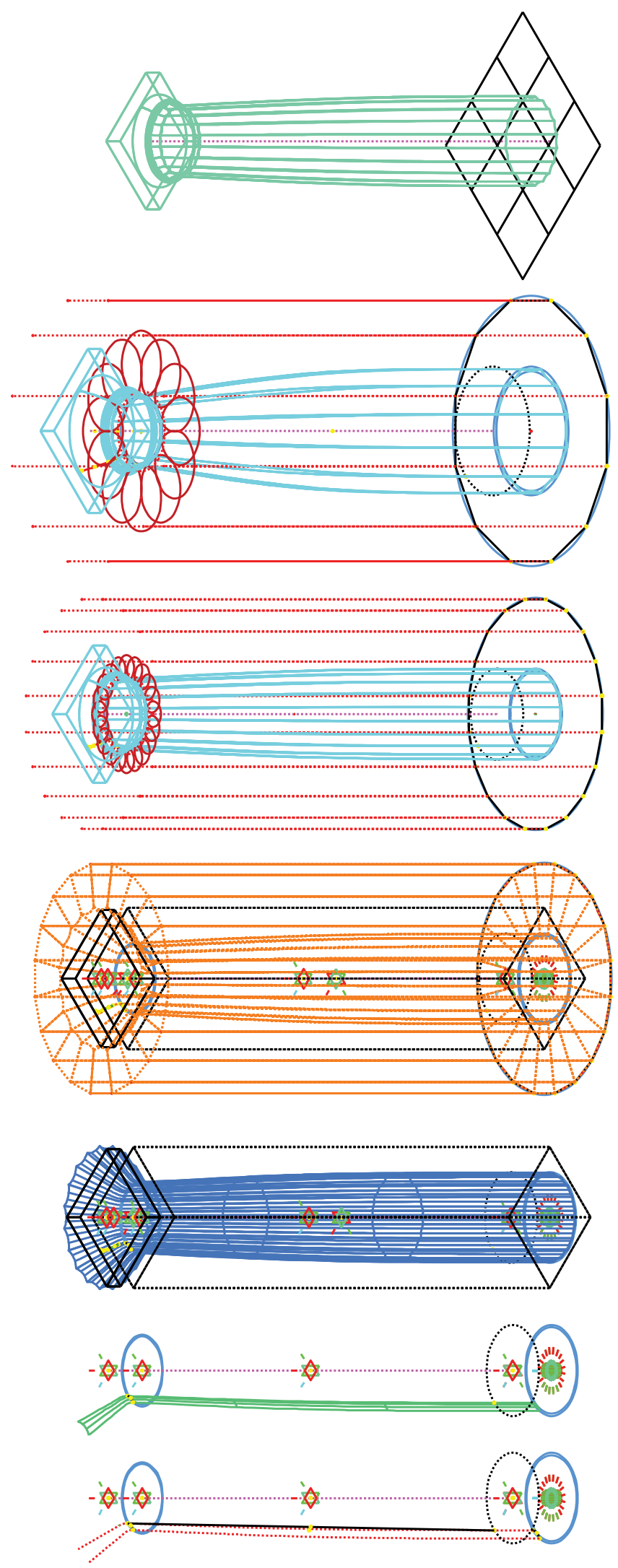

Enlargement of Figure 24; Final Column solid modelling sequence. 

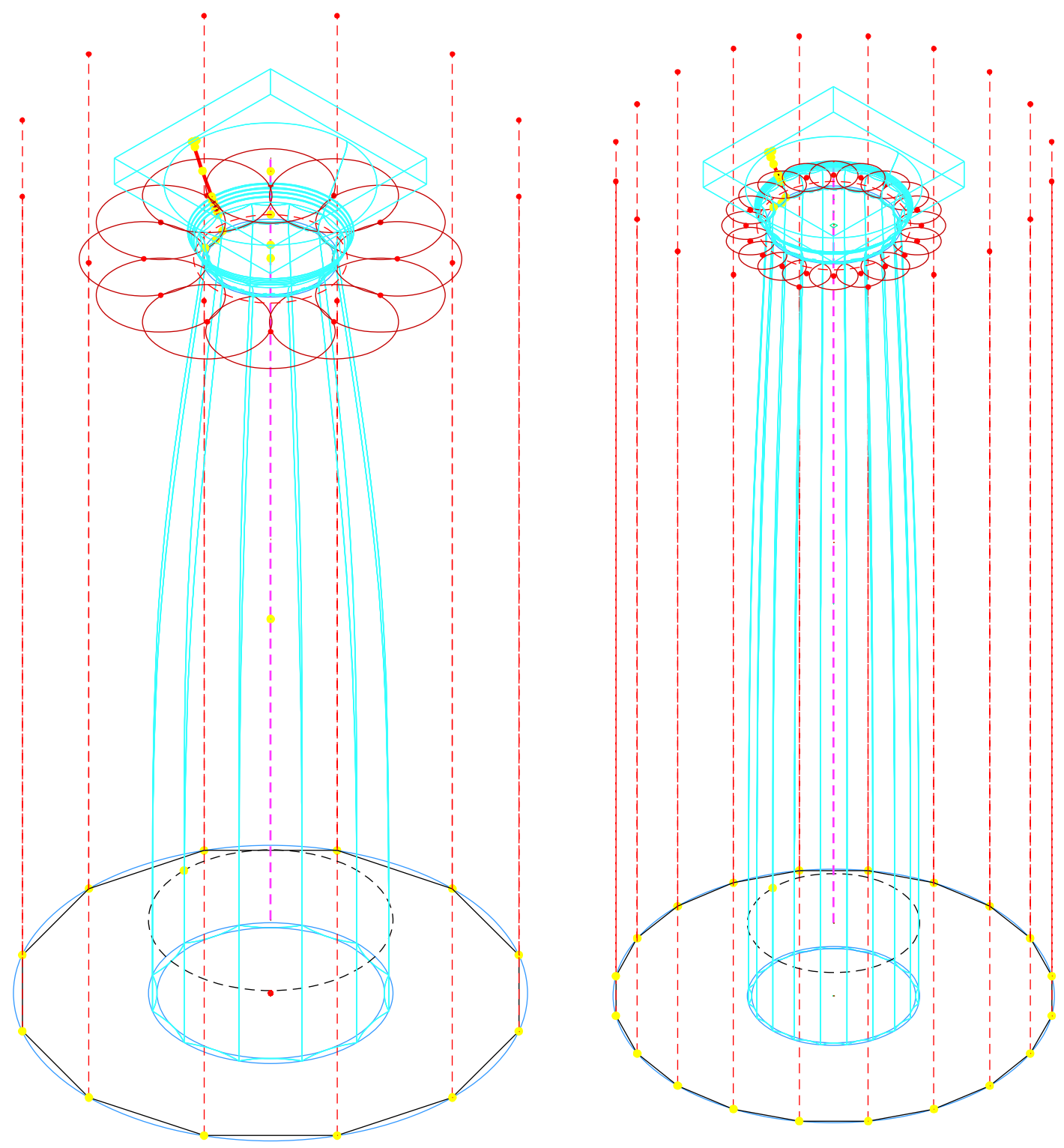

Enlargement of Figure 24; Variations of final Column solid modelling sequence. 


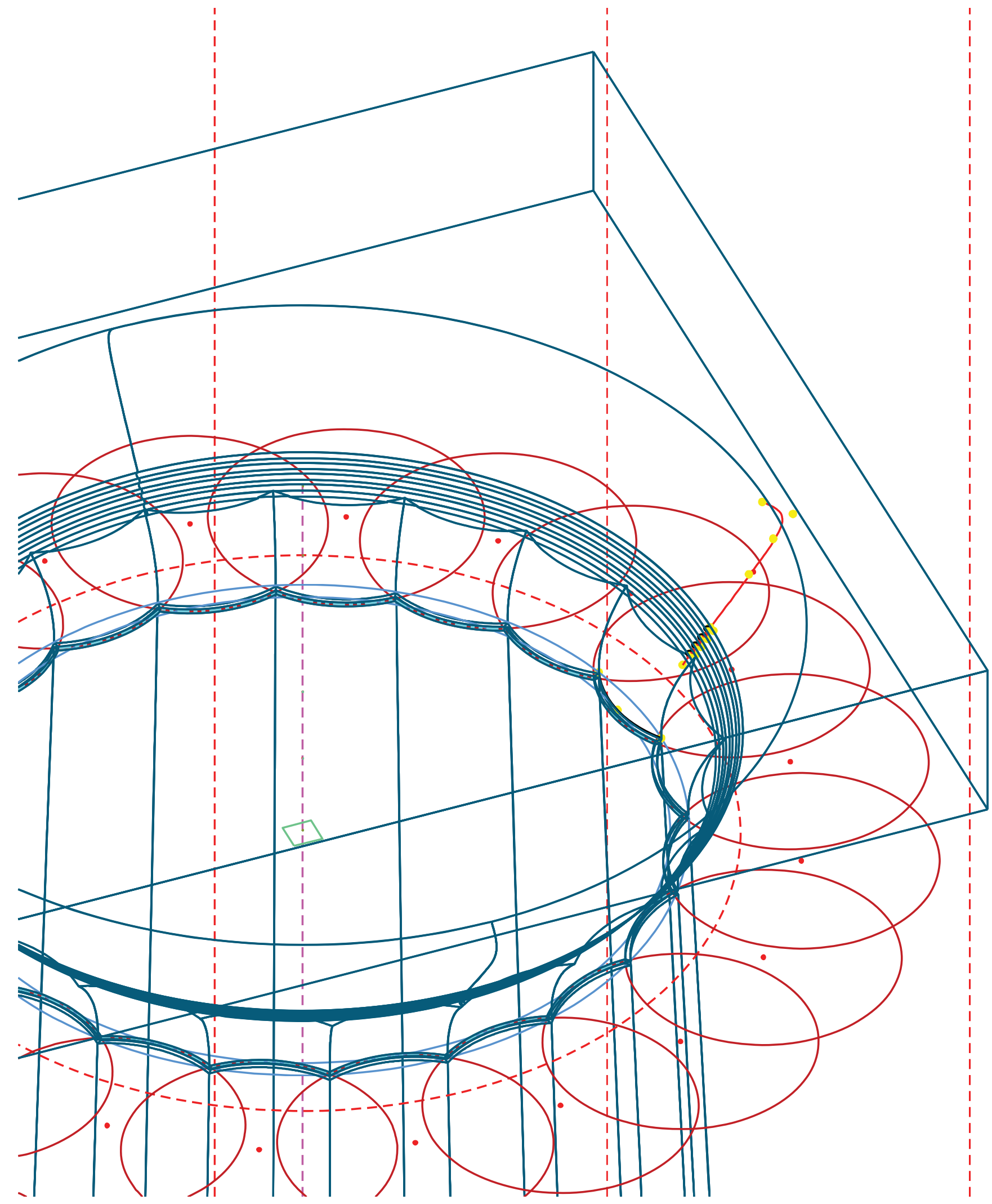

Additional view of final column model capital 


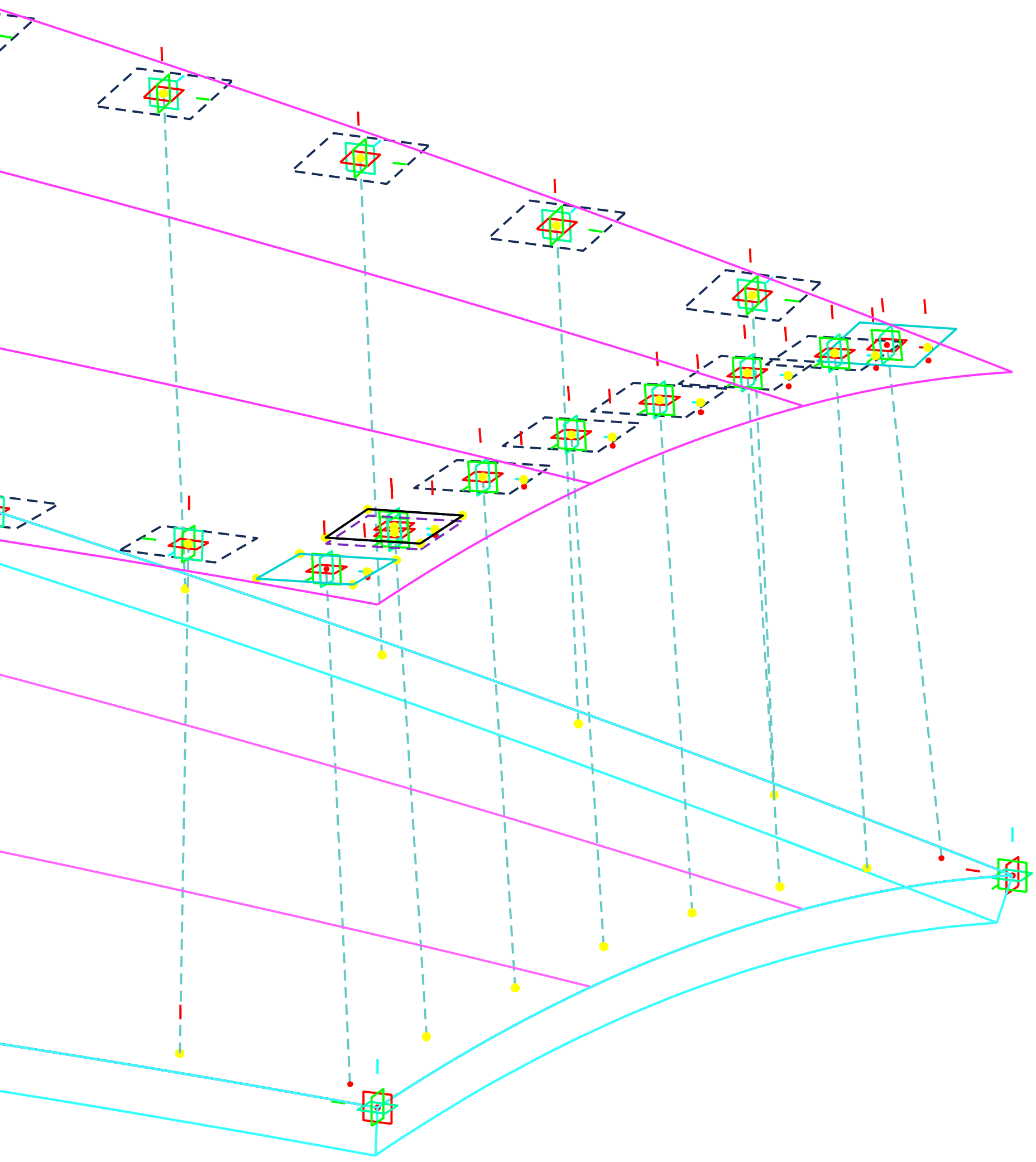

Enlargement of Figure 25; Epistylion modelling stage. 


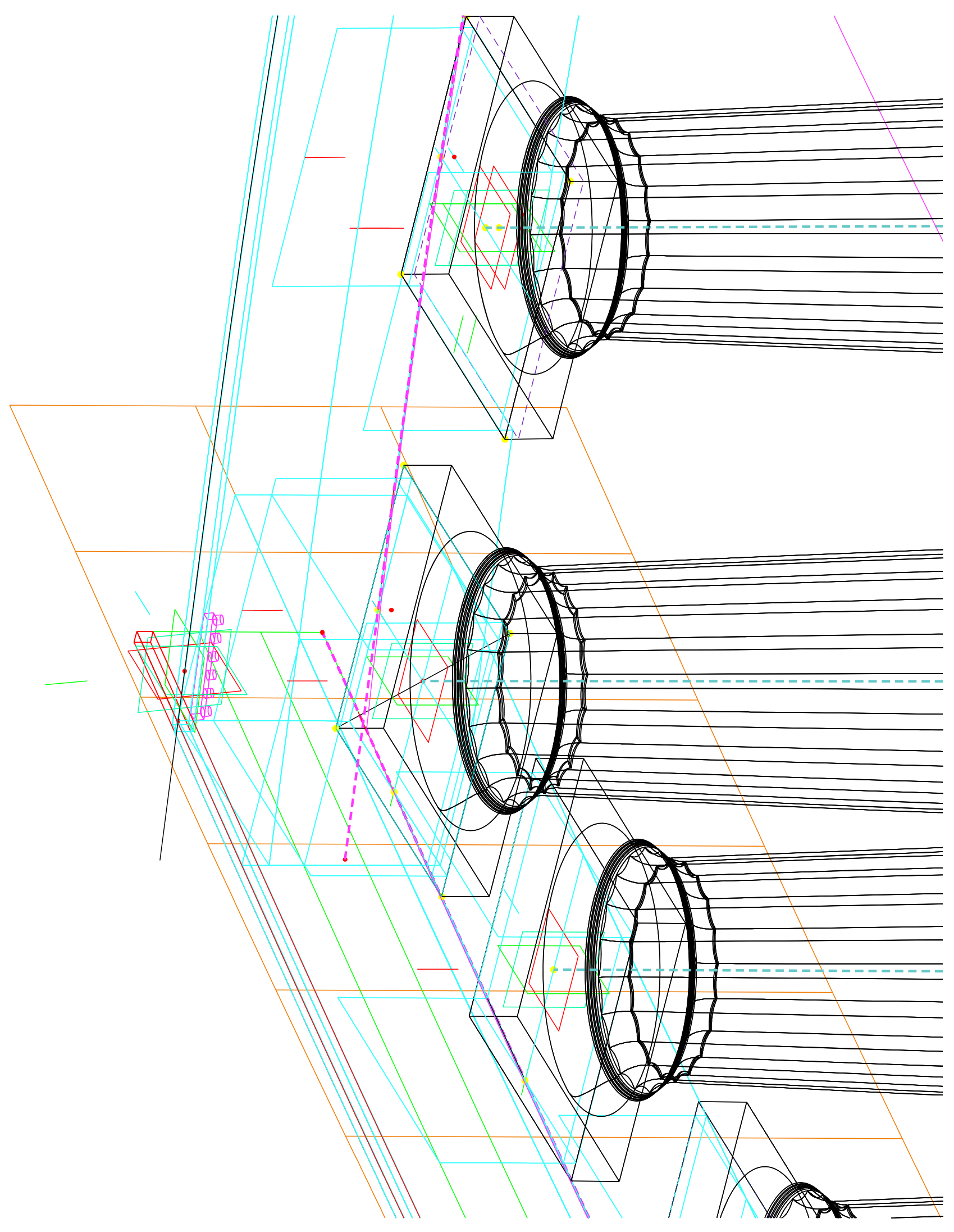

Enlargement of Figure 25; Epistylion modelling stage 


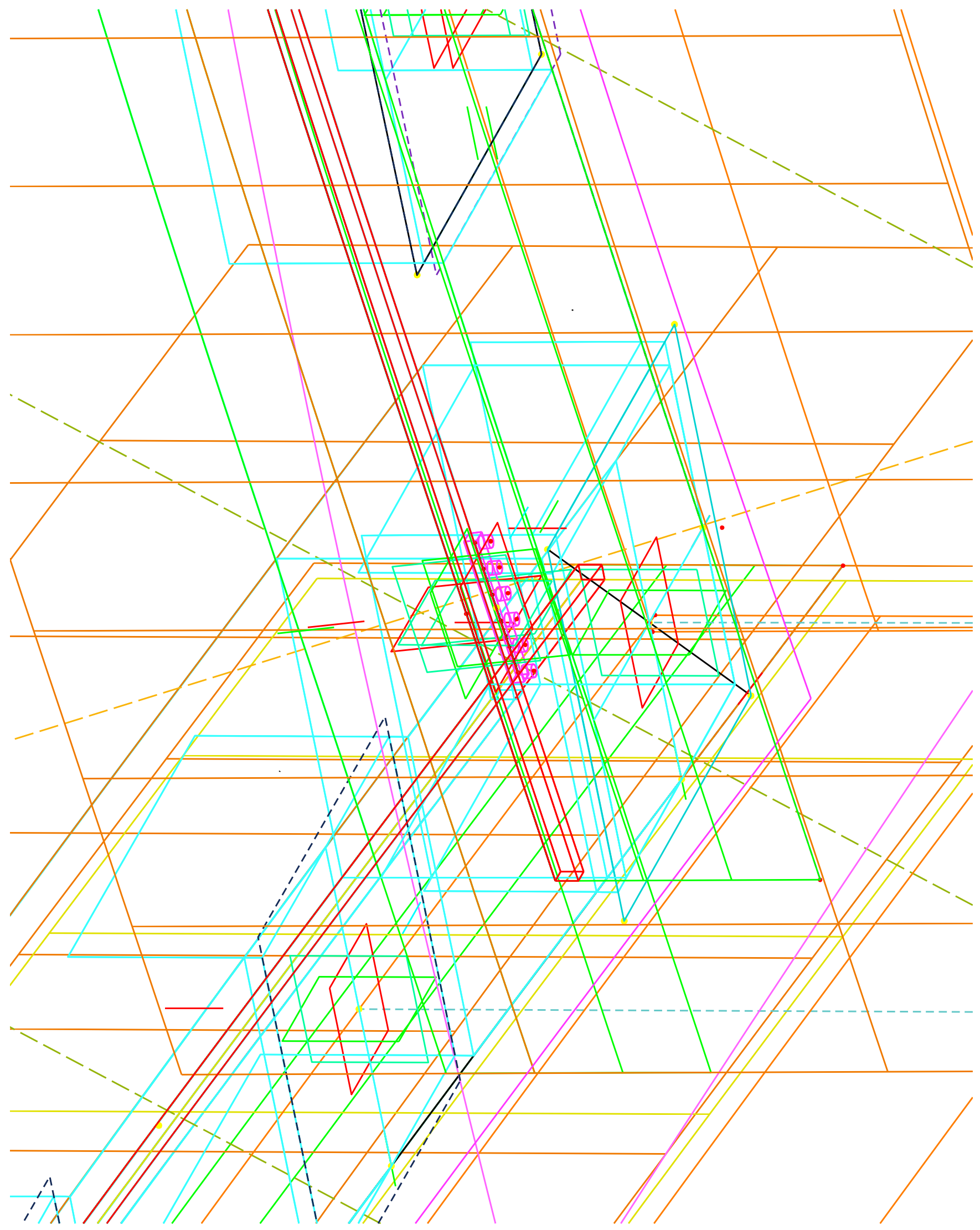

Additional view of Entablature modelling stage development. 


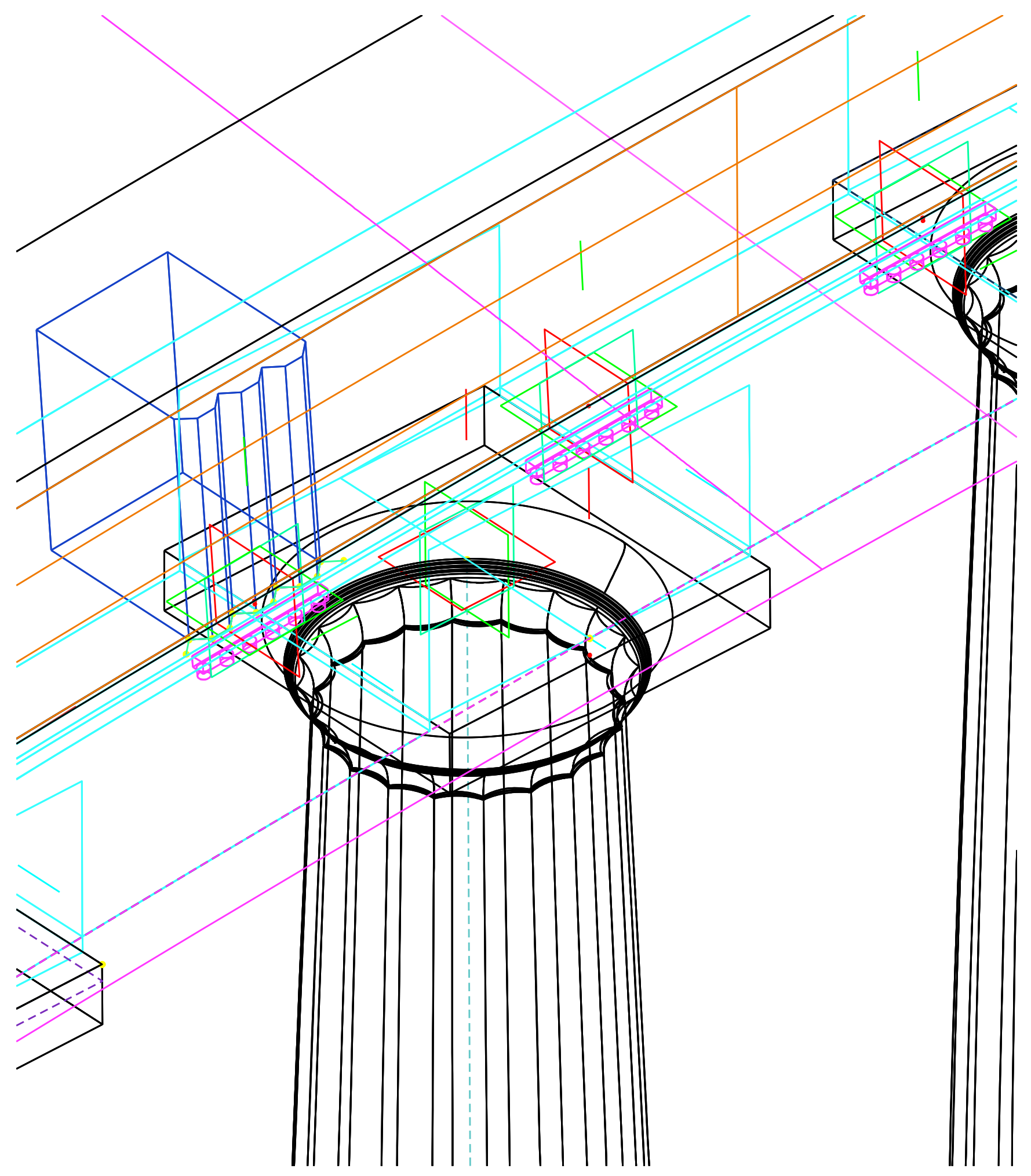

Enlargement of Figure 25; Epistylion modelling stage 


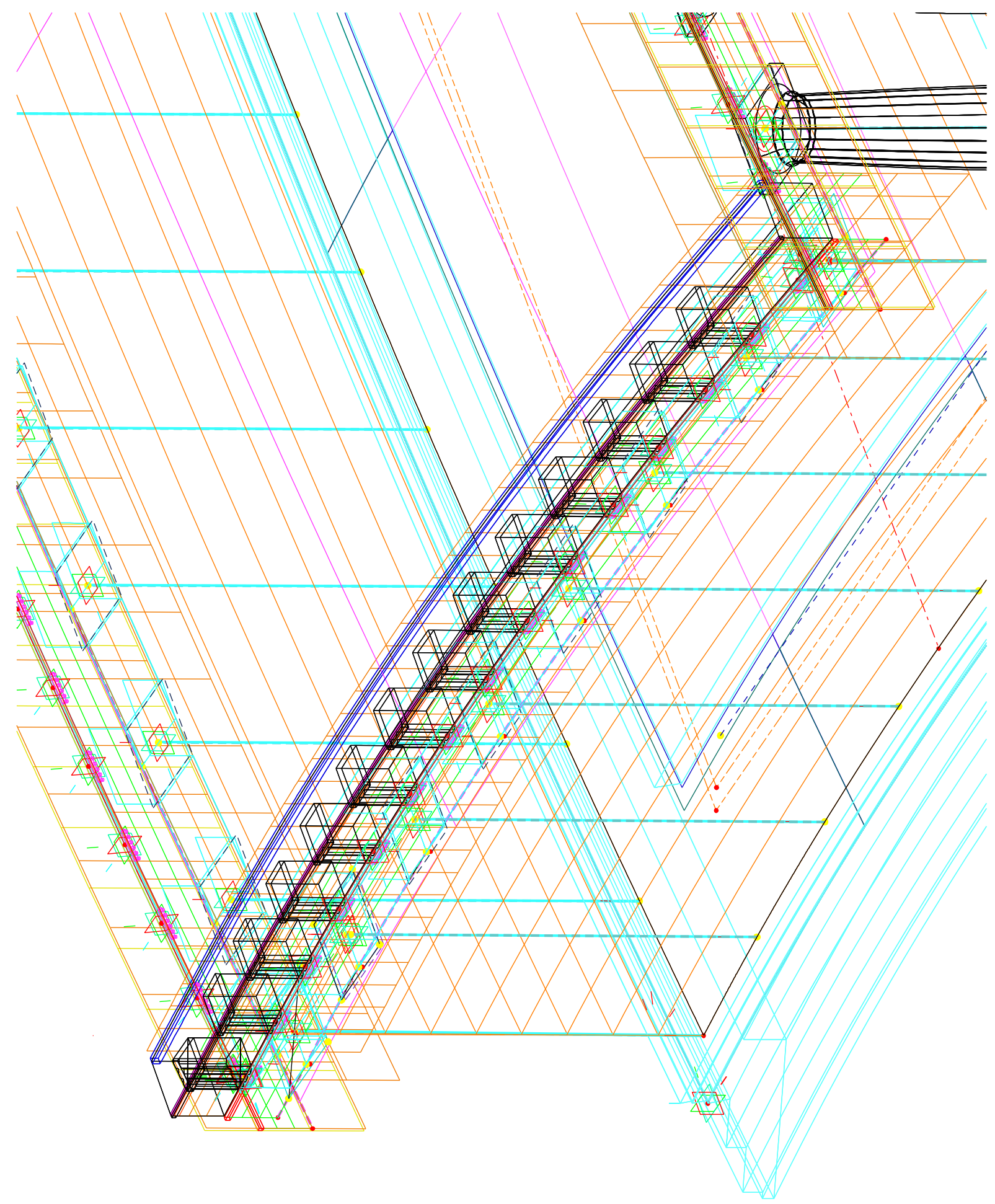

Enlargement of Figure 25; Epistylion modelling stage 


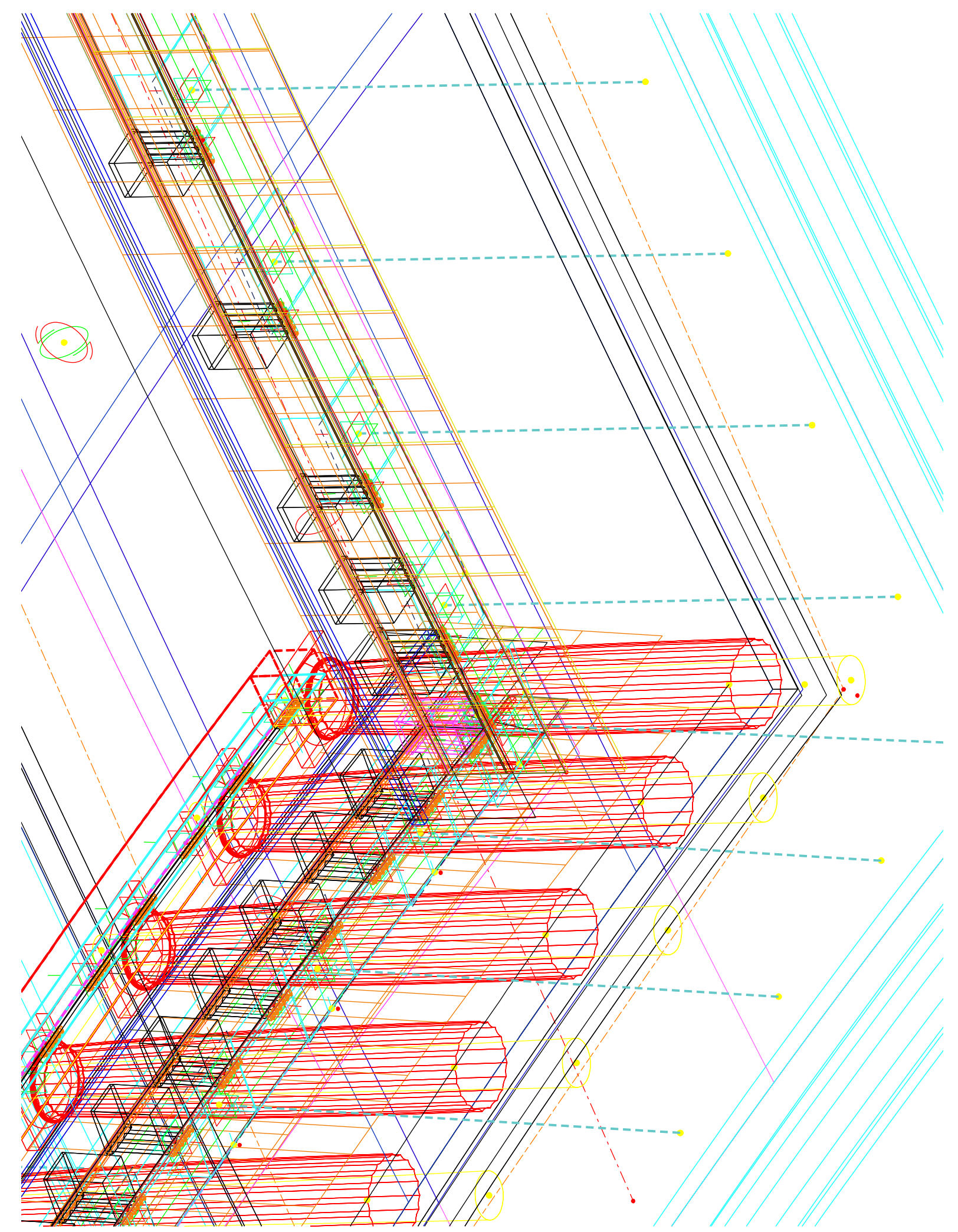

Additional view of Entablature modelling stage development. 


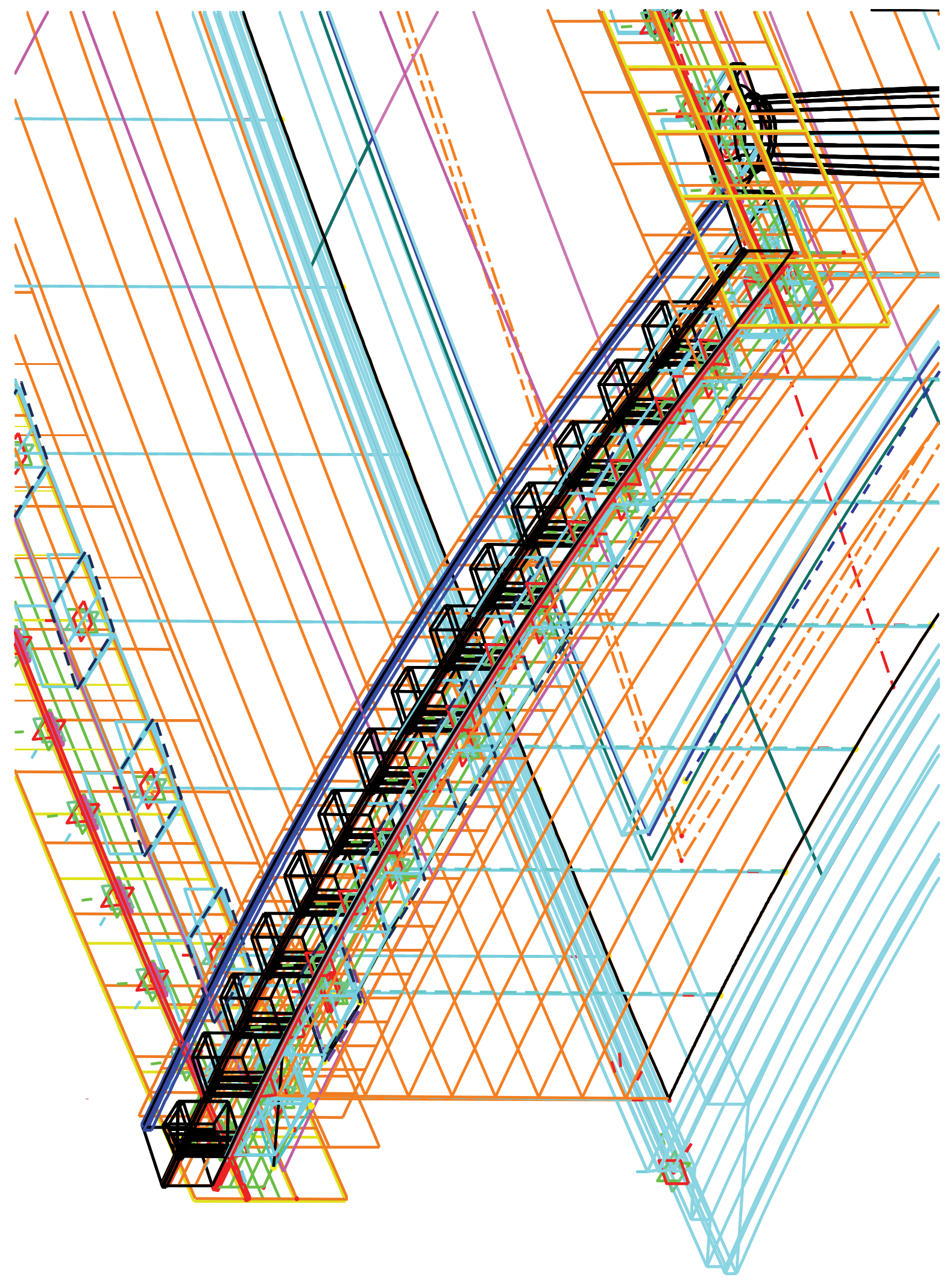

Additional view of Entablature modelling stage development. 


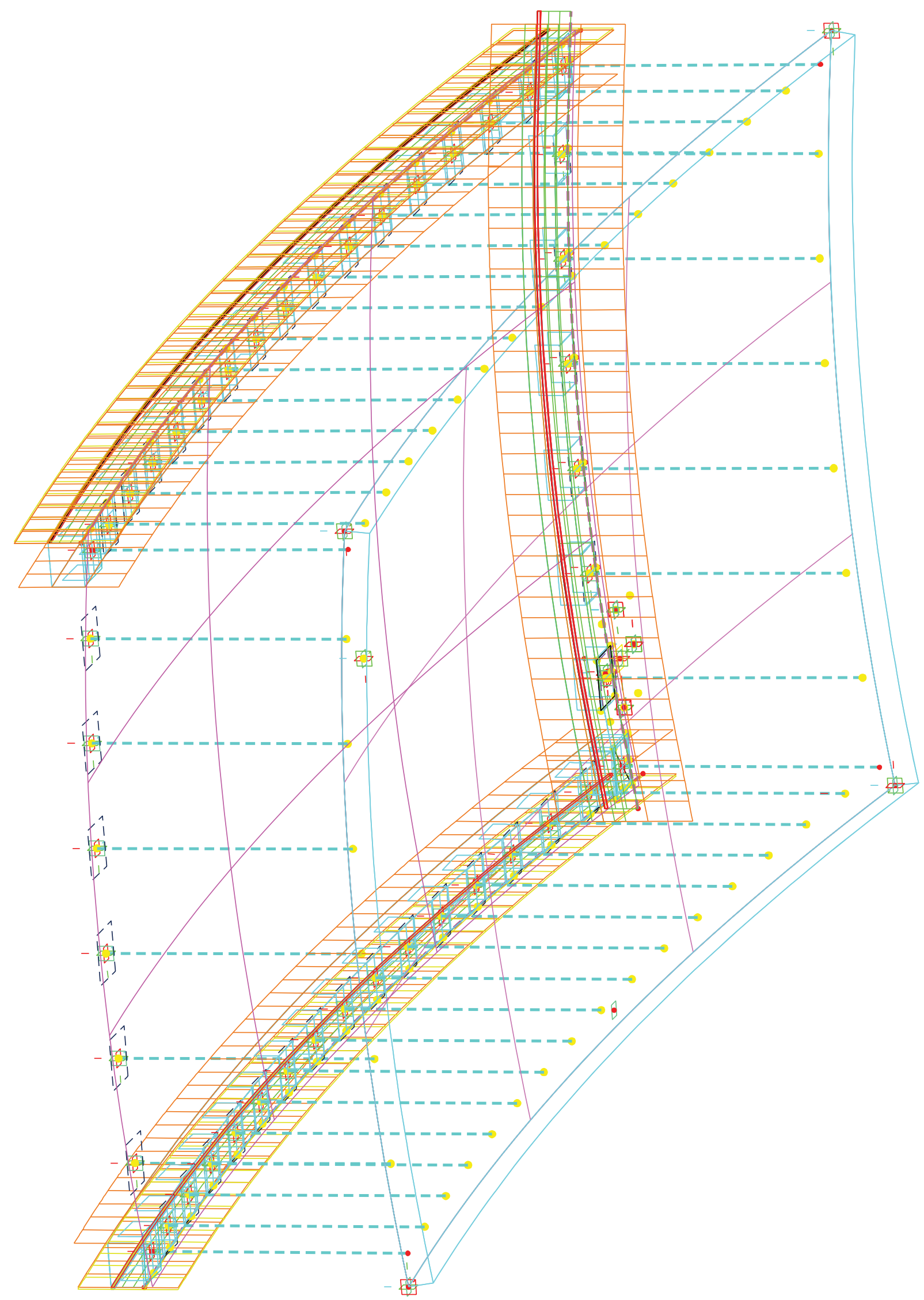

Additional view of Entablature modelling stage development. 


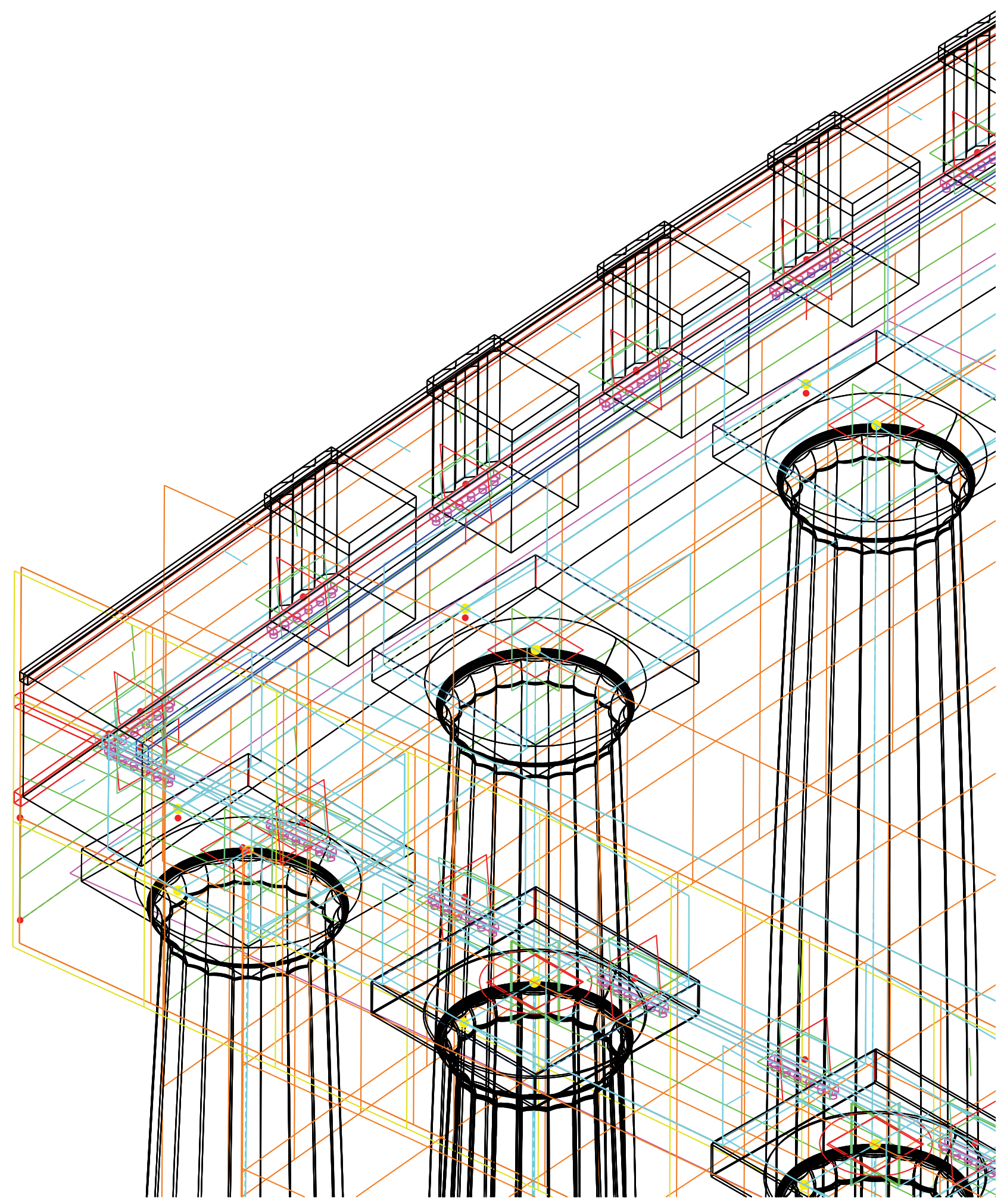

Enlargement of Figure 25; Epistylion modelling stage 


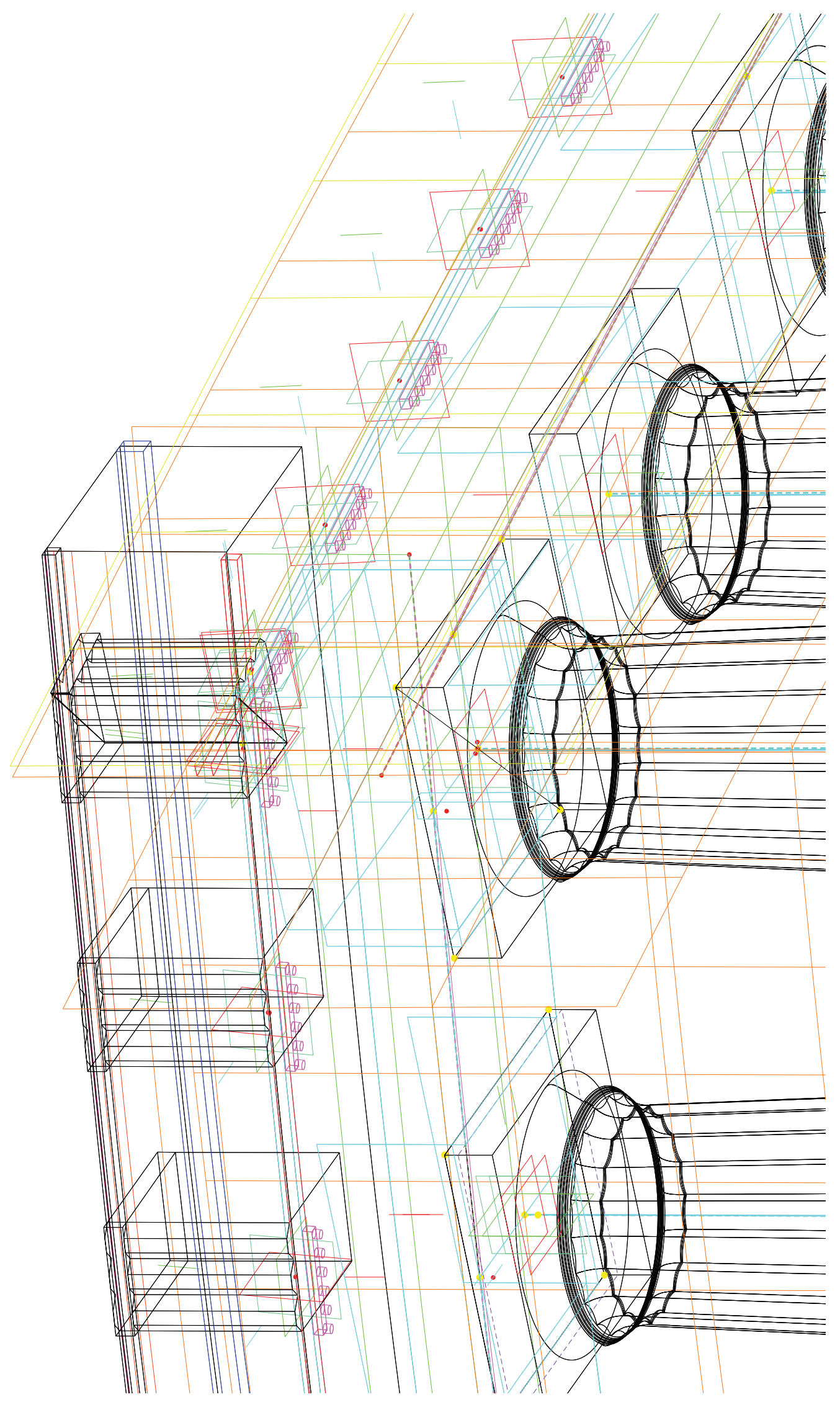

Enlargement of Figure 25; Epistylion modelling stage 


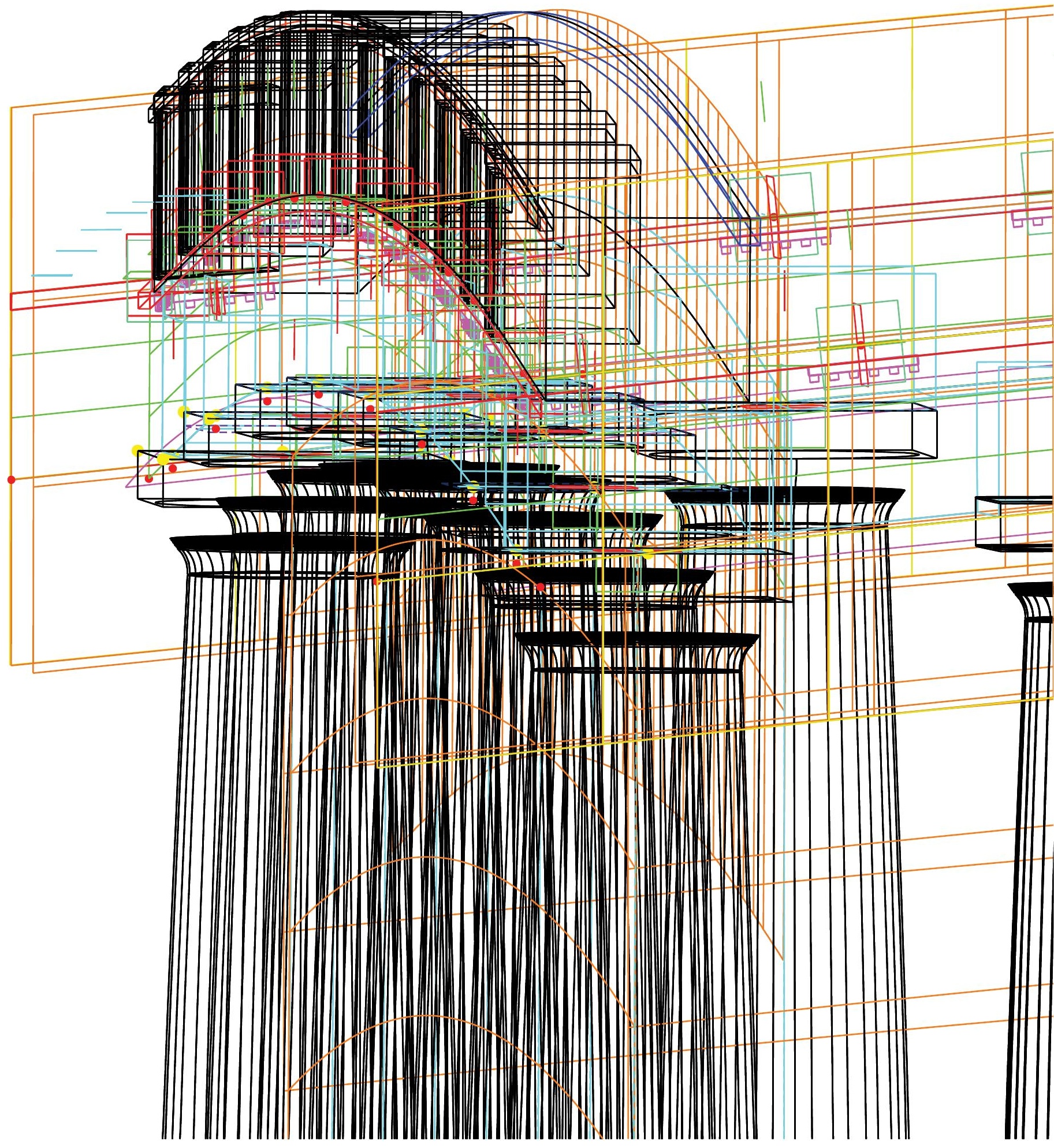

Additional view of Entablature modelling stage development. 


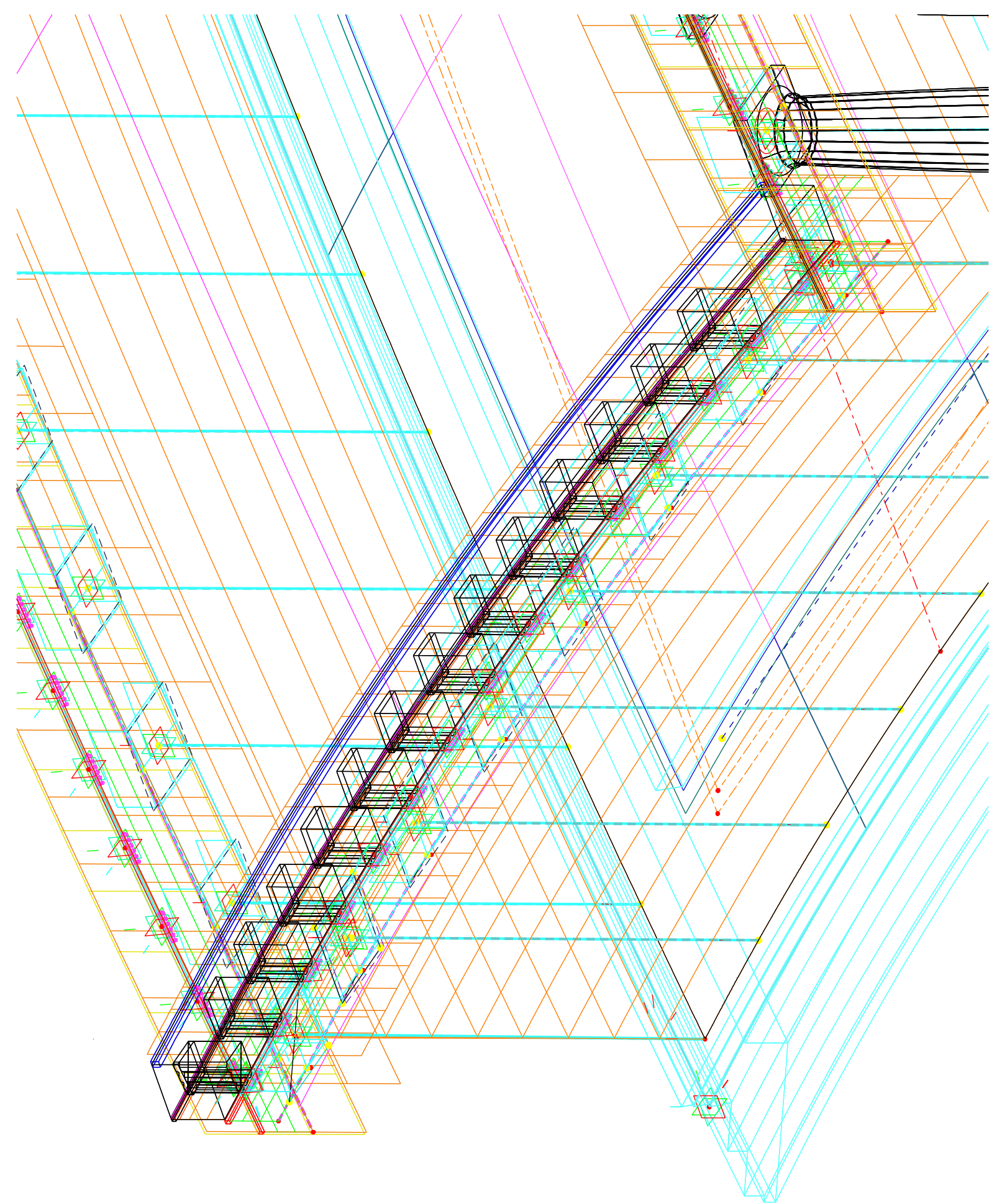

Enlargement of Figure 25; Epistylion modelling stage 


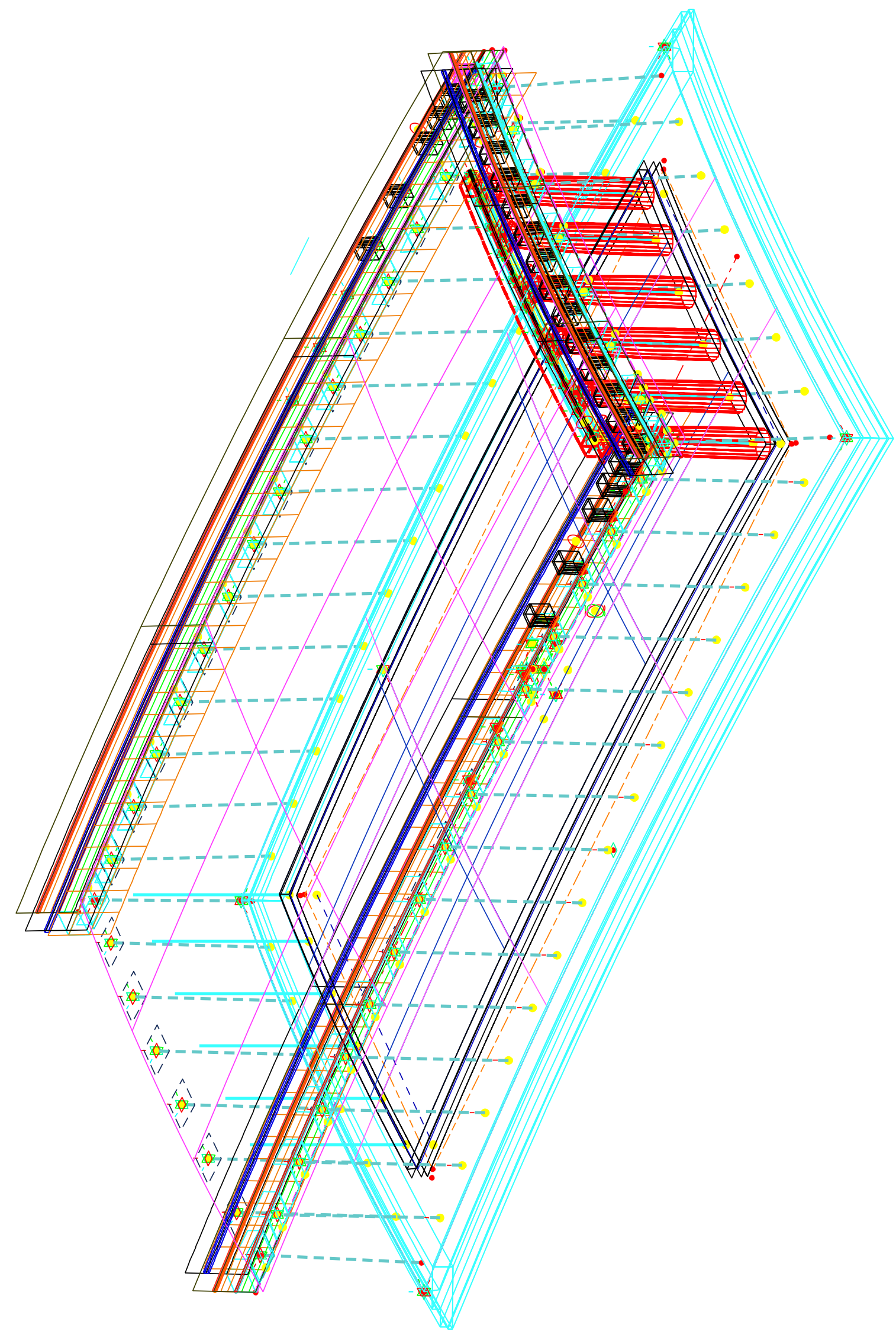

Additional view of Entablature modelling stage development. 


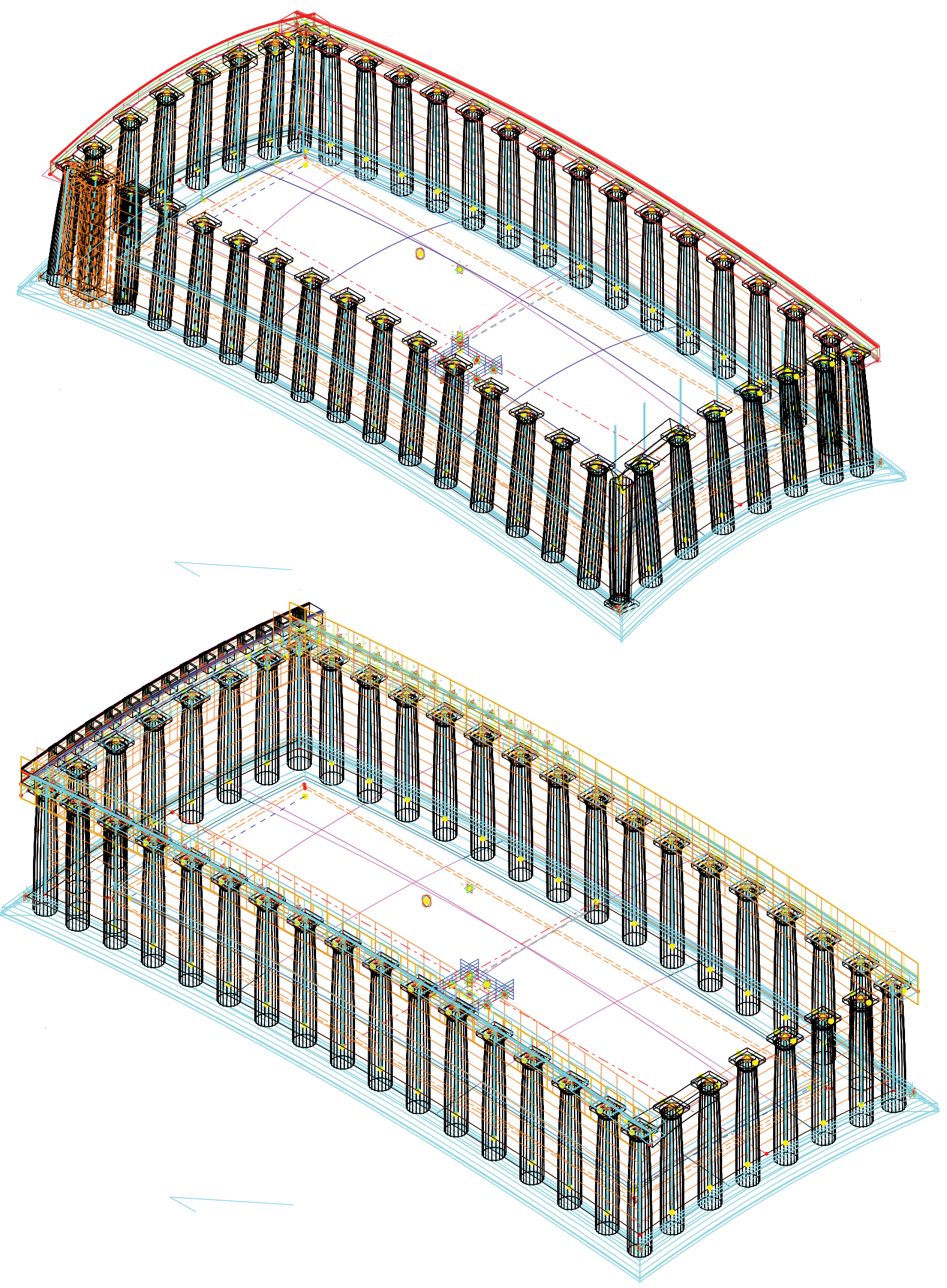

${ }^{29}$ Enlargement of Figure 26; Integrated model workarounds causing errors 


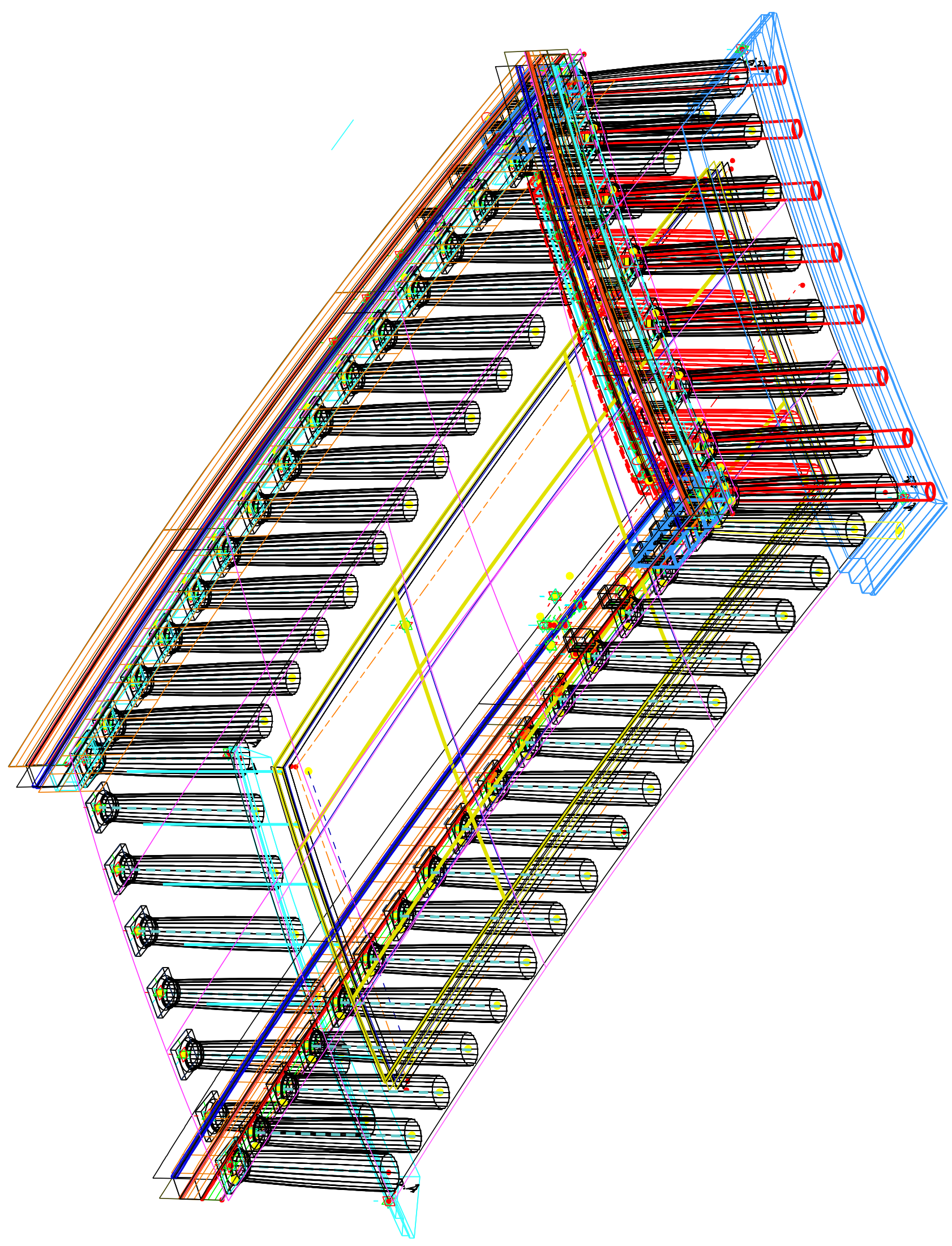

Additional view of final model with control rigs 


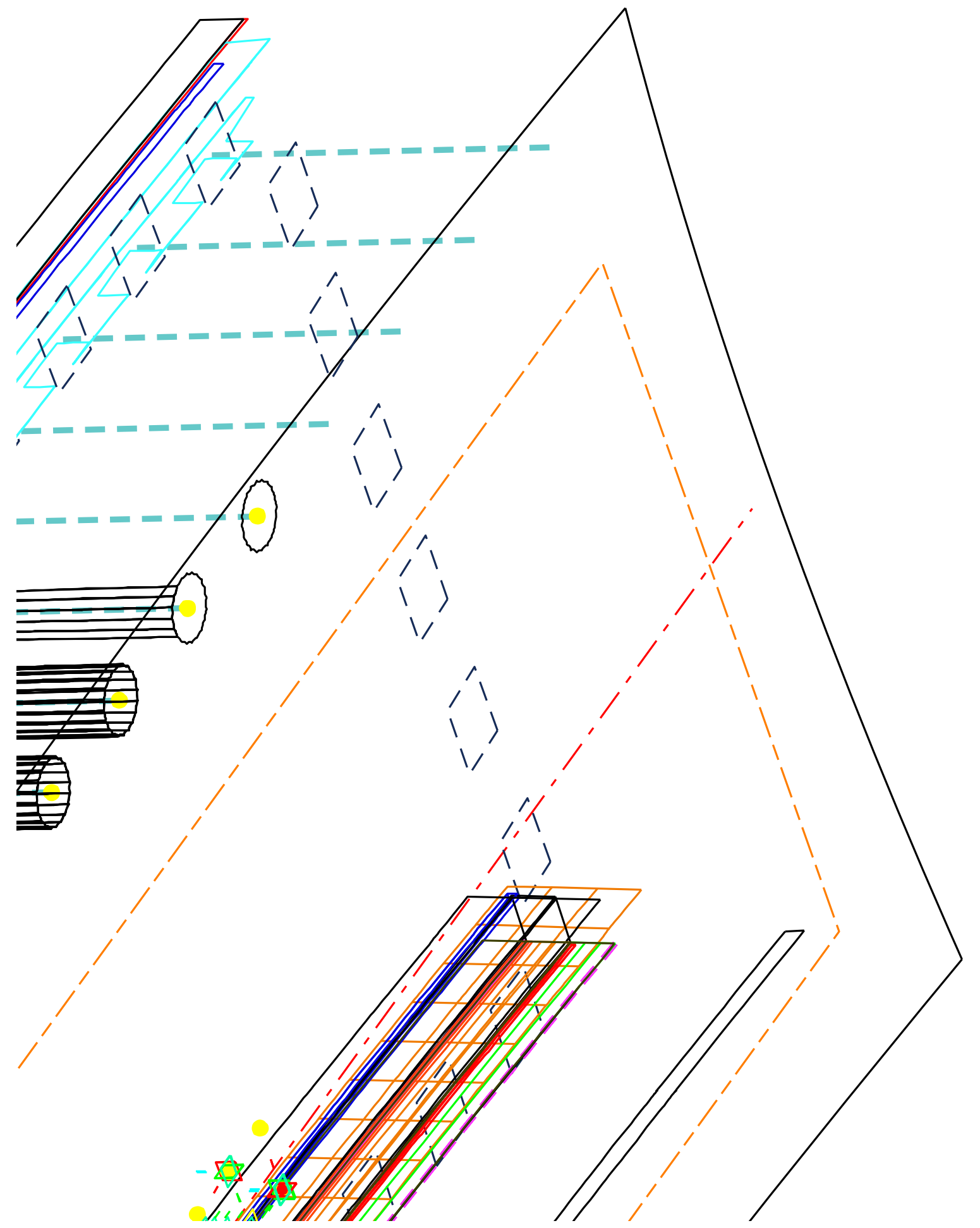

Additional view of final model with control rigs 


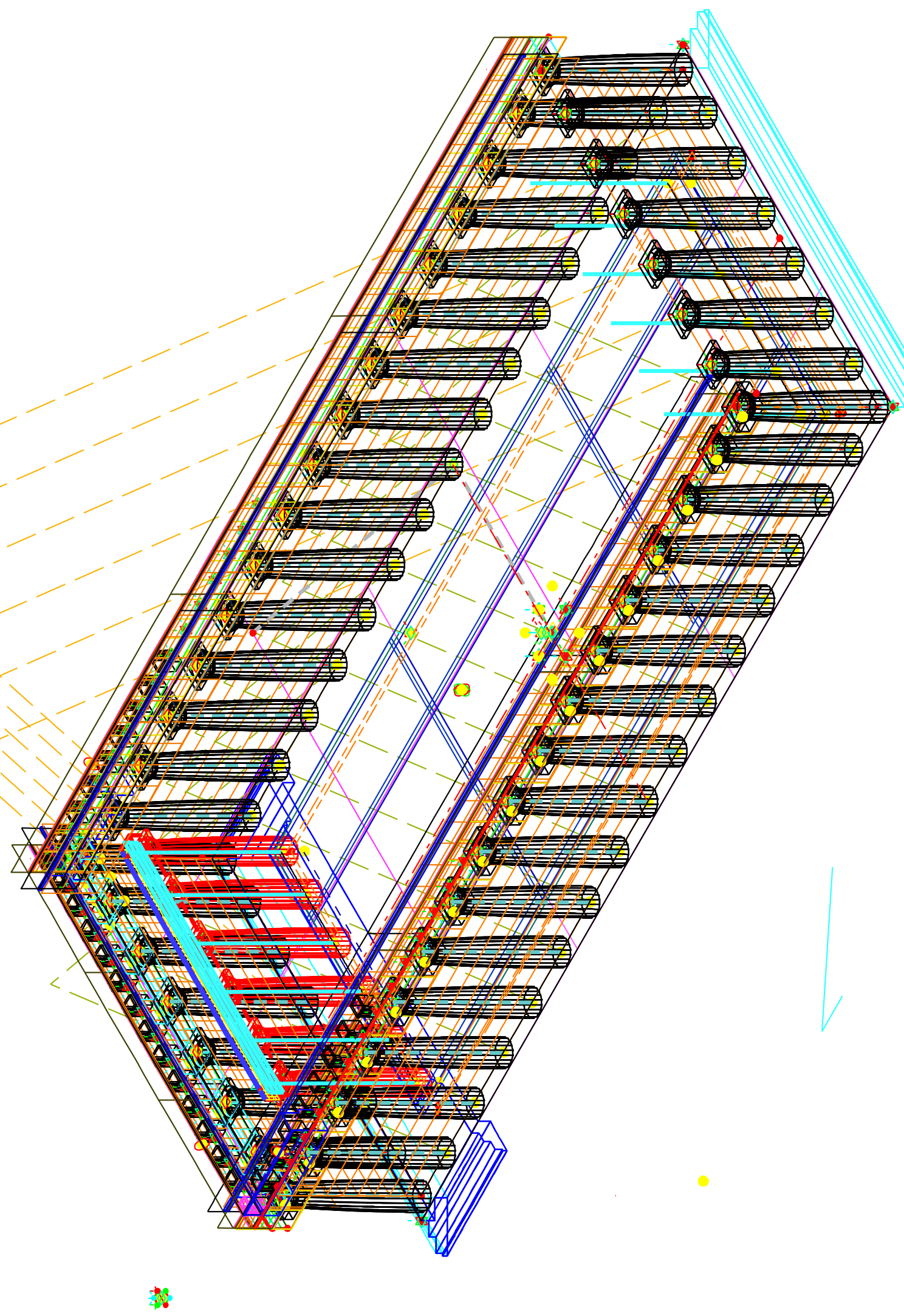

Enlargement of Figure 27; Final model with control rigs 


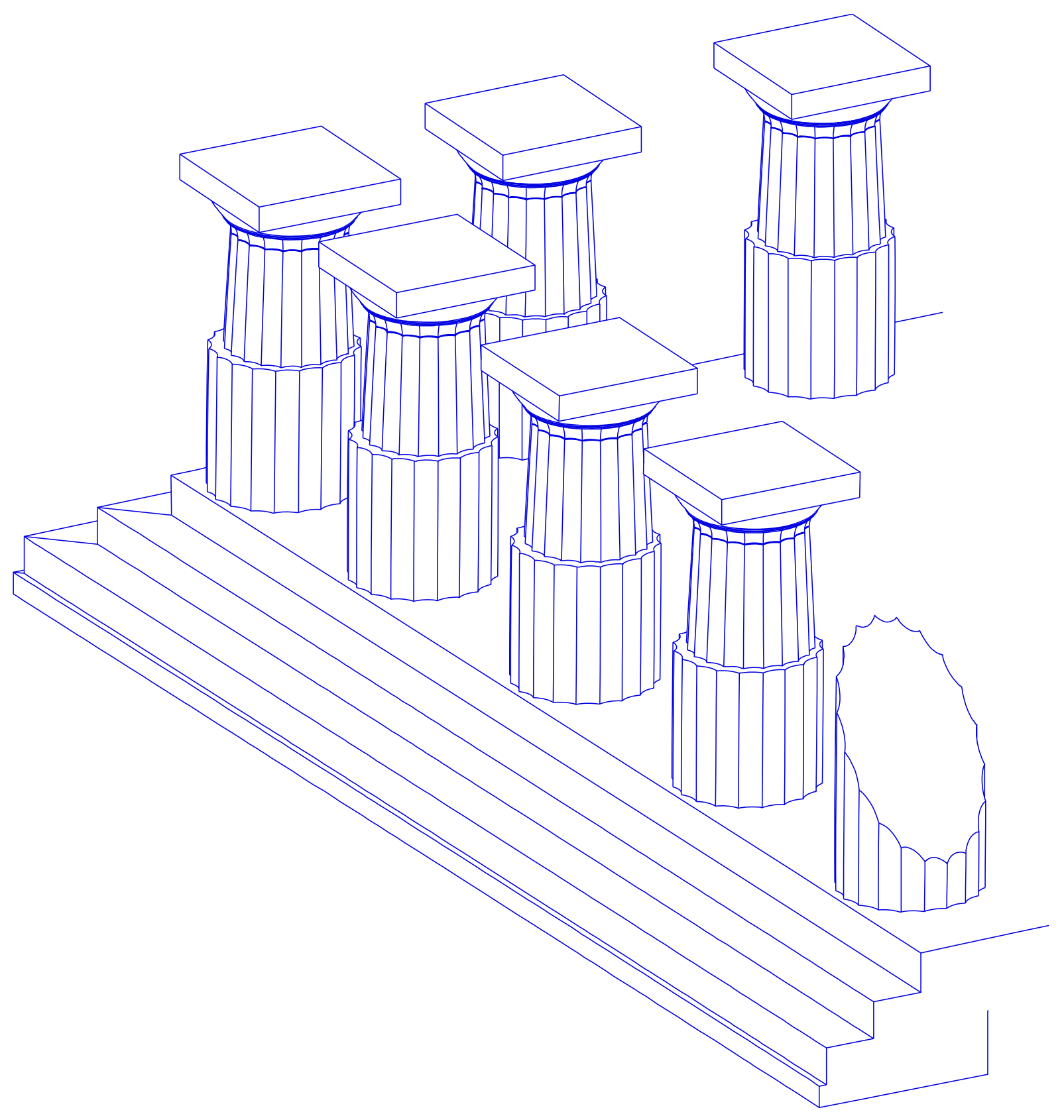

Additional view of final model 'solids' test 


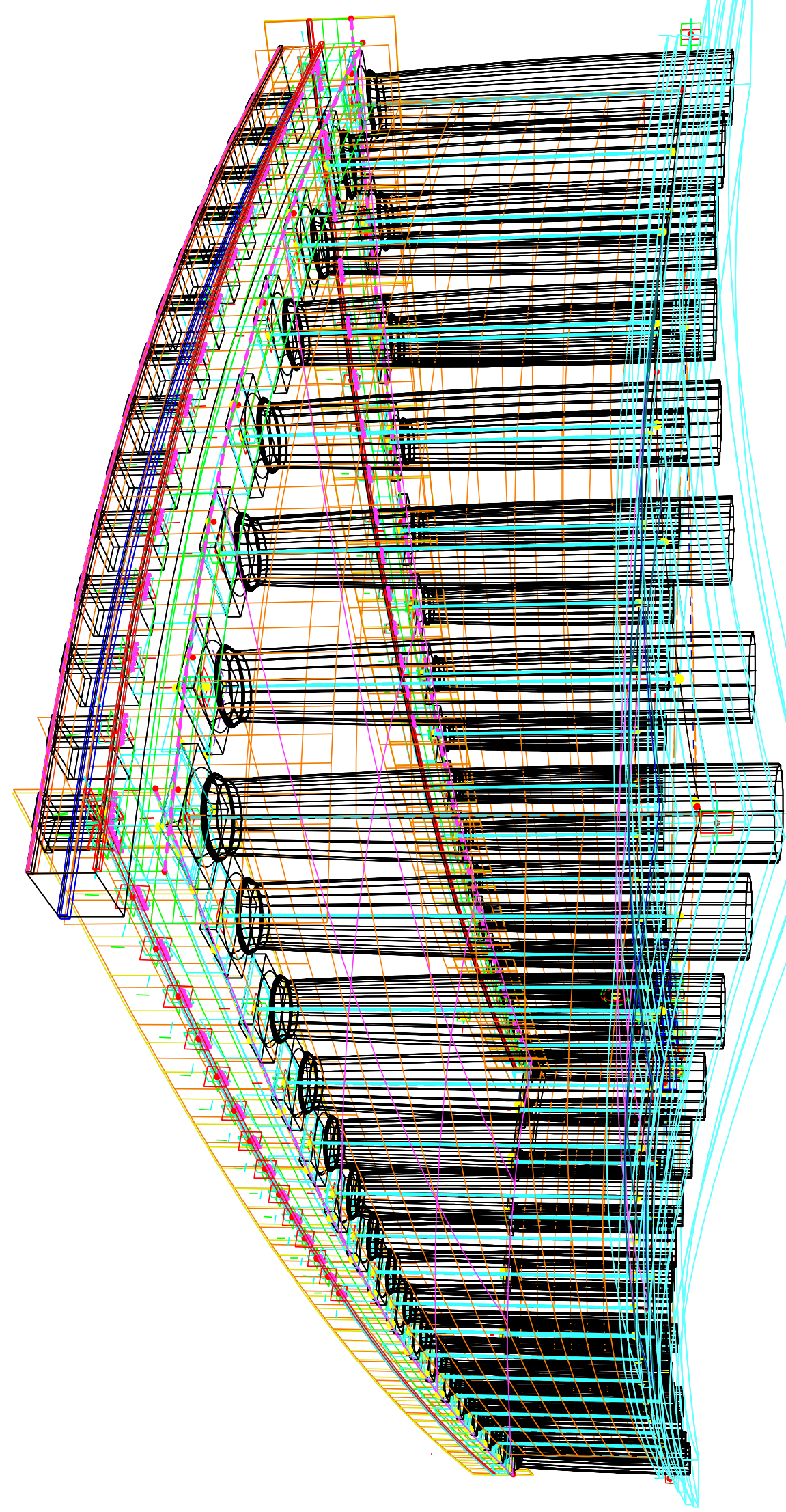

Additional view of final model with control rigs 


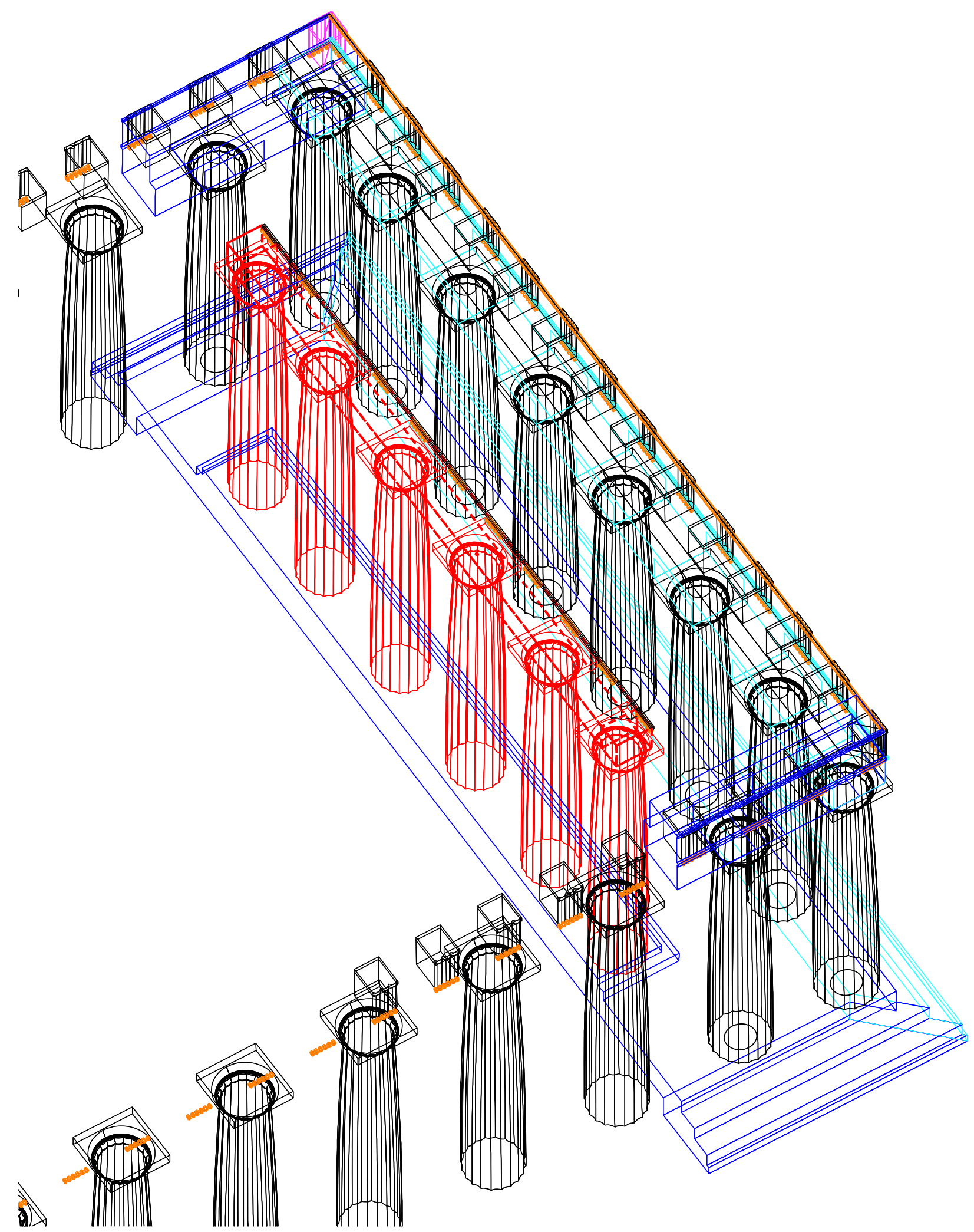

Enlargement of Figure 27; Final model without control rigs 

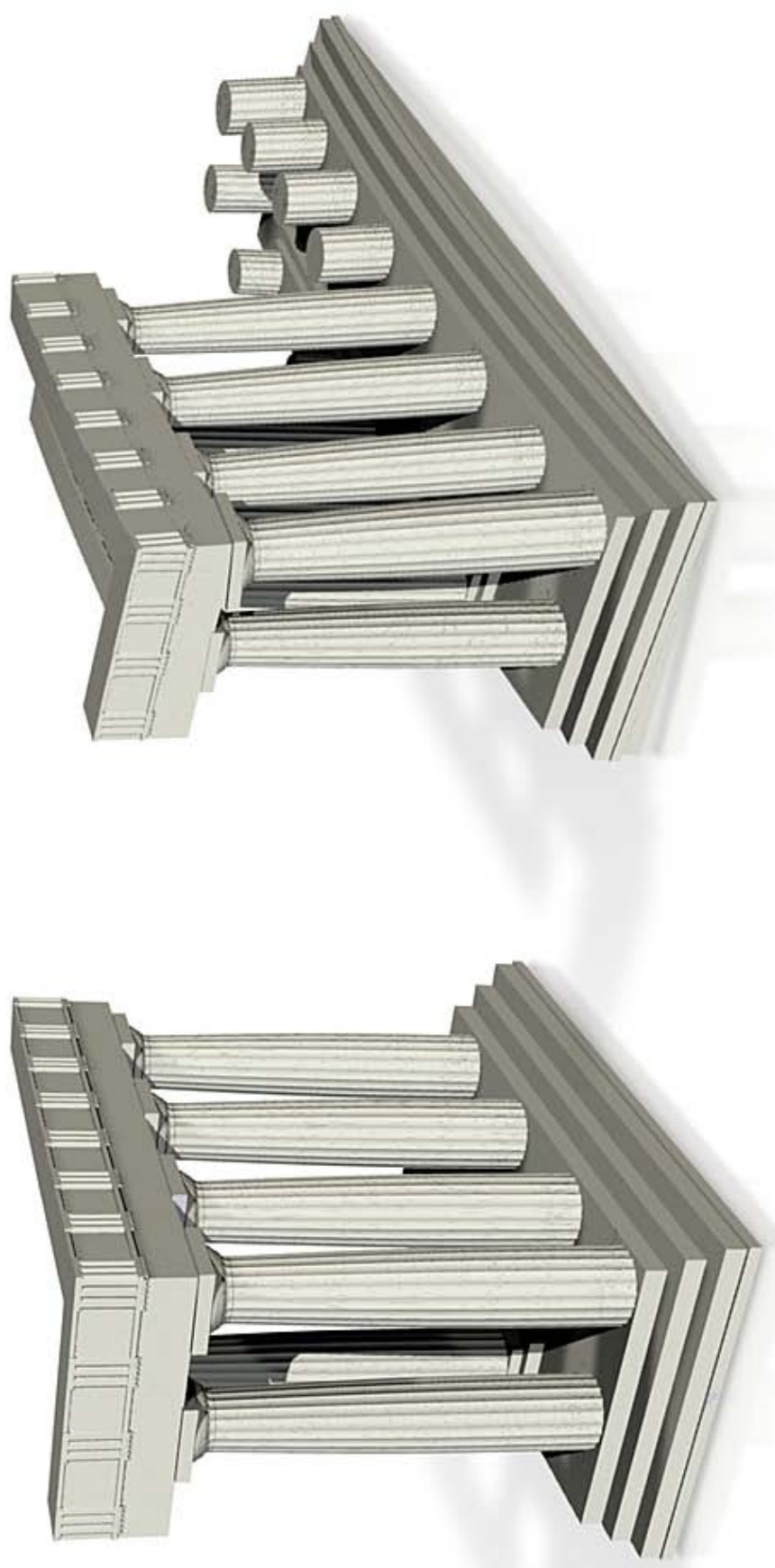

Additional rendered view of extracted final model instances 

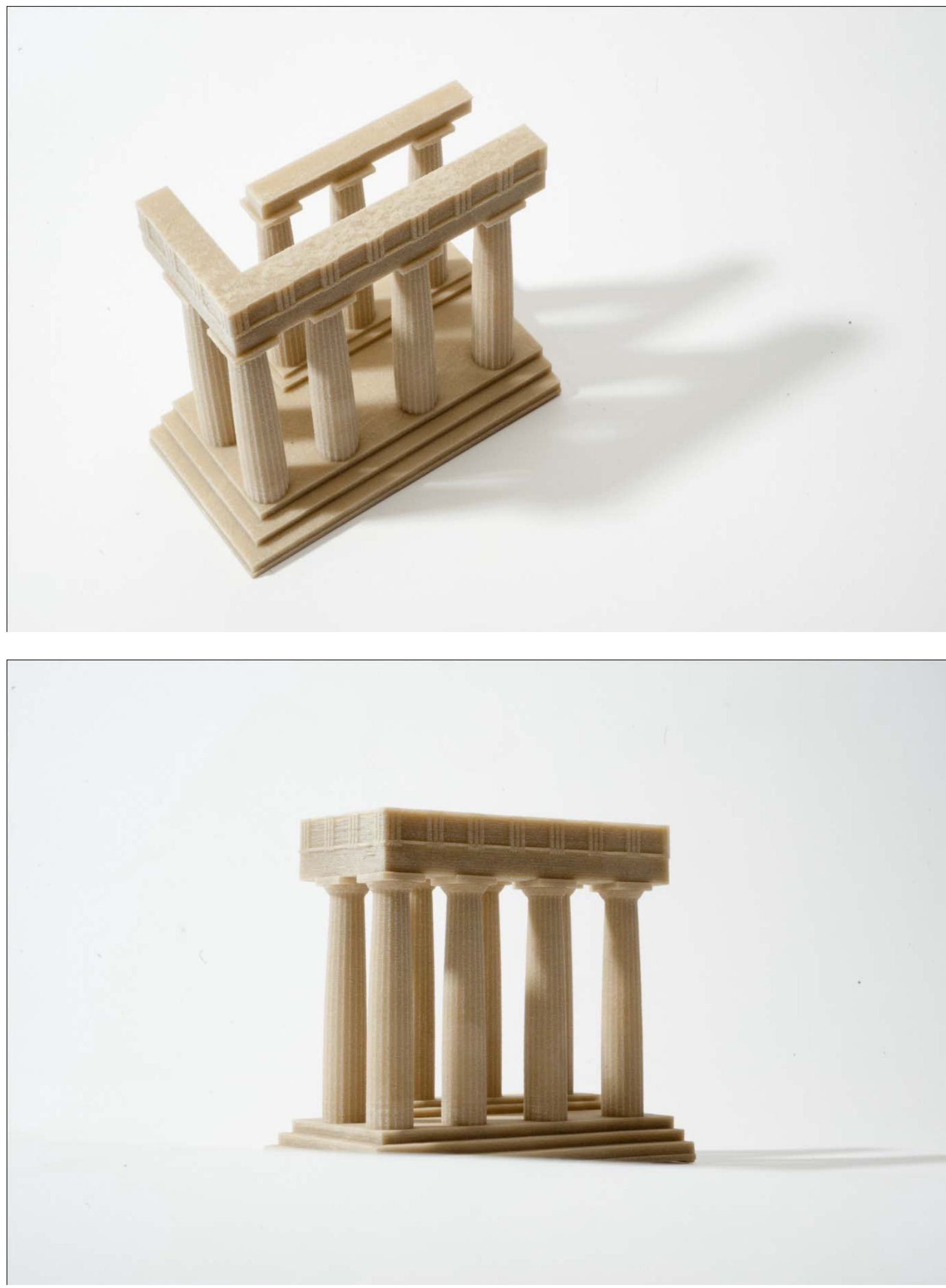

Final 1:150 Fused Layer Deposition models (images follow) 


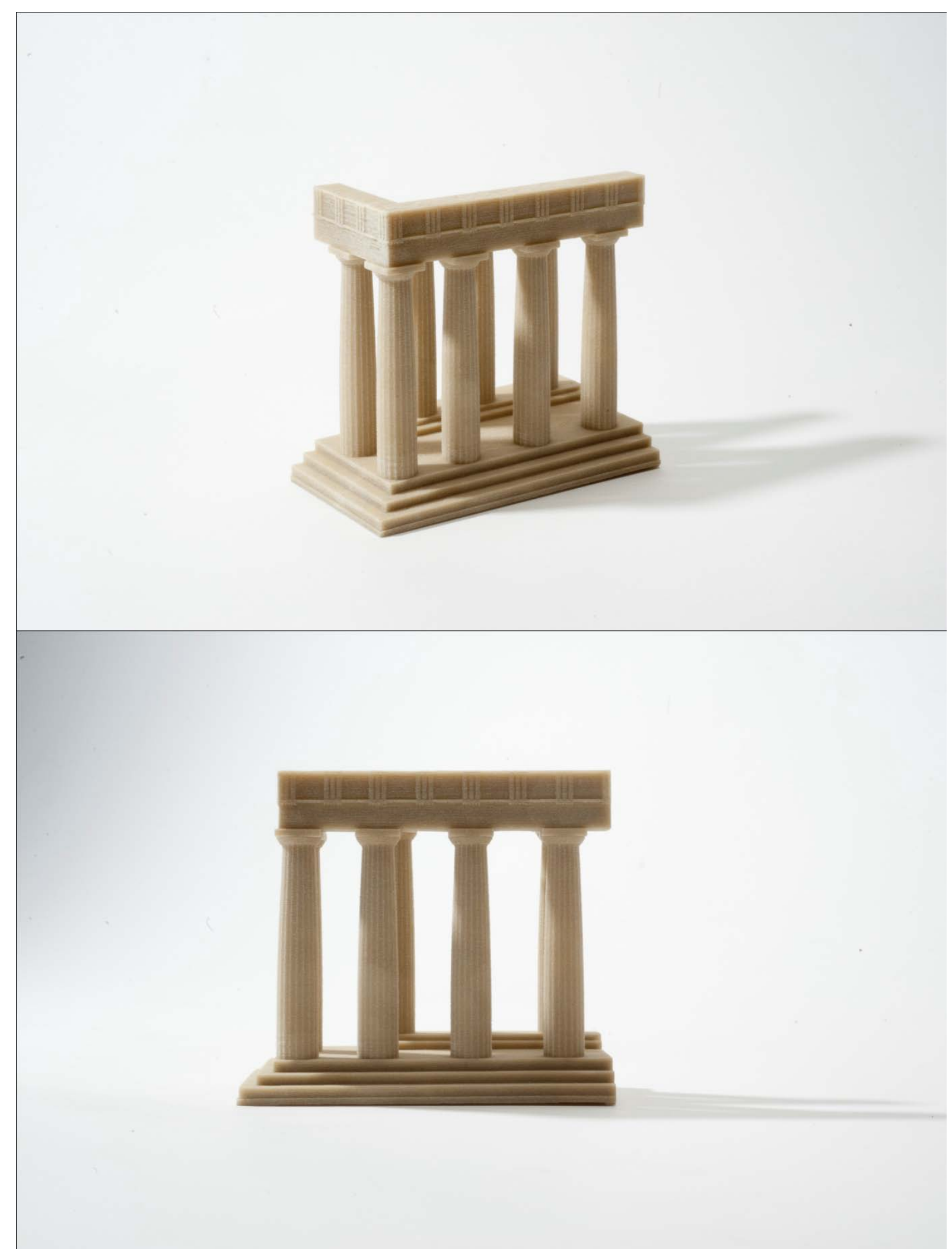




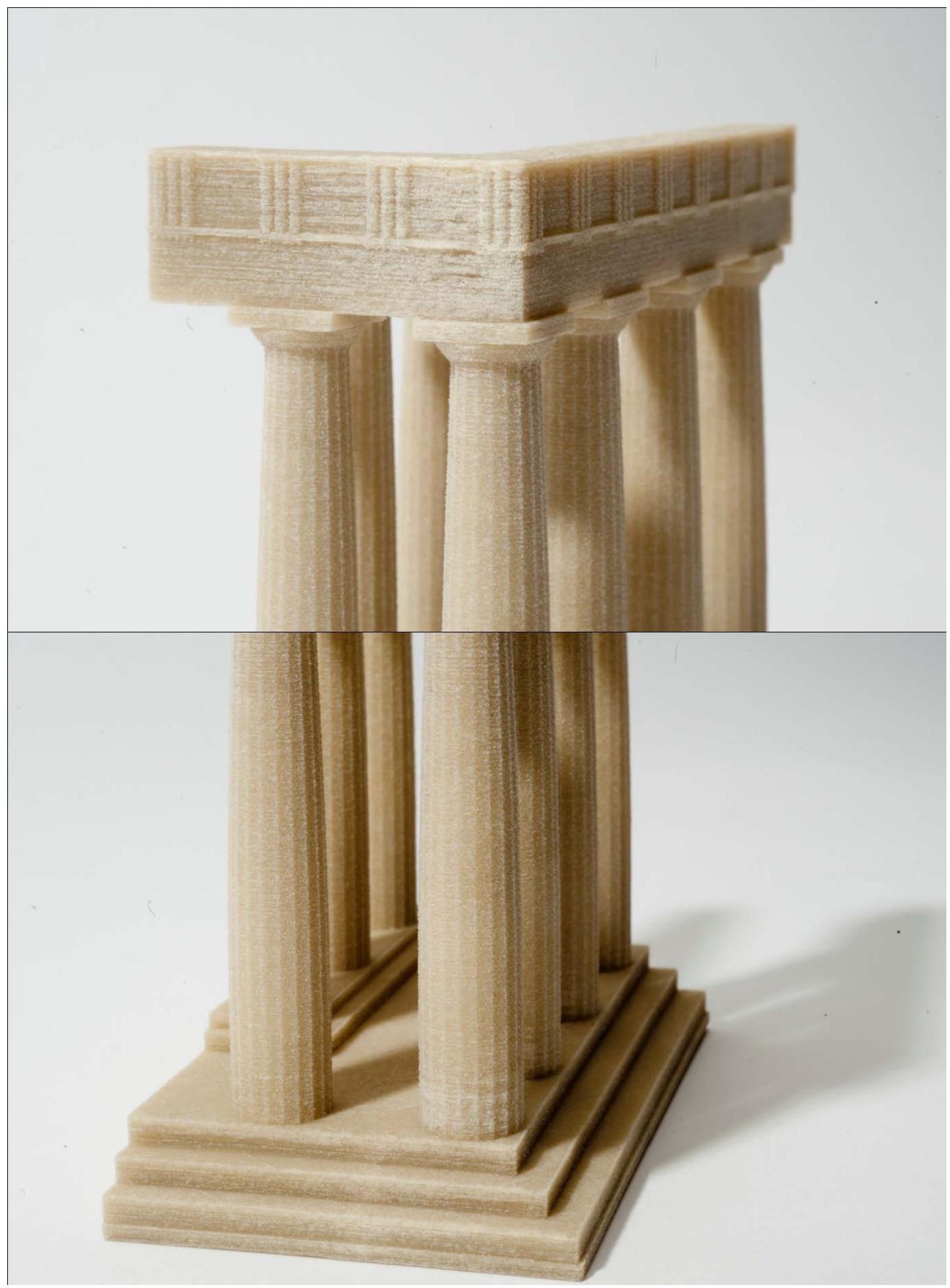




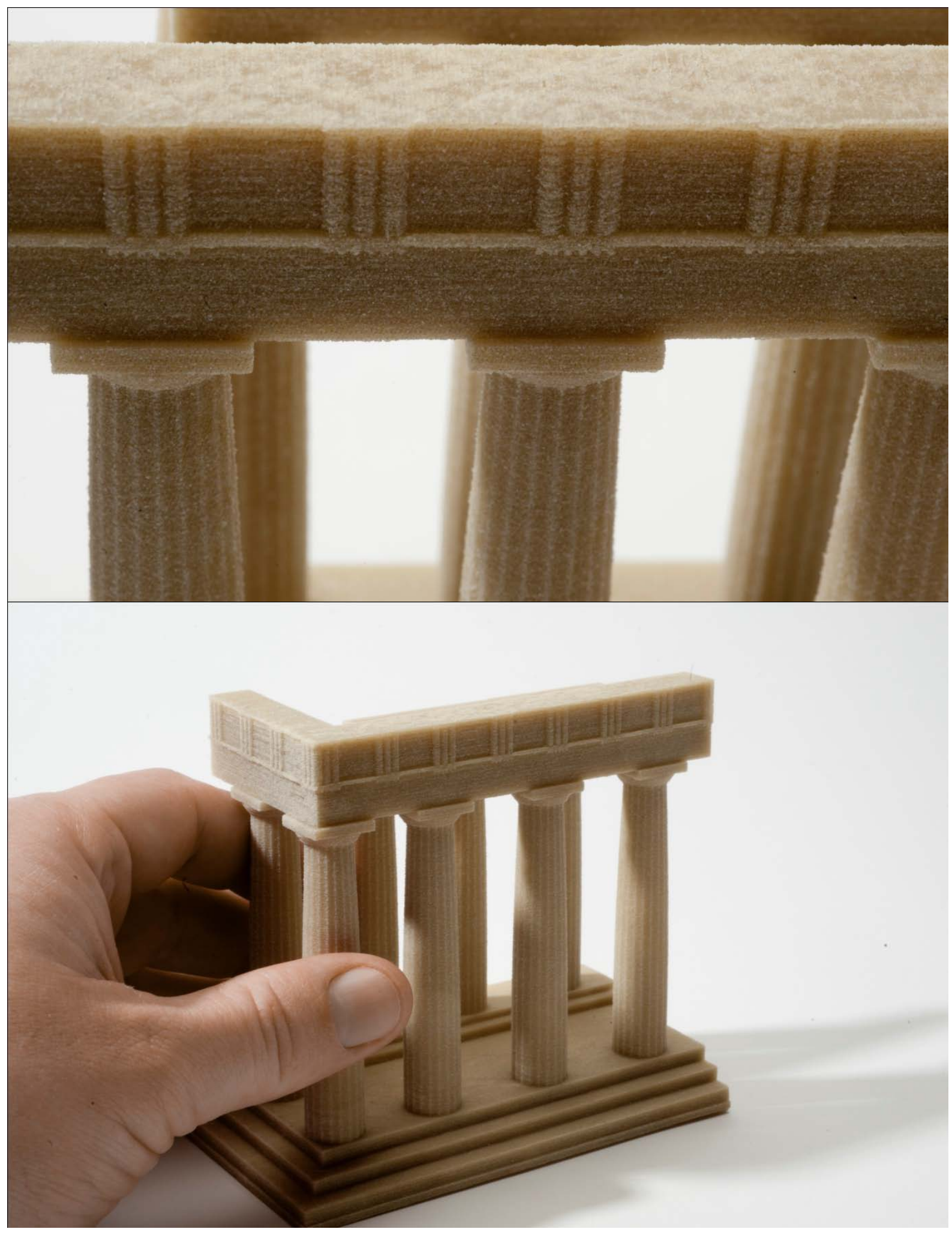




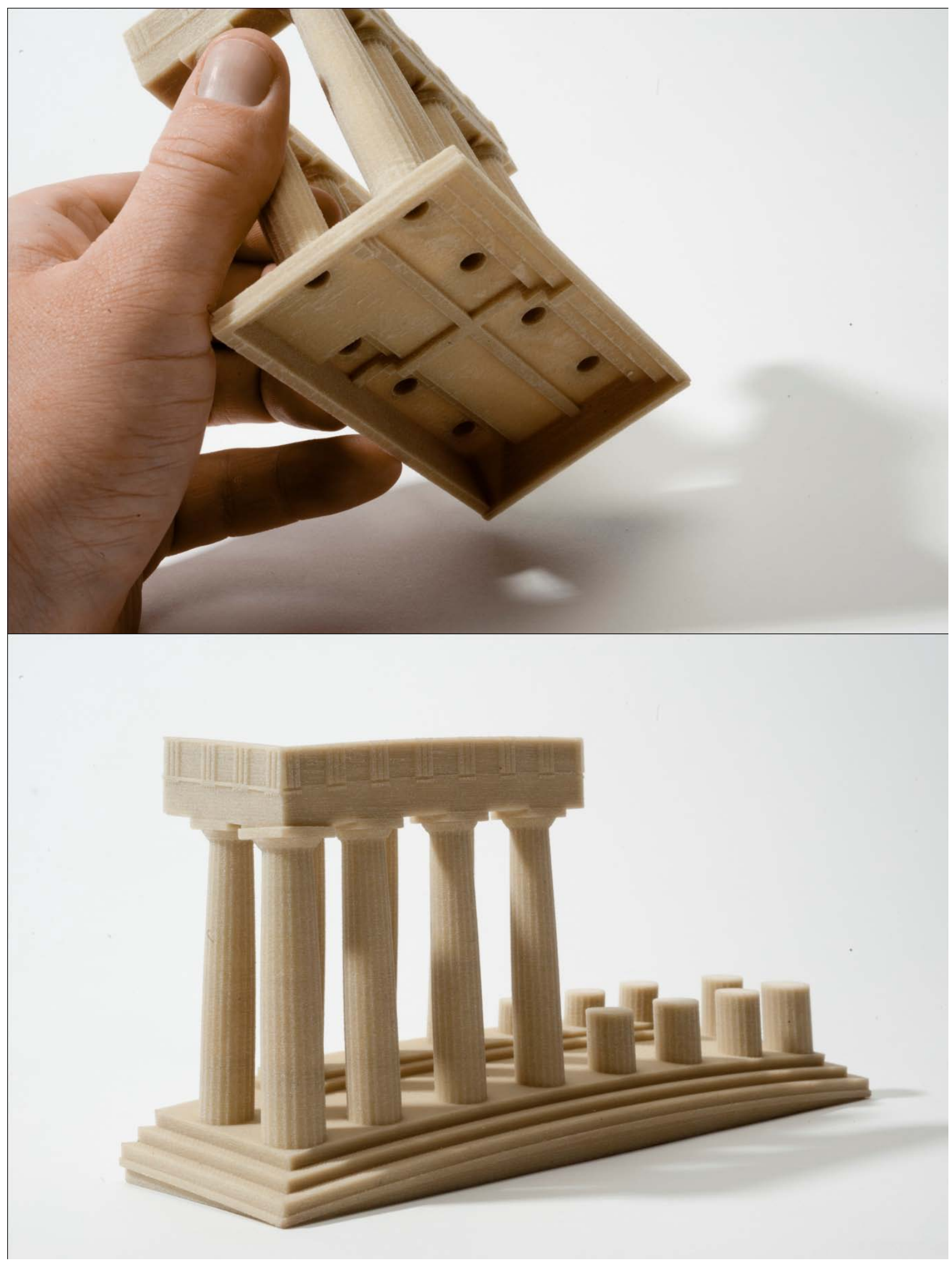




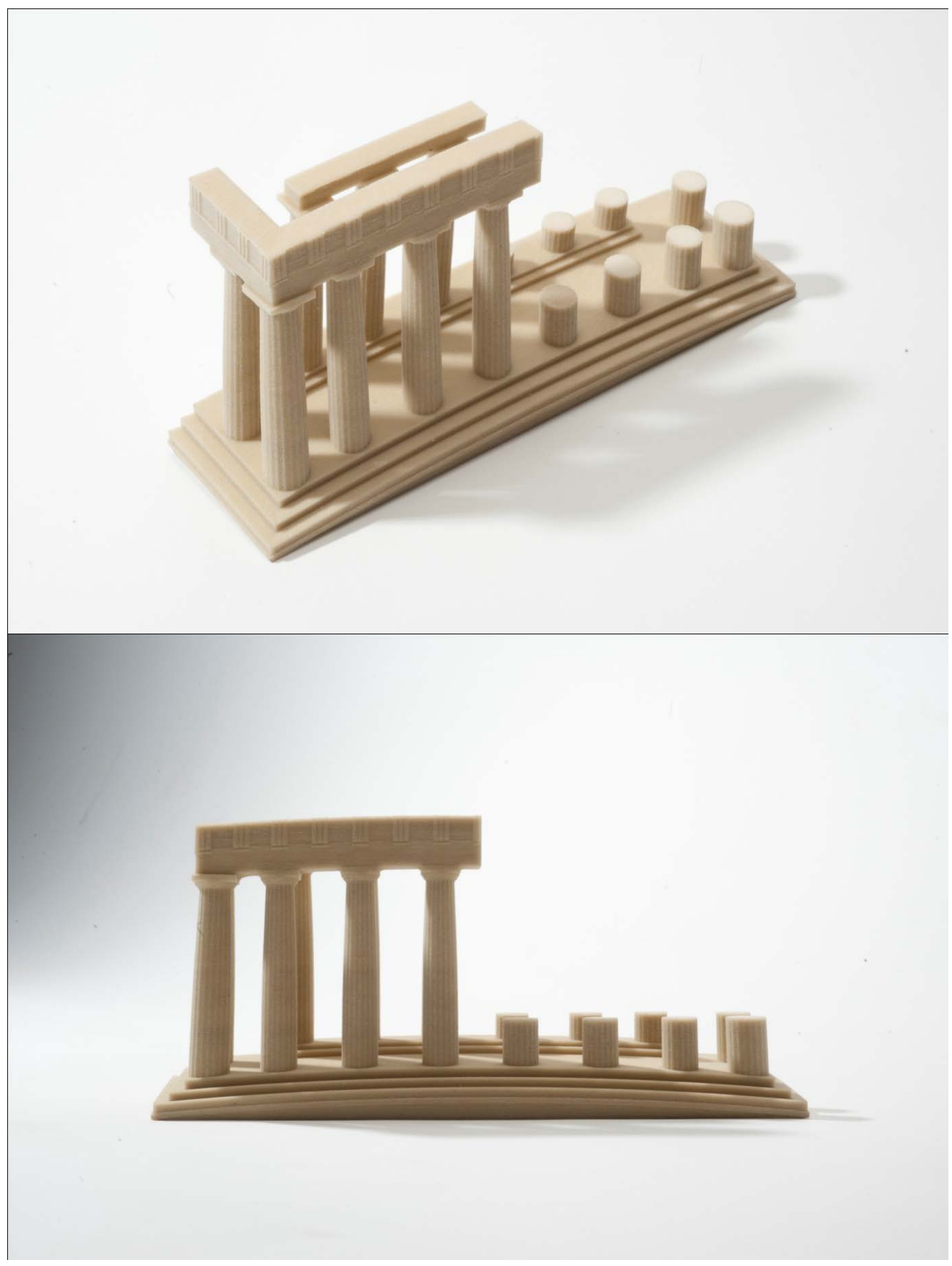




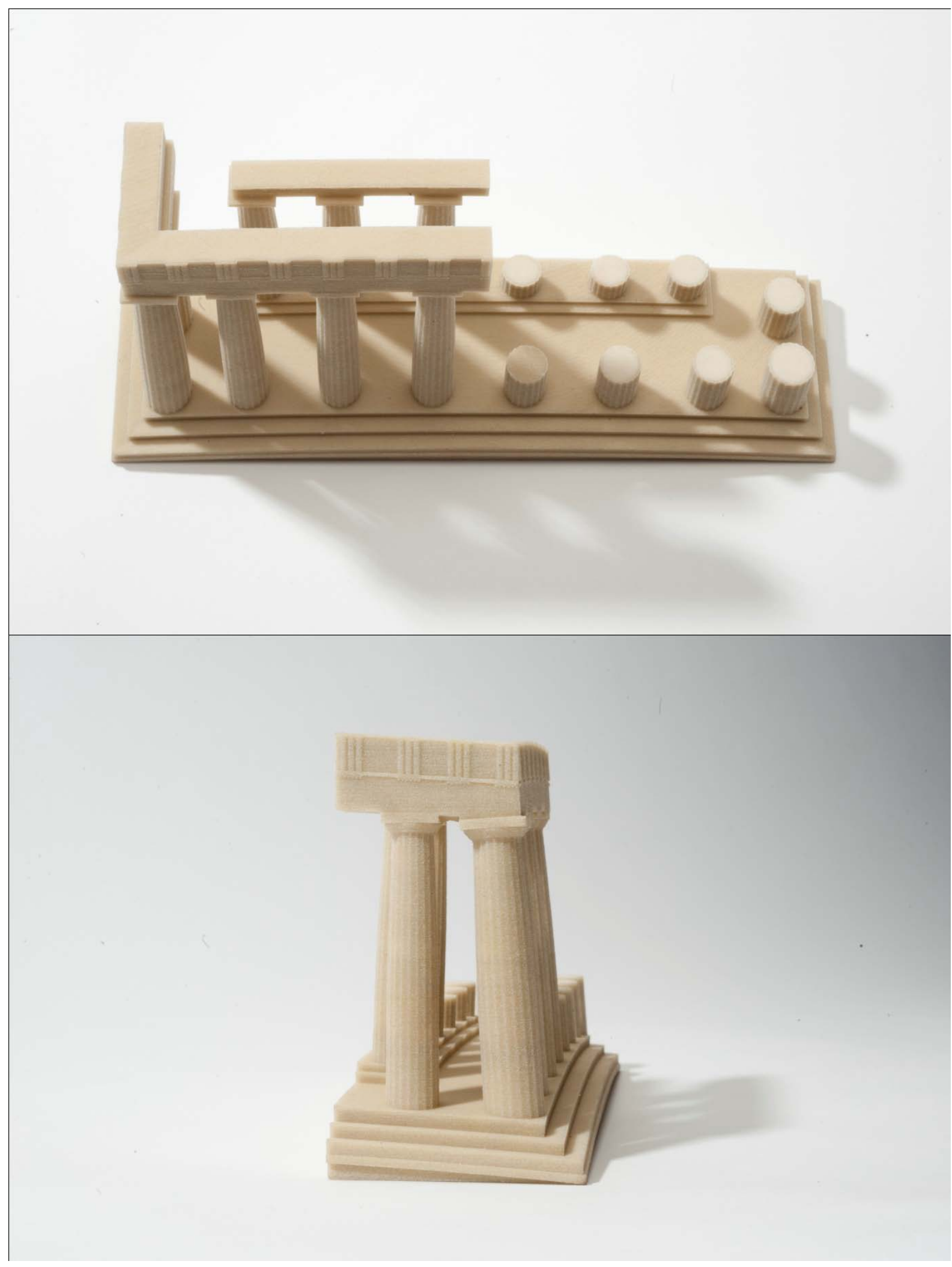




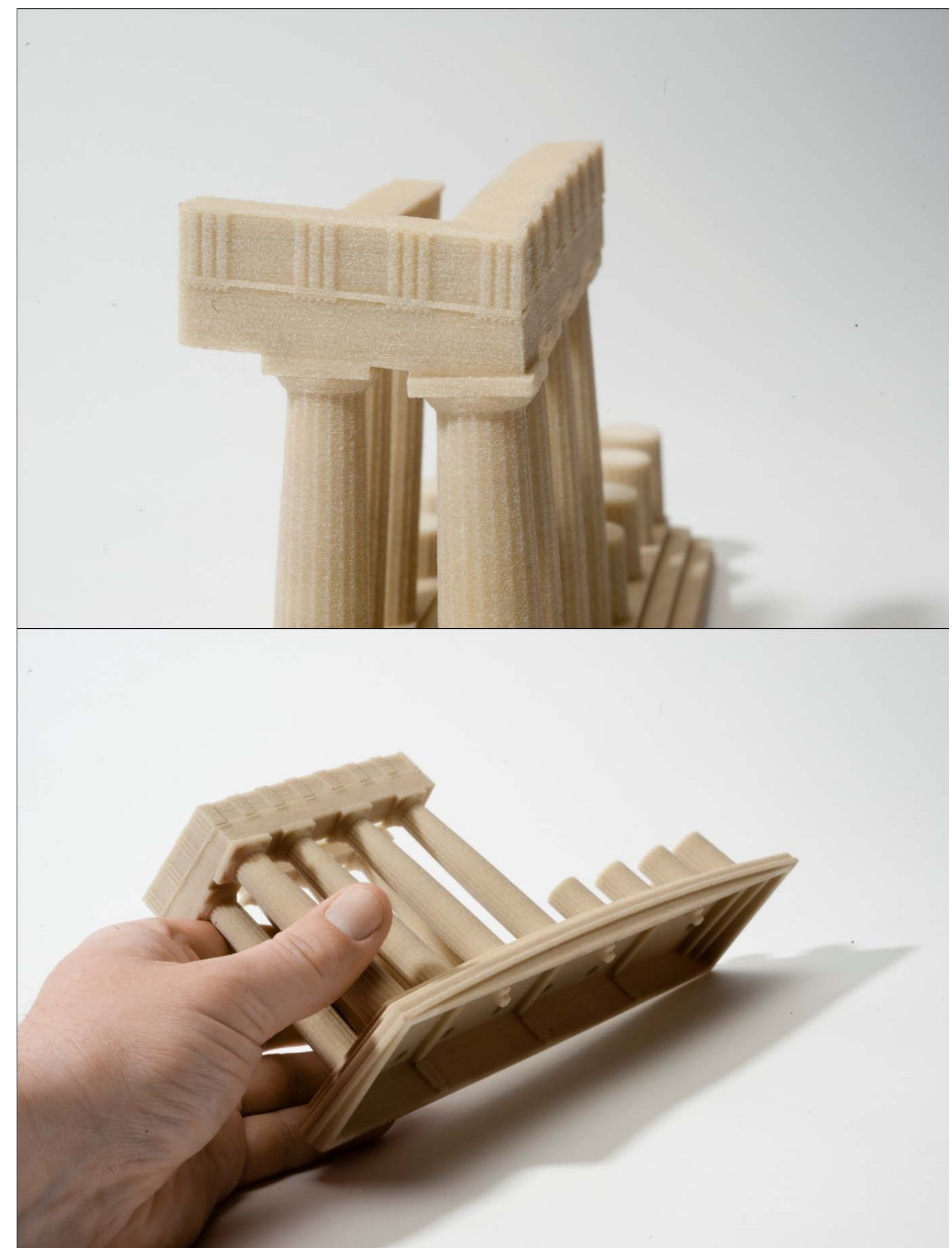




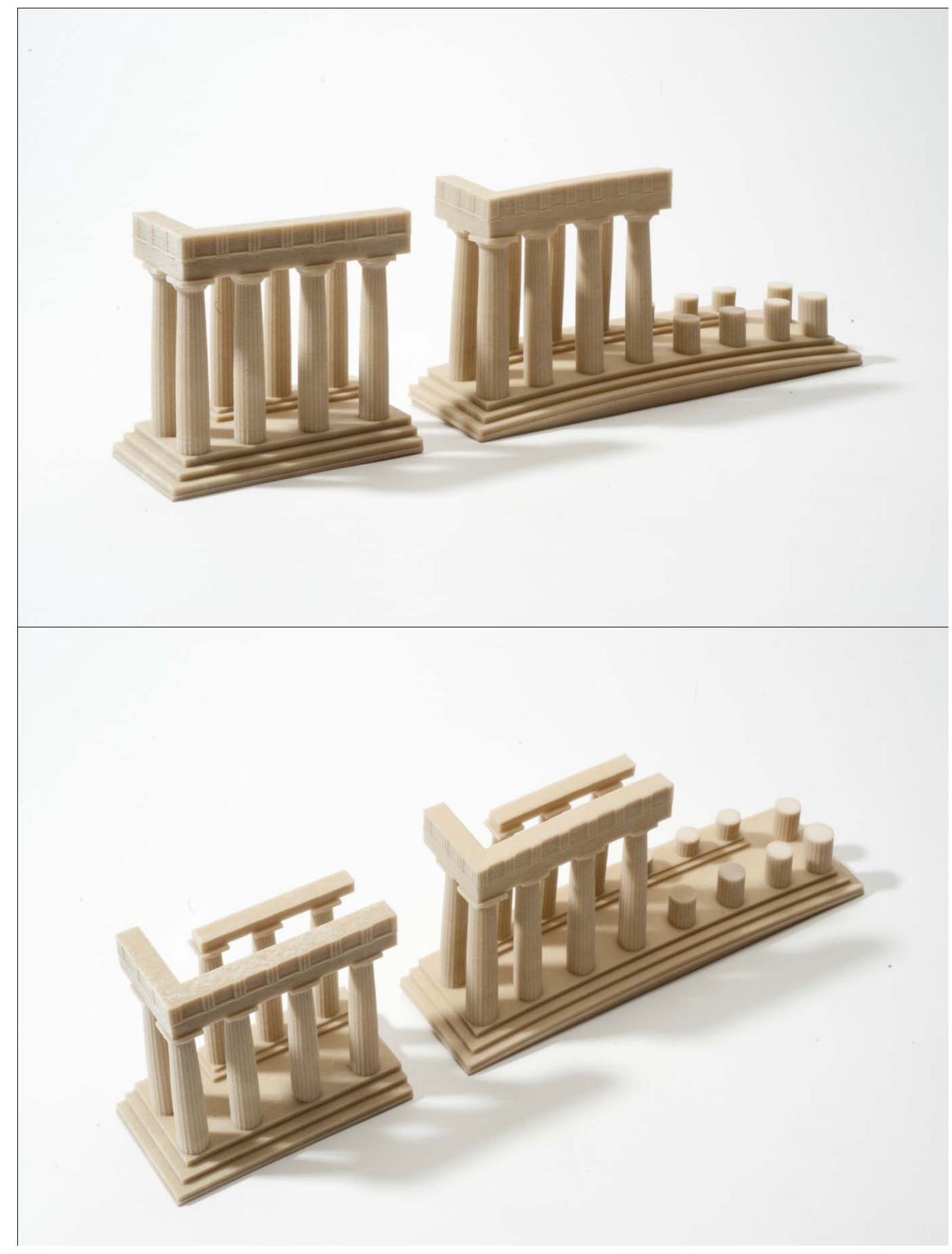




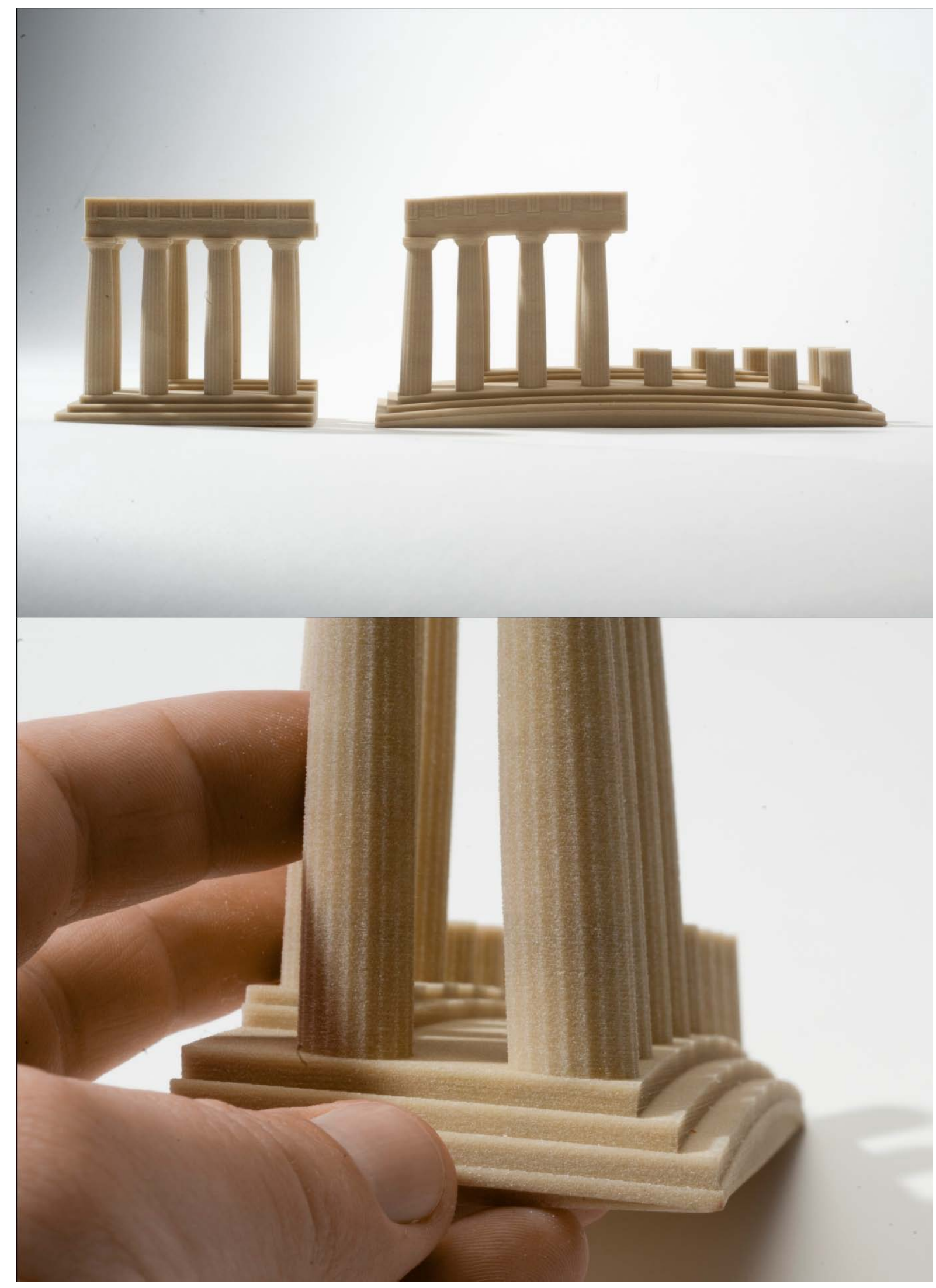




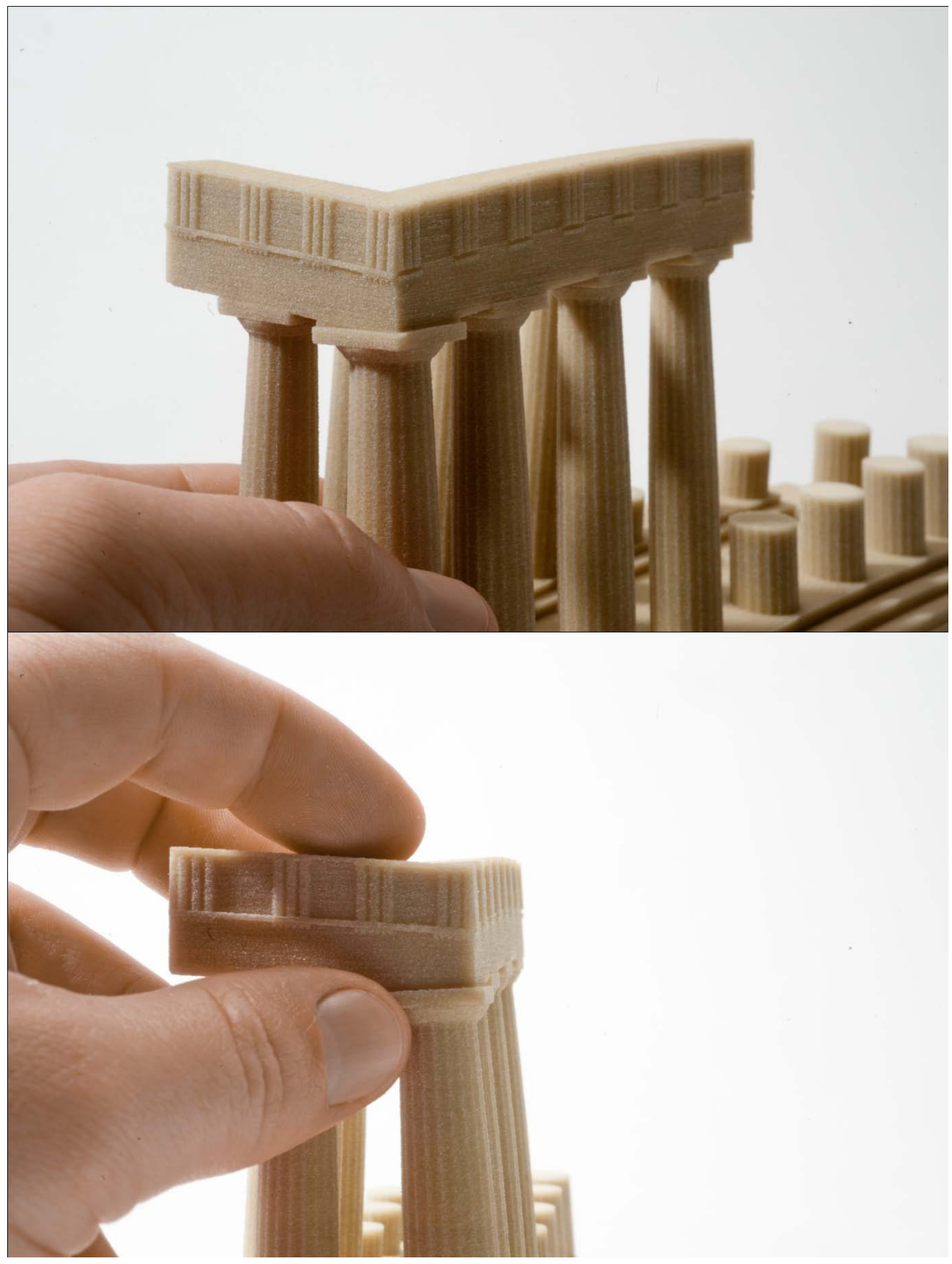




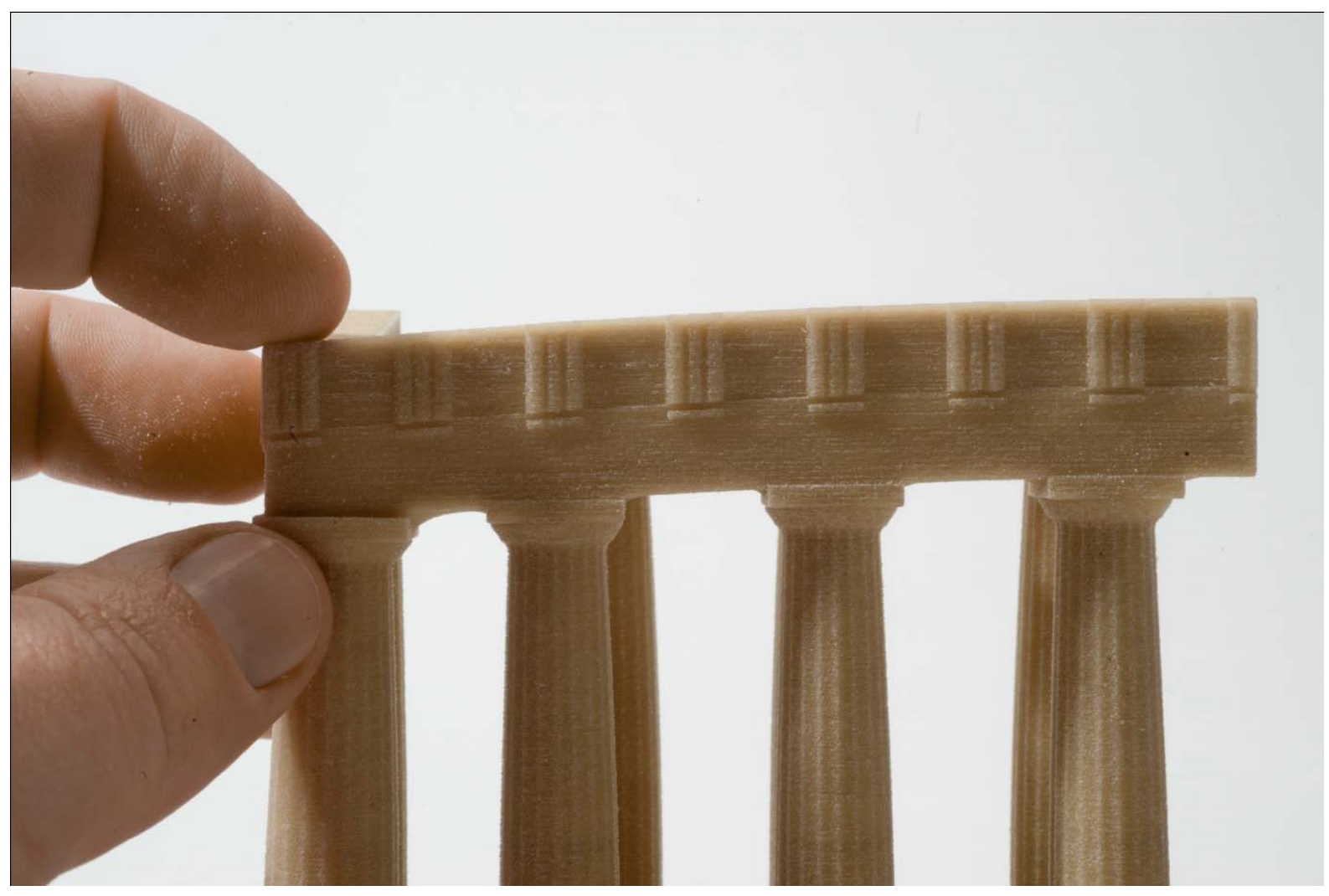

